\title{
Colorectal Cancer: From the Genetic Model to Posttranscriptional Regulation by Noncoding RNAs
}

\author{
María Antonia Lizarbe, ${ }^{1}$ Jorge Calle-Espinosa, ${ }^{1}$ Eva Fernández-Lizarbe, ${ }^{2}$ \\ Sara Fernández-Lizarbe, ${ }^{1}$ Miguel Ángel Robles, ${ }^{1}$ Nieves Olmo, ${ }^{1}$ and Javier Turnay ${ }^{1}$ \\ ${ }^{1}$ Departamento de Bioquímica y Biología Molecular I, Facultad de Ciencias Químicas, Universidad Complutense, \\ 28040 Madrid, Spain \\ ${ }^{2}$ Departamento de Oncología Radioterápica, Hospital Universitario Ramón y Cajal, 28034 Madrid, Spain \\ Correspondence should be addressed to Javier Turnay; turnay@ucm.es
}

Received 5 December 2016; Accepted 16 February 2017; Published 10 May 2017

Academic Editor: Michael Linnebacher

Copyright (C) 2017 María Antonia Lizarbe et al. This is an open access article distributed under the Creative Commons Attribution License, which permits unrestricted use, distribution, and reproduction in any medium, provided the original work is properly cited.

Colorectal cancer is the third most common form of cancer in developed countries and, despite the improvements achieved in its treatment options, remains as one of the main causes of cancer-related death. In this review, we first focus on colorectal carcinogenesis and on the genetic and epigenetic alterations involved. In addition, noncoding RNAs have been shown to be important regulators of gene expression. We present a general overview of what is known about these molecules and their role and dysregulation in cancer, with a special focus on the biogenesis, characteristics, and function of microRNAs. These molecules are important regulators of carcinogenesis, progression, invasion, angiogenesis, and metastases in cancer, including colorectal cancer. For this reason, miRNAs can be used as potential biomarkers for diagnosis, prognosis, and efficacy of chemotherapeutic treatments, or even as therapeutic agents, or as targets by themselves. Thus, this review highlights the importance of miRNAs in the development, progression, diagnosis, and therapy of colorectal cancer and summarizes current therapeutic approaches for the treatment of colorectal cancer.

\section{Introduction}

Colorectal cancer (CRC) is a major global cause of morbidity and mortality in developed countries. CRC is a heterogeneous disease regarding tumor localization and genetic and racial differences, and multiple interactions with environmental factors, diet, and style of life influence its development. CRC risk factors include hereditary components (i.e., hereditary nonpolyposis colorectal cancer), predisposal to polyp formation, large bowel inflammatory diseases, obesity, high fat diet, alcoholism, smoking, and stress [1-5]. The use of biomarkers to predict the risk for CRC or the existence of early stages of the tumor could contribute to decreasing the development of the disease and allowing early intervention. In this review, we give an overview of colorectal carcinogenesis mechanisms and therapies that are currently being used. Among the multiple factors involved in CRC development and progression, in the last years, the noncoding
RNA molecules have been identified as important factors regulating many biological pathways. For this reason, we also focus on the regulatory effects of noncoding RNAs and their role in CRC development. Among these RNA molecules, microRNA (miRNAs) expression dysregulation has been reported as an important factor in the development of CRC. Moreover, they can also be considered as good biomarkers for the early detection of CRC in plasma or stool, as well as predictors of therapy efficacy and as promising tools or targets for new therapeutic treatments. In addition, other noncoding RNAs (long noncoding RNAs and circular RNAs) may play important roles either in CRC-related gene regulation or in acting as miRNA regulators.

In this review, we first briefly analyze the colonic crypt organization and the mechanisms of colorectal carcinogenesis, followed by an initial description of the different types of noncoding RNAs as new molecules involved in the 
regulation of gene expression. We then analyze the main type of regulatory RNAs, miRNAs. First, we introduce their general characteristics and their biogenesis pathway that leads to biologically active miRNA-induced silencing complexes (miRISC). Later on, we present the current knowledge on the involvement of noncoding RNAs in CRC, beginning with the dysregulation of the expression of specific miRNAs, the main signaling pathways involved, and the importance of miRNA polymorphisms in CRC risk. The role of long noncoding RNAs and circular RNAs in CRC is also discussed, with emphasis in the regulation of miRNA function. Finally, we present colorectal cancer treatments and biomarkers, discussing first the classical therapies and potential biomarkers to predict the response to these treatments, followed by an analysis of the use of miRNAs as diagnostic and prognostic markers, their involvement in chemo- and radioresistance, and ending by discussing their potential use as therapeutic targets and tools in CRC.

\section{Colonic Crypt Organization}

The adult colonic epithelium, a single sheet of columnar epithelial cells supported by the lamina propria, has a well-defined architecture organized into crypts, which are dynamic structures that are constantly self-renewing (it is replaced every five days) [16]. The homeostatic self-renewal of the intestine depends on a complex network of interplay involving many cellular processes, including proliferation, differentiation, migration, and apoptosis. All these phenomena are finely coordinated by different signaling pathways (Wnt, Notch, Ephrin, or antagonists of BMP), playing a critical role in the Wnt signaling cascade. Wnt signaling ligands are thought to be produced by mesenchymal cells of the myofibroblast lineage closely attached to the basal lamina that surrounds the crypt.

Three differentiated epithelial lineages mediate colonic function: the colonocytes, or absorptive enterocytes, the mucus-secreting goblet cells, and the less abundant enteroendocrine cells. Each crypt unit is maintained by multipotent stem cells (SCs), located at the bottom of the crypt. During asymmetric division, SCs undergo self-renewal and generate a population of transit-amplifying cells, or progenitors, that, upon migration upwards the crypt, proliferate and differentiate into one of the epithelial cell types of the intestinal wall. These cells, enterocytes, goblet cells, and enteroendocrine cells, continue migrating upwards along the villus until they reach the villus tip where they undergo apoptosis and are shed into the lumen of the intestine [17].

Transit-amplifying cells and stem cells occupy the lower two-thirds of colonic crypts [16]. The terminally differentiated cells, which are found in the top third of the crypt, are continually extruded into the lumen. Adult SCs are defined by self-renewal, potential for multilineage differentiation, and tissue regeneration [18]. The "stem cell zone" model states that small undifferentiated cycling cells (the crypt base columnar cells) are the true intestinal SCs [17].

\section{Colorectal Carcinogenesis}

Colorectal cancer, attending to incidence and mortality statistics, is the third most common form of cancer in men and the second among women. It is one of the main causes of cancer-related death in the more developed countries, leading to an incidence of 1.4 million cases and 693,900 CRC-related deaths occurred in 2012 [19] (http://globocan.iarc.fr). The incidence and mortality from CRC are markedly lower in the less developed countries. Epidemiological studies have shown incidence variations between areas that may be partially explained by varied cultures and lifestyles in different countries and regions. Factors as the lack of dietary fibers, smoking, overweight, obesity, physical inactivity, red and processed meat consumption, and excessive alcohol intake are potential risks for CRC. Around a quarter of CRC patients are incurable at diagnosis and half of those who undergo potentially curative surgery will ultimately develop metastatic disease. Despite the emergence of new targeted agents, early CRC screening, and the use of improved treatments and various therapeutic combinations, none of the available treatments is curative in patients with advanced cancer. Thus, although the application of some medical procedures, such as the use of colonoscopies or the fecal occult blood test, contribute greatly to the early diagnosis and CRC treatment, the deaths from CRC have decreased in several western countries, but its mortality increased in others, such as some East Asian or Latin American countries.

CRC is a multifactorial disease, a complex multistep process involving genetic background, numerous genetic alterations, and environmental influences [5]. Tumors are formed by a heterogenic pool of cells with distinct differentiation patterns. Several studies have been directed towards the identification of markers associated with the initiation and progression of this type of tumors, which normally involve multisequential steps along the adenoma-to-carcinoma transition. The accumulation of mutations in key genes, involving the inactivation of tumor suppressor genes and oncogene activation, follows the progression of the disease. Histopathological and molecular analyses are conducted by Fearon and Vogelstein to formulate, in the 1990s, the "adenomacarcinoma model" or sequence of tumor progression [20]. Thus, the neoplastic process, initiated by APC (Adenomatous Polyposis Coli) or CTNNB1 ( $\beta$-catenin) mutations, results from the sequential mutation of other genes, such as KRAS and TP53, in the context of a growing genomic instability.

The mechanisms of colorectal carcinogenesis have been extensively described $[1,3-5,21]$. The two major types of genomic instability found in colorectal cancers are chromosomal instability (CIN) and microsatellite instability (MSI). CIN is often associated with mutational inactivation of $A P C$ gene. The inactivation of $A P C$ is found in about $85 \%$ of sporadic CRC and is mutated in the germline of patients with FAP (Familial Adenomatous Polyposis). This pathway often includes activation of oncogenes such as COX2 and KRAS and inactivation of additional tumor suppressor genes such as DCC/DPC4 (Deleted in Colon Cancer/Deleted in Pancreatic Cancer, locus 4) and TP53. 
MSI is due to defective/inactivation of DNA mismatch repair $(M M R)$ genes. Mutations in these genes cause genetic defects in other genes that are involved in growth control and behave like tumor suppressor genes. Lynch syndrome and hereditary nonpolyposis colorectal cancer (HNPCC) syndrome are due to inherited mutations in one of the mismatch repair genes (including $M L H 1, M S H 2$, and PMS2). $M M R$ mutations are found in approximately the remaining $15 \%$ of sporadic CRC $[1,3]$. In the $\mathrm{CpG}$ island methylator phenotype, a number of genes become transcriptionally silenced because of hypermethylation of their promoters, and this represents a key epigenetic mechanism of inactivation of tumor suppressor genes, including certain DNA repair genes [1].

The progression of carcinomas to invasive and metastatic disease may involve localized occurrences of a process known as the epithelial-mesenchymal transition (EMT). EMT results in epithelial cells becoming spindle shaped, with loss of cellular polarity similar to mesenchymal cells. These phenotypic changes correlate with increased cellular motility and invasion ability, more characteristic of mesenchymal cells. EMT in metastatic colorectal carcinoma has been studied to identify molecular events that contribute to disease progression [22]. Although loss of E-cadherin function is an initial event in EMT, the expression of specific integrins such as $\alpha 5 \beta 6$ as a consequence of EMT enables invasive cells to interact with interstitial matrices and to sustain activation of TGF- $\beta$ (Transforming Growth Factor- $\beta$ ). EMT also induces autocrine signaling involving VEGF and Flt-1 (its receptor) enabling invasive cells to become self-sufficient for survival. Recent research has demonstrated a convincing link between EMT and cancer stem cells (CSC) as well as their association with CRC progression and therapeutic resistance [23].

It is well known that tumors are composed by a heterogeneous population of cells differing in morphology, marker expression, proliferation ability, and tumorigenic potential. But, what is the origin of the tumor cells? Traditional models of carcinogenesis assumed that this heterogeneity could be explained by stochastic genetic events and microenvironmental influence leading to clonal selection. However, the stochastic theory for the cellular origin of cancer, based upon the assumption that all cancer cells are equally malignant and able to give rise to tumors, has been changed in favor of the hierarchical theory. The latter assumes that tumor cells are hierarchically organized and only a rare subpopulation of undifferentiated cells at the apex of this hierarchy have the unique biological properties necessary for tumor initiation, maintenance, and spreading [24]. Evidence is increasingly supporting the idea that human cancers can be considered as a stem cell disease. According to the CSC model, malignancies originate from a small fraction of cancer cells that show self-renewal and pluripotency and are capable of initiating and sustaining tumor growth [25]. In this model, the stem cell-like cells (CSC or tumor-initiating cells) are capable of propagating a tumor in the same way as normal stem cells control proliferation and differentiation in normal tissue [18]. In fact, it is now accepted that CSC can originate from mutations in normal somatic stem cells that deregulate their physiological programs. Alternatively, mutations may target more committed progenitor cells or even mature cells, which become reprogrammed to acquire stem-like functions. In any case, mutated genes should promote expansion of stem/progenitor cells, thus increasing their predisposition to cancer development by expanding self-renewal and pluripotency over their normal tendency towards relative quiescence and proper differentiation [26].

In summary, recent evidence points out that cancer can be considered a disease in which mutations either convert normal stem cells into aberrant counterparts or induce more differentiated cells to revert towards a stem cell-like behavior. This has major implications for the development of new targeted therapeutic strategies aimed at eradicating the tumor stem cell population. In fact, CSCs exhibit higher resistance to cytotoxic drugs and radiation as compared to bulk tumor cells. Classical therapeutic approaches may preferentially kill more differentiated cells while sparing CSCs. Survival of even few CSCs would later result in subsequent tumor regrowth and disease relapse [27].

The identification CSC populations in human colon tumors was first published in 2007 by two independent groups $[28,29]$. This subset of cells, identified as $\mathrm{CD}_{133^{+}}$, was able to initiate tumor growth in murine xenograft models $\left(\sim 1\right.$ in $262 \mathrm{CD} 133^{+}$colon cancer cells represented a CSC) [28]. The expression of CD133 has also been demonstrated in some tumor cell lines, including Caco-2 cells from human colon adenocarcinoma [30], and in colorectal tumors and cells derived from them $[28,29]$. Moreover, CD44 and CD166 expression can also be used to enrich for a CSC subset in colon cancers [31]. The use of several cell-surface markers, such as CD44, CD24, CD133, CD166, EpCAM (epithelial cell adhesion molecule), and ABCB5 (ATP-binding cassette subfamily B member 5), has successfully allowed the isolation of CSCs from solid tumors, including human colon, breast, brain, pancreatic, liver, ovarian, and melanoma. A number of markers used in cell sorting are emerging as being predictive of disease progression, indicating that they identify clinically important cell populations.

Other aspect that was considered in CRC is gene hypomethylation, which was first described in 1983 by Feinberg and Vogelstein [32]. Since then, several epigenetic abnormalities have been reported, mainly in the first stages of CRC development, while only few have been found to happen at more advanced stages (adenocarcinoma and metastasis) [33, 34]. Hypermethylation at gene promoter or transcription start region has been described to be cause of gene transcription silencing during CRC development, and many of the affected genes normally exhibited tumor suppressor roles. In contrast, hypomethylation activates genes that are normally silenced and results in global chromosomal instability [35]. Altered acetylation or methylation of histones has also been reported in CRC affecting the expression of tumor-associated genes and cell cycle progression. However, this epigenetic modification is gene-specific and a general pattern has not been found. HDAC inhibitors as butyrate, TSA (Trichostatin A), or SAHA (suberanilohydroxamic acid, also known as Vorinostat) induce not only hyperacetylation but also hypermethylation of histones altering the expression of genes; among them, several ones are involved in tumor 
development and cell cycle and arresting cells in $G_{1}$ phase [36-39]. Some cells may even become resistant to HDAC inhibitors and develop an overall resistance to different types of stress enhancing their malignant potential, as has been described for colon carcinoma cells [40-44]. Additionally, SC may become CSC under a series of epigenetic and genetic alterations. Both hypermethylation and hypomethylation of key genes are involved in epigenetic changes during SC malignant transformation [35]. Moreover, the fully differentiated somatic cells can also acquire CSC-characteristics through massive genetic and epigenetic reprogramming. The CSCs then give rise to and maintain the heterogeneous tumor mass [45].

\section{New Players in Gene Expression Regulation: Noncoding RNAs}

4.1. The Discovery of the Noncoding RNAs. In humans, about $2-3 \%$ of transcripts have the capacity to encode protein and approximately 20,000-25,0000 genes encode proteins, whereas many noncoding elements are transcribed into noncoding RNA (ncRNA). However, functional ncRNAs may arise from only a small fraction of the total genome. These ncRNAs are a class of RNA molecules that are involved in the regulation of biological processes, including gene expression, epigenetic processes, cell differentiation, proliferation, migration and apoptosis, and transcriptional and posttranscriptional regulation, and are implicated in different human diseases. Due to the role of ncRNAs in such key processes, they are a focus of research interest and several excellent reviews on this topic have been published [9, 46-49]. However, further research efforts are necessary to explore the regulatory functions of this novel class of RNA molecules.

In the 1970s, researchers began to realize that the genome was transcribed into nonprotein-coding RNAs, as the known housekeeping ribosomal and transfer RNAs (rRNAs and tRNAs), and the role of other ncRNAs in gene expression regulation was postulated. In the 1980s, small nucleolar RNAs (snoRNAs; intermediate-sized ncRNAs of 60-300 nt) and small nuclear RNAs (snRNAs; average length approximately $150 \mathrm{nt}$ ) became recognized as major players in posttranscriptional RNA processing. When were ncRNAs first described? Introns, that were discovered in 1977 [50], accounted for a part of the noncoding sequences. However, for more than a decade, researchers paid very little attention to what happened with the intronic RNA fragments after their removal from the pre-mRNA. It was not until 1990 that Liu and Maxwell described that the intronic sequences of the mouse hsc70 heat shock gene (HSPA8) were the source for U14 snoRNA [51]. These molecules are primarily involved in chemical modifications of other RNAs, mainly rRNAs, tRNAs, and snRNAs. In this process, the complementary pairing of their guide sequences with that of the RNA target is essential. There are two main classes of snoRNA, the C/D box snoRNAs, which are associated with methylation, and the H/ACA box snoRNAs, which are associated with pseudouridylation. snoRNAs are components of the small nucleolar ribonucleoproteins (snoRNPs), which are complexes responsible for the two aforementioned sequencespecific modifications: $2^{\prime}$-O-methylation and pseudouridylation. Many of the newly discovered snoRNAs are synthesized by an intron-processing pathway, which provides a potential mechanism for coordinating nuclear RNA synthesis. For example, posttranscriptional modifications of rRNAs take place in the nucleolus (the nuclear compartment within which ribosomes are formed) and facilitate rRNA folding and stability [52].

The functions of ncRNAs are quite variable and specific. For example, studies on snRNAs have shown that their primary function is the processing of pre-mRNAs (heterogeneous nuclear RNA or hnRNA) in the nucleus. The 7SKRNA plays a role in transcription regulation by controlling the positive transcription elongation factor P-TEFb. It is found in a small nuclear ribonucleoprotein complex (snRNP) with a number of other proteins that regulate the stability and function of the complex. On the other hand, the 7SLRNA is a component of the eukaryotic signal recognition particle together with six distinct polypeptides. The ribozyme ribonuclease $\mathrm{P}$, that cuts the leader $5^{\prime}$ sequences of all tRNAs, is an example of catalytic RNA. The impact of all of these topics in which ncRNAs are involved has been reflected in three Nobel Prizes. In 1989, the Nobel Prize in Chemistry was awarded jointly to Sidney Altman and Thomas R. Cech "for their discovery of catalytic properties of RNA." In 2006, the Nobel Prize in Physiology or Medicine was awarded jointly to Andrew Z. Fire and Craig C. Mello "for their discovery of RNA interference - gene silencing by double-stranded RNA." In 2009, the Nobel Prize in Chemistry was awarded jointly to Venkatraman Ramakrishnan, Thomas A. Steitz, and Ada E. Yonath "for studies of the structure and function of the ribosome."

According to size, the ncRNAs have been systematically classified into two groups, small ncRNAs (sncRNAs) and long ncRNAs (lncRNAs), which are shorter than 200 or longer than $200 \mathrm{nt}$, respectively. The sncRNAs can be divided into microRNAs (miRNAs), small interfering RNAs (siRNAs), snoRNAs, snRNAs, PIWI-interacting RNAs (piRNAs), ribozymes, and telomere-associated RNAs (TERC), circular RNAs (circRNAs), and other sncRNAs. The classification of lncRNAs is based on different parameters, as genomic location, effect exerted on DNA sequences, mechanism of action, and their targeting mechanism [47]. In addition, they can be classified according to their position in the genome into intergenic (lincRNA), intronic, bidirectional, sense, and antisense lncRNAs. Several studies have revealed that both small and long ncRNAs can regulate gene expression at the transcriptional, posttranscriptional, and epigenetic levels. Among the former, miRNAs, endogenous siRNAs, and piRNAs have been described to exert a gene regulation role. Although only a few lncRNAs have been characterized to date, recent work has revealed the regulatory role of lncRNAs. In addition to their length, known lncRNAs have key differences with small ncRNAs and some intriguing similarities to mRNAs. As we describe later on, small ncRNAs regulate gene expression by sequence-specific binding, but lncRNAs do it through diverse mechanisms that remain unclear $[9,53]$. 
In 1998, the Nobel Laureates Andrew Fire and Craig Mello described a key mechanism for controlling the flow of genetic information. They demonstrated the potent and specific genetic interference by exogenous double-stranded RNA in Caenorhabditis elegans, which was more effective in producing interference than either strand individually [54]. Such effects have been proposed to result from a simple antisense mechanism that depends on hybridization between the injected RNA and endogenous messenger RNA transcripts. In this process, gene expression is inhibited by degradation of specific mRNA molecules. The mechanism underlying RNA interference and components of the RNA silencing machinery were identified during the following years. Thus, two types of sncRNAs, siRNA and miRNA, have attracted considerable attention because they play an important role in gene regulation and have a therapeutic potential in the treatment of many different diseases including cancer [55].

In the last years, growing experimental evidences suggest that alterations in gene-regulator ncRNAs are relevant in tumorigenesis and that most human tumors are characterized by dysregulation of miRNAs. In this review, we focus on miRNAs, which are considered the main regulatory molecules among ncRNAs and have been shown to play an important role in CRC and also in the participation of lncRNAs as well as circRNAs, both regulating miRNA activity.

4.2. MicroRNAs: The Main Noncoding Regulatory RNAs. miRNAs are naturally occurring small noncoding, singlestranded RNA molecules of approximately 22 nucleotides in length that are derived from hairpin precursors. MiRNA are well characterized as a large class of gene regulators and are estimated to regulate the translation of more than $60 \%$ of protein-coding genes [9]. They negatively regulate gene expression by binding to complementary sequences within target mRNAs for posttranscriptional gene silencing. This binding occurs mainly within the $3^{\prime}$ - untranslated region ( $3^{\prime}$ UTR) of target mRNA based on sequence complementarity and results in target mRNA translational repression or mRNA degradation [56,57]. In addition, some miRNAs have been shown to bind to the open reading frame or to the $5^{\prime}$ UTR of the target mRNAs. miRNAs are a class of highly abundant noncoding RNA molecules that are involved in several biological processes, as cell proliferation and differentiation, and in many diseases including cancer.

Since the initial discovery of lin- 4 and let-7 miRNAs, which are components of the gene regulatory network that controls the timing of larval development in C. elegans, a plethora of miRNAs has been reported. The association of miRNAs with cancer development was described for the first time after the detection of frequent deletions of miR-15 and miR-16 loci in B-cell chronic lymphocytic leukemia [7, 58]. The hallmarks in miRNA discovery and its relationship with cancer are shown in Table 1. During the last twelve years, several studies describing the correlations between miRNA and cancer and metastasis, their role as molecular biomarkers, and their involvement in the oncogene and tumor suppressor networks have been published [59].

Sequences and annotations of published miRNAs are archived in miRBase database. The first release of miRBase in
2002 contained 218 miRNA loci from five species (Table 1). Since then, the miRNA discovery field has exploded with hundreds of miRNAs found to be present in each studied animal and plant genome. They are extended to the majority of vertebrates and are conserved throughout evolution, from worms to plants and humans. The number of published miRNA sequences in miRBase continues to increase rapidly, mainly driven by small RNA deep sequencing experiments. Thousands of miRNAs across different species have been identified; the exponential evolution of the number of publications along the years is shown in Figure 1(a) as well as the increase in miRNA entries in the miRBase database and number of species where they have been found (Figure 1(b)). The latest version of the miRNA database (release 21; June 2014) contains 28,645 miRNA loci from 223 species and 16 viruses, corresponding at about 35,828 mature miRNAs. This number is even expanding and mirBase 21 contains at the moment 1,881 precursors and 2,588 human mature miRNAs representing $1-3 \%$ of all human genes [60].

The miRBase also provides rules for the standard nomenclature system of the miRNAs and the criteria for identification and naming miRNAs (http://www.mirbase.org/help/ nomenclature.shtml) [61, 62]. Thus, the numbering of newly identified miRNA genes is sequential and each name is preceded by abbreviated three-letter prefixes to designate the species; for humans, those letters are "hsa" (Homo sapiens). The mature sequences are designated as "miR," whereas both the gene locus and precursor hairpins (premiRNA) of a miRNA are identified as "mir." However, names referring to genomic loci should be written in italics for easier differentiation from mature sequences. miRNAs with nearly identical sequences except for one or two nucleotides are annotated with an additional lowercase letters. For example, hsa-miR-200b is closely related to hsa-miR-200c (Figures 2(a) and 2(b)). Additionally, miRNAs from genes located in different genome regions that lead to an identical mature miRNA are indicated with an additional number (e.g., miR135a-1 and miR-135a-2). When both strands of the hairpin structure of a pre-miRNA are processed as mature miRNA, an indication must be given to specify which arm generates one or the other of the two miRNAs. Such mature sequences are named followed by $-5 p$ and $-3 p$ (e.g., miR-141-5p and miR-141$3 p$; Figure 2(c)). Finally, when the relative abundancy clearly indicates which is the predominantly expressed miRNA, the mature sequences are assigned names of the form miR-56 (the predominant product) and miR-56* (from the opposite arm of the precursor and nonfunctional). However, when the data are not sufficient to determine which sequence is the predominant one, or the functionality of a miRNA is described, names like miR-141-5p (from the $5^{\prime}$ arm) and miR$141-3 p$ (from the $3^{\prime}$ arm) are recommended. Finally, attending to the function of miRNAs, some researchers refer to specific miRNAs as ts-miRNAs (tumor suppressor), oncomiRs (oncogenic), and epi-miRNAs (subgroup that modulate the epigenetic machinery [9]).

Several studies have indicated that miRNAs could be grouped in "seed families" based on sequence homology at the $5^{\prime}$ end of the mature miRNA. This $5^{\prime}$ end is crucial for the stability and proper loading of the miRNA into 
TABLE 1: Hallmarks in miRNA discovery and its relationship to cancer.

\begin{tabular}{ll}
\hline Year & Hallmark \\
\hline & The first regulatory noncoding RNA was identified: lin-4 \\
Two discoveries identified a novel mechanism of posttranscriptional gene regulation. The first miRNA lin-4 was described in \\
1993 as asmall temporal RNA (stRNA) in the laboratory of Ambros working with the nematode Caenorhabditis elegans. In this \\
system, the transition from the first to the second larval stage fates requires the 22-nucleotide lin-4 RNA. The gene lin-4 \\
encodes a small RNA, which is a non-protein-coding regulatory RNA molecule. In the same issue of cell, the group of Ruvkun \\
reported the first miRNA target gene, the heterochronic gene lin-14, that is regulated by lin-4 mediating the temporal pattern \\
formation in C. elegans. The sequence of lin-4 has antisense complementarity to lin-14 mRNA that encodes lin-14 protein \\
{$[80,81]$.}
\end{tabular}

The second small temporal RNA and the first in humans was identified: let-7

Seven years later the second regulatory stRNA, let-7, was discovered. The transition from late larval to adult cell fates in $C$. elegans requires the 21-nucleotide let-7 RNA. This stRNA negatively regulates, among others, lin-14 and lin-28 through

2000 RNA-RNA interactions with their $3^{\prime}$ untranslated regions. The sequential stage-specific expression of the lin-4 and let-7 RNAs regulates the timing of $C$. elegans development. The let-7 RNA showed its conservation across species, including $H$. sapiens. In humans, let-7 was detected at different expression levels in several tissues, including brain, heart, kidney, liver, lung, trachea, colon, small intestine, spleen, stomach, and thymus [82, 83].

The term miRNA is introduced

In addition to lin-4 and let-7, several stRNAs with regulatory functions were discovered using bioinformatics and cDNA

2001 cloning. Three papers published in the same issue of Science showed the existence of small RNAs involved in posttranscriptional regulation of target mRNA in vertebrates and invertebrates. These RNAs were named microRNAs (miRNA) to refer to this class of small regulatory RNAs [84-86].

miRNA is associated with cancer

The relevance of miRNAs to cancer was suggested by changes in their expression patterns and recurrent amplification and deletion of miRNA genes in tumors. The first report suggesting a role of miRNAs in cancer described a frequent 13q14 deletion 2002 that encoded the miRNA-15a/16-1 cluster reducing its expression in chronic lymphocytic leukemia. Both genes were deleted or downregulated in $68 \%$ of analyzed cases. Two years later, the same group found that a significant percentage of miRNAs is located at fragile sites and in genomic regions altered in cancers, including regions of amplification or loss of heterozygosity or breakpoints. They suggested that miRNAs were a new class of genes with a relevant role in human cancer pathogenesis [7,58].

miRNAs in colorectal cancer

A total of 28 different miRNA sequences were identified in a colonic adenocarcinoma and normal mucosa. Among them, miR-21, miR-143, miR-145, and miR-200c were expressed. In colorectal cancer, two different miRNAs, miR-143 and miR-145

2003 exhibited significantly reduced levels of the mature miRNAs compared to normal mucosa specimens. The maintenance of constant levels of unprocessed hairpin precursors in both normal and tumor tissues suggested that altered transcription is not responsible for reduced miRNA levels. Authors proposed that the reduction is due to posttranscriptional processes such as a reduced Dicer-processing activity in the neoplastic cells or reduced stability of these specific miRNAs [87].

miRBase: the miRNA sequence database

miRBase was established in 2002 as a miRNA registry. The criteria for the identification of miRNAs was published in 2003.

2002-2003 The miRBase grew from the miRNA registry resource set up by Griffiths-Jones in 2003 and is the public repository for all published miRNA sequences and annotation data. Its aim is assigning stable and consistent names to newly discovered miRNAs. The first release of miRBase in 2002 contained 218 miRNA loci from five species. Since then, the number of miRNAs discovered has increased exponentially. The miRBase is freely available at http://www.mirbase.org/ [60-62].

miRNAs as molecular biomarkers

let-7 expression is associated with survival of lung cancer patients. This was the first time that miRNAs are suggested as prognostic markers. The article described that let-7 expression was reduced in lung cancers and that lung cancer patients with low let-7 expression levels had a significantly shorter survival after potentially curative resection. Currently, the clinical utility of miRNAs as diagnostic/prognostic biomarkers has been demonstrated in several types of cancer by numerous studies using tumor samples [88].

Function of miRNAs in cancer

The first reports addressing the biological function of miRNAs in cancer were published. These articles described that miR-15 and miR-16, the first two miRNAs associated with cancer, play a role in apoptosis regulation by targeting the antiapoptotic

2005 bcl-2 mRNA. They also reported the first miRNA-target interaction with relevance to cancer: human Ras expression is regulated by let-7 in cell culture. In fact, let-7 expression is decreased in lung cancer compared with normal tissue, and it correlates with the increased Ras protein levels detected in lung tumor samples. Since then, hundreds of publications have reported on the role of miRNAs in tumors [89-91].

The expression of miRNAs is regulated by transcription factors

2005 It is described that c-Myc activates the expression of a cluster of six miRNAs on human chromosome 13. In turn, the expression of a target of $\mathrm{c}-\mathrm{Myc}$, the transcription factor E2F1, is negatively regulated by two oncogenic miRNAs in this cluster, miR-17-5p and miR-20a [92]. 
TABLE 1: Continued.

\begin{tabular}{|c|c|}
\hline Year & Hallmark \\
\hline 2005-2007 & $\begin{array}{l}\text { Role of miRNAs as candidate components of oncogene and tumor-suppressor networks } \\
\text { The role of miRNAs as oncogenes (oncomiRs) or tumor suppressors involved in a variety of pathways deregulated in cancer } \\
\text { was reported. The polycistronic miRNA cluster miR-17-92, located in a region of DNA that is amplified in human B-cell } \\
\text { lymphomas, is reported as a potential human oncogene. Other studies, using different types of tumors, also described the role } \\
\text { of miR-143, miR-145, miR-372, miR-373, and miR-155/BIC as oncogenic miRNAs. Conversely, five independent reports } \\
\text { describe that the miR-34 family of evolutionarily conserved miRNAs are directly induced by p53 in response to DNA damage } \\
\text { and oncogenic stress. miR-34a was identified as a miRNA component of the p53 network, revealing an interplay between } \\
\text { proteins and noncoding RNAs in a tumor-suppressor pathway [93-102]. }\end{array}$ \\
\hline 2007 & $\begin{array}{l}\text { miRNAs "sponges" } \\
\text { The initial term "target mimicry" was coined in plants to define the mechanism of inhibition of miRNA activity discovered } \\
\text { studying the phosphate homeostasis in Arabidopsis thaliana. In the same year, there is a report in humans on specific } \\
\text { competitive inhibitors from transcripts expressed from strong promoters that contain multiple tandem binding sites to several } \\
\text { miRNA seed families; they were named "miRNA sponges" }[103,104] \text {. }\end{array}$ \\
\hline 2007-2009 & $\begin{array}{l}\text { miRNAs and metastasis } \\
\text { miRNAs are also involved in metastasis; they can promote or inhibit metastasis. The first miRNA described as a metastasis } \\
\text { activator was miR-10b, that positively regulates cell migration and invasion in vitro and is capable of initiating tumor invasion } \\
\text { and metastasis in vivo. Expression levels of miR-10b in primary breast carcinomas correlate with clinical progression. Its } \\
\text { expression is elevated in about } 50 \% \text { of metastatic breast tumors compared with metastasis-free tumors or normal breast } \\
\text { tissues. Human miR-373 and miR-520c also stimulate cancer cell migration and invasion in vitro and in vivo. On the contrary, } \\
\text { other miRNAs can prevent tumor metastasis. Breast cancer patients with low expression levels of miR-335, miR-126, and } \\
\text { miR-206 had a shorter median time to metastatic relapse. Restoration of their expression in breast cancer cell lines decreased } \\
\text { the number of metastases in inoculated mice [105-108]. }\end{array}$ \\
\hline 2008 & $\begin{array}{l}\text { Circulating miRNA biomarkers } \\
\text { miRNAs are detected in blood samples (plasma, platelets, erythrocytes, and nucleated blood cells). This pointed out that } \\
\text { endogenous plasma miRNAs are protected in some manner to prevent their degradation. Due to their stability in the } \\
\text { circulation, miRNAs began to be considered for their potential use as biomarkers for different pathologies [109-112]. }\end{array}$ \\
\hline 2013 & $\begin{array}{l}\text { miRNA therapeutics } \\
\text { miR-34a mimic (MRX34) enters Phase } 1 \text { clinical study in liver cancer and other solid tumors with liver involvement, as well as } \\
\text { hematological malignancies. Regretfully, this study was halted in } 2016 \text { due to immune-related serious adverse events [113]. }\end{array}$ \\
\hline
\end{tabular}

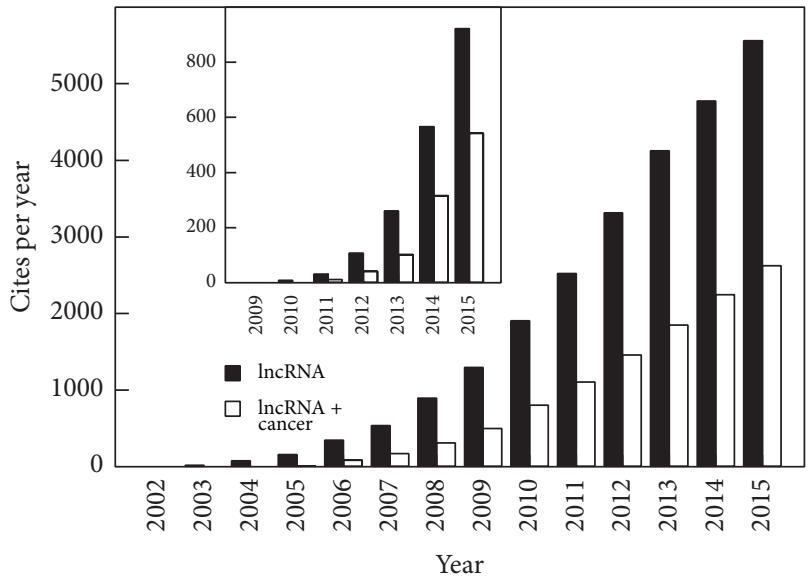

miRNA

miRNA + cancer

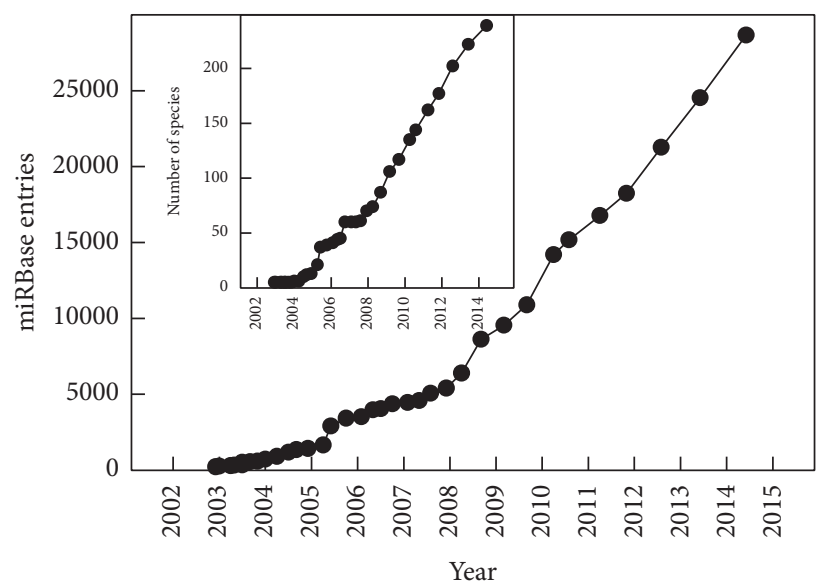

(b)

FIGURE 1: Evolution of the number of publications on miRNAs and lncRNAs. (a) The number of publications per year (searched in PubMed) that contain the terms "microRNA" (black bars) and "microRNA + cancer" (empty bars) in their title, abstract, or keywords, is shown. The inset is equivalent with the terms "IncRNA" and "lncRNA + cancer". (b) Evolution of the number of entries in miRBase from December 2002 until the last release (release 21) I June 2014. The inset shows the increase in the number of species where miRNAs have been found. 


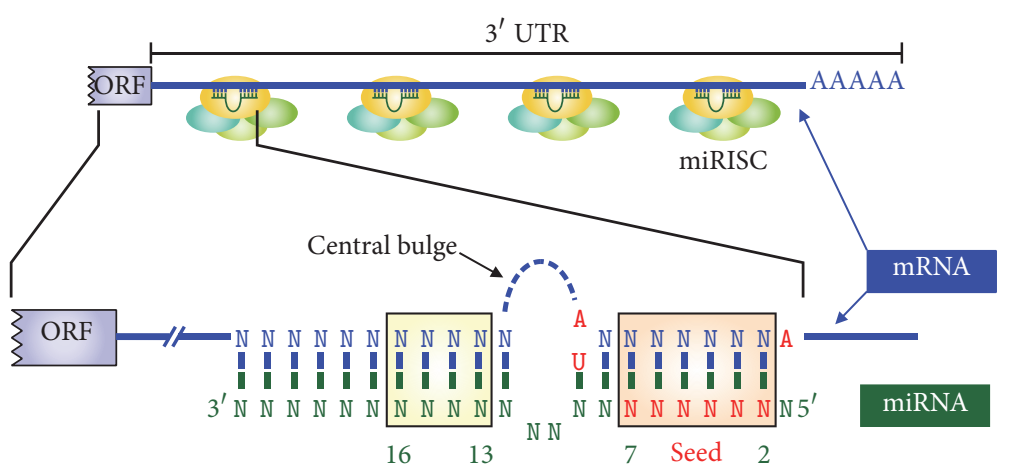

(a)
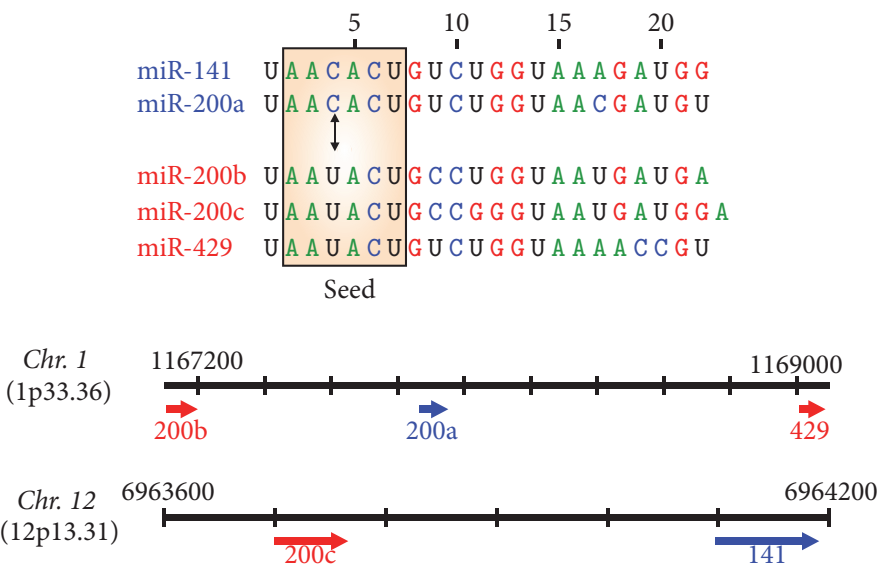

(b)

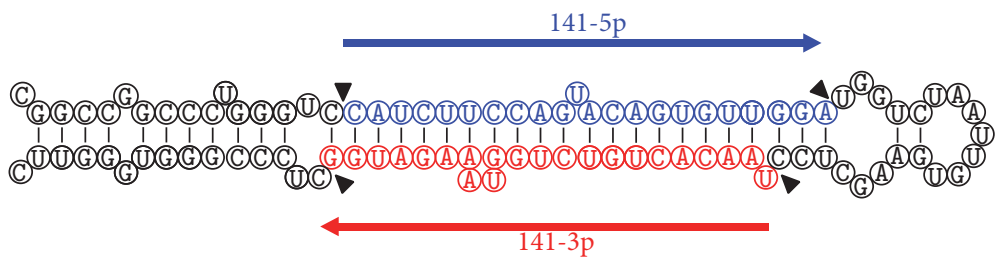

(c)

FIGURE 2: Interaction between miRNAs and target mRNAs in animals and miRNA-200 family. (a) miRNAs (green) target mRNAs (blue) in the $3^{\prime}$ UTR. This mRNA region can bind one or various miRISC complexes arising from the same or different miRNAs. A perfect complementarity is found in the seed region between nucleotides 2 and 7 from the $5^{\prime}$ end of the miRNA (orange box). A central bulge prevents endonucleolytic cleavage mediated by Ago2 (a major difference with the miRNA-target mRNA interaction in plants). A few nucleotide matches in the miRNA $3^{\prime}$ end (especially between nucleotides 13 and 16; yellow box) are necessary for the best stabilization of the miRNA/mRNA duplex. The presence in the mRNA sequence of an A residue in position 1 and/or an A or U residue in position 9 can increase miRNA efficiency [6]. (b) The miR-200 family of miRNAs consists of two closely related subfamilies that differ in one nucleotide within the seed sequence (boxed). The five miR-200 family members are located on two different genetic loci in chromosomes 1 and 12. (c) Schematic representation of the secondary structure of the pre-miR-141 hairpin. The sequence of the mature miR-141-3p is indicated in red and the miR-141-5p (previously called miR-141*) is in blue. Black triangles show Dicer cleavage sites due to its RNase III activity.

the miRNA-associated multiprotein RNA-induced silencing complex (miRISC). A schematic model of one of the canonical interactions between miRNAs and $3^{\prime}$ UTR target mRNAs is shown in Figure 2(a). A perfect complementarity is found primarily in the seed region between nucleotides 2 and 7 from the $5^{\prime}$ end of the miRNA. A few nucleotide matches in the miRNA $3^{\prime}$ end (nucleotides 13 to 16) are necessary for the best stabilization of the miRNA/mRNA target duplex [6]. Other categories of target sites, as marginal or atypical sites, have also been reported according to their structural features.
Therefore, the targets of a miRNA family are likely to overlap among members. In addition, a single mRNA can be targeted by multiple miRNAs, and an individual miRNA can have more than one mRNA target. Those mRNAs that share the same miRNA response elements, or bind members from the same miRNA family, are reported to influence the expression of each other by competing for miRNA binding. Interestingly, the mRNA-miRNA interaction network is complex; single miRNA might regulate and bind to as many as two hundred mRNA targets. The proteins corresponding to these targets 
can be diverse in their function; they include transcription factors, secreted factors, receptors, and transporters [63]. The chromosomal localization and seed sequences corresponding to the miR-200 family members, which comprise five miRNAs, miR-200a, -200b, -200c, miR-141, and miR-429, are shown in Figure 2(b). The secondary structure of the premiR-141 hairpin and the origin and sequences of miR-141-5p and miR-141-3p are presented in Figure 2(c).

4.3. MicroRNA Biogenesis. Eukaryotic nuclear DNA encodes miRNAs and their genomic organization is diverse. They can be located within exons or introns, in intergenic sequences or produced from lariat introns $[64,65]$. Exonic miRNAs are located within exons and are independently transcribed from their own promoters into precursor pri-miRNAs. When some miRNA genes overlap protein-coding genes in the antisense direction, they also have their own promoters. In mammalian cells, more than $50 \%$ of miRNAs are found within an intron of a protein-coding gene. They may be under the control of their own independent promoter or may be cotranscribed with the host gene and processed into pre-miRNAs subsequent to intron splicing. In addition, some miRNA genes are intergenic and their transcription is driven by their own upstream promoter and may be either RNA polymerase II-dependent or III-dependent. Moreover, mirtrons are small lariat intronderived precursor miRNAs excised by the splicing machinery. Following splicing, mirtrons undergo debranching by a lariat-debranching enzyme and then fold into hairpin structures resembling precursor miRNAs. Mirtrons are transported to the cytoplasm by exportin-5/Ran-GTP following the typical miRNA-processing pathway presented below. Additionally, an individual pri-miRNA can either produce a single miRNA or contain clusters of two or more miRNAs that are processed from a long primary transcriptional unit. In this way, the largest gene cluster of human miRNAs is located in chromosome 19q13.41. This miRNA cluster (C19MC) encodesmore than 59 mature microRNAs and is exclusively expressed in the placenta and in undifferentiated cells [66].

Biogenesis of miRNAs takes place through a multistep process that involves the activity of RNase III enzymes Drosha and Dicer and ultimately results in the production of mature miRNAs of about $22 \mathrm{nt}$. Several review articles summarize the miRNA biogenesis and its regulation $[10-12,65$, 67-69]. The miRNA biogenesis pathway is shown in Figure 3. The process generally starts with the transcription by RNA polymerase II of the miRNA gene yielding in the nucleus primary large miRNA (pri-miRNA) transcripts which are $5^{\prime}$ capped (m7GpppG) and $3^{\prime}$ polyadenylated [poly(A) tail]. These long pri-miRNA transcripts are subjected to processing by the microprocessor double-stranded RNA (dsRNA) RNase III enzyme Drosha and its cofactor the dsRNAbinding protein DGCR8 (DiGeorge syndrome critical region 8). Drosha recognizes the base of the stem-loop hairpin structure and cleaves the $5^{\prime}$ and $3^{\prime}$ arms of the pri-miRNA, whereas DGCR8 directly interacts with and stabilizes the pri-miRNA and determines the precise cleavage site. Following cleavage of the pri-miRNA molecule by the Drosha microprocessor, its size is reduced to a $60-70 \mathrm{nt}$ pre-miRNA precursor product (pre-miRNA) which contains an imperfect stem-loop hairpin flanked by single-stranded RNA (ssRNA). After nuclear processing, the pre-miRNAs are then exported from the nucleus into cytoplasm by exportin-5/Ran-GTP, a nuclear transport receptor complex. Dicer, another RNase III enzyme, and the transactivation-responsive RNA-binding protein (TRBP or TARBP2) process the pre-miRNA in the cytoplasm to generate a transient $\sim 22 \mathrm{nt}$ miRNA:miRNA* duplex with 2 nt overhangs at the $3^{\prime}$ ends. Next, the RISC loading complex is formed after recruiting Argonaute (AGO) protein. One miRNA strand is the antisense or guide/mature strand, while miRNA* is the sense or passenger strand. This duplex is then loaded into the miRISC, which includes an AGO2 protein, GW182/TNRC6 proteins, Gemin 3/4 (Deadbox helicases DDX20 and DDX42), among other potential proteins, and the mature functional guide single-stranded miRNA yielding a functional miRISC. Usually the passenger strand is cleaved. The mature functional miRNA guides the RNA-induced silencing complex (RISC) to miRNA response elements on target mRNA transcripts to posttranscriptionally negatively regulate gene expression via translation inhibition or transcript degradation [57].

An exception to this general pathway is the maturation of mirtrons. Figure 3 shows that mRNA splicing can produce miRNA-containing introns that are processed by a Droshaindependent mechanism. The lariat intron is debranched by a lariat-debranching enzyme instead of Drosha and, after refolding into hairpin structures, is transported to the cytoplasm by exportin-5/Ran-GTP and follows the typical miRNA-processing pathway. Thus, they are cleaved by Dicer and the mature strands are loaded onto miRISC. Figure 3 also shows the incorporation of the siRNAs into the RNA silencing machinery at the level of Dicer. The complex regulation of miRNA gene transcription as well as the multiple regulators (activators and repressors) of the miRNAs processing have been considered in several reviews $[10,12,68]$.

miRNAs regulate gene expression through multiple pathways in a sequence-specific fashion. As we have already described, the degree of complementarity between the $5^{\prime}$ seed region of the miRNA and its $3^{\prime}$ UTR target mRNA determines the process (Figure 2(a)). In plants, the perfect or near-perfect base pairing often leads to the cleavage of target mRNAs and subsequent gene silencing by RNA interference pathway. In eukaryotic cells, also a perfect pairing between a miRNA and its target site induces endonucleolytic cleavage by Argonaute, leading to rapid degradation of the mRNA. However, the binding of miRNAs to mRNA targets with imperfect complementarity block target gene expression at the level of protein translation [12, 15, 65, 68]. In eukaryotic cells, mRNA translation is stimulated by the formation of circular structures where the $5^{\prime}$ and $3^{\prime}$ ends of mRNAs are connected through the interaction between a complex formed by the eukaryotic initiation factor eIF-4E, that binds the $5^{\prime}$ cap, and the cytoplasmic poly(A)-binding protein (PABPC), brought together by eiF-4G. The latter also interacts with eiF-3, which binds the $40 \mathrm{~S}$ ribosomal subunit and promotes its assembly on the mRNA (Figure 4). The partial pairing of the miRNA complex to target $3^{\prime}$ UTR sites can result in deadenylation of the mRNA through recruitment of the 


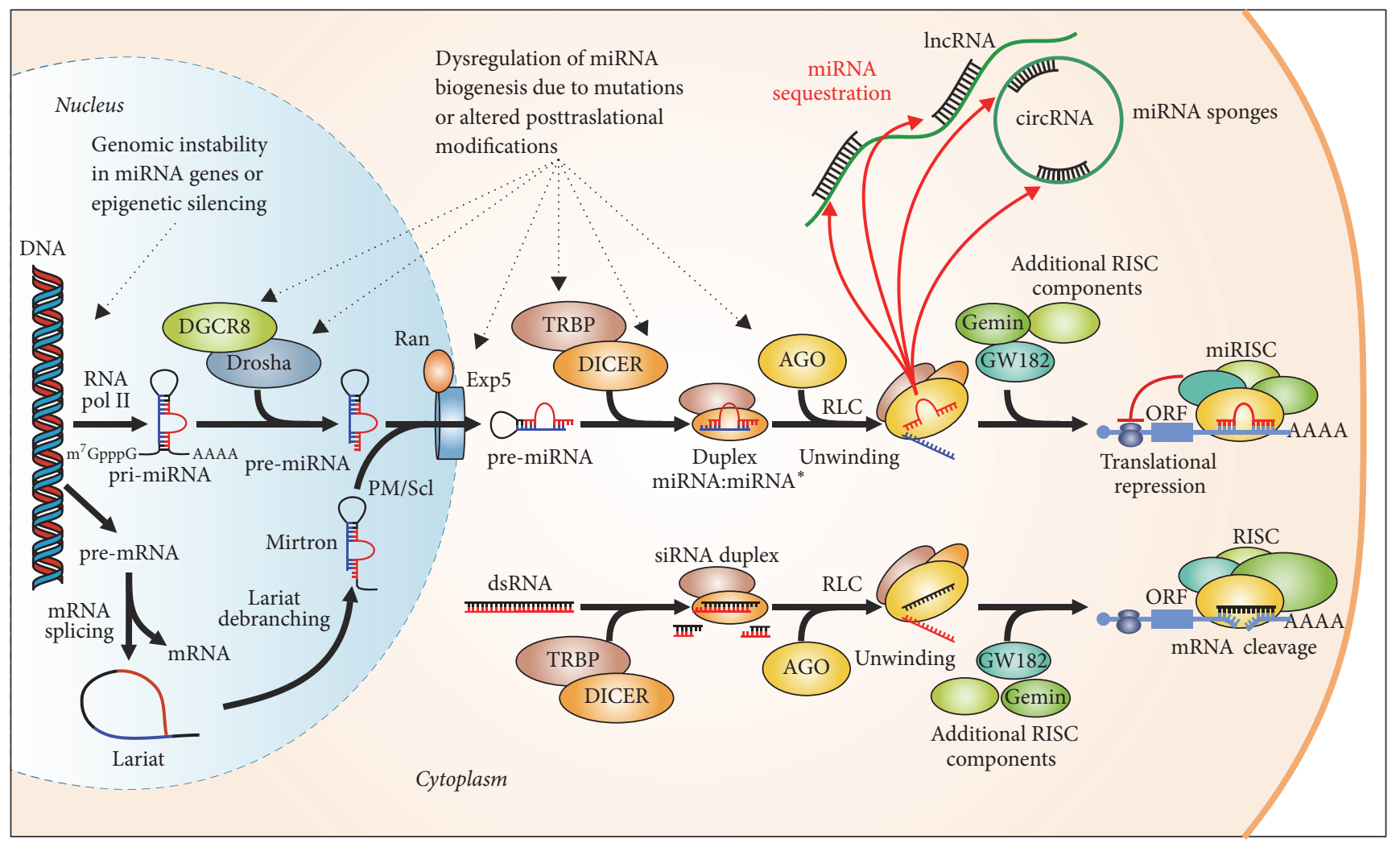

FIGURE 3: Biogenesis of miRNAs and translational repression exerted by miRNAs and siRNAs. The nascent pri-miRNA transcripts are first processed into 70 -nucleotide pre-miRNAs by Drosha/DGR8 complexes inside the nucleus. MiRNAs can also be byproducts of mRNA splicing after lariat debranching and $3^{\prime}$-trimming by the exosome complex PM/Scl (mirtrons). Pre-miRNAs (or mitrons) are transported to the cytoplasm by exportin 5 coupled with Ran-GTP and are processed into miRNA:miRNA* duplexes by Dicer/TRBP. Dicer also processes endogenous or exogenous dsRNA duplexes. Only one strand of the miRNA:miRNA* duplex or the siRNA duplex is preferentially assembled into RISC by the RISC loading complex (RLC). Subsequently, the RISC complex acts on its mRNA target by translational repression or mRNA cleavage, depending, at least in part, on the level of complementarity between the small RNA and its target. Alterations of miRNA function in cancer are multifactorial. They can arise from epigenetic silencing of miRNA genes or may be due to genetic instability as human microRNA genes are frequently located at fragile sites and genomic regions involved in cancers $[7,8]$. Dysregulation of miRNA biogenesis machinery is also frequent in cancer mainly due to mutations in one or several of the proteins involved in processing (Drosha, DGCR8, DICER, TRBP, and Argonaute), or in nuclear export (exportin 5), or by alterations in their posttranslational modifications (PTMs) [7, 9-14]. Although specific miRNAs have been described as acting as oncogenes and tumor suppressors, the miRNA expression profile of human tumors is characterized by a general defect in miRNA production that results in global miRNA downregulation. In addition, miRNA sequestration by the so-called miRNA sponges (i.e., circRNAs and lncRNAs) can also contribute to dysregulation of miRNA function. ORF, open reading frame.

CCR4-NOT or PAN2/3 complexes by the miRISC-associated GW182 proteins. Loss of the poly(A) tail causes dissociation of PABPC and leads to mRNA degradation. Moreover, the miRISC can also induce translational repression by blocking initiation via recruitment of CCR4-NOT by GW182. The translational repression can also be induced by the miRISC by inhibiting a step after initiation, such as promoting ribosome drop-off or stimulating proteolysis of the nascent peptide.

In cancer, changes in specific oncogenic and tumor suppressor miRNAs, as well as alterations in the miRNA expression profile in human tumors, have been shown to play a key role in the development of cancer. For example, let-7 family of miRNAs is downregulated in several types of cancer and is associated with poor patient outcomes [70]. However, several studies have established that mature miRNA accumulation also occurs after transcription being involved mechanisms of posttranscriptional regulation [67]. Alterations in the miRNA biogenesis pathway can also have an important role in cancer progression. In fact, it has been reported that mutations in the miRNA-processing machinery and dysregulation of miRNA biogenesis pathway are implicated in the pathogenesis of human disorders, including cancer [11]. Moreover, the core biogenesis machinery components, including Drosha, DGCR8, DICER1, and TRBP, are subject to posttranslational control such as phosphorylation and/or acetylation (reviewed in [10]). All of these dysfunctions in components of the miRNA biogenesis pathway produce expression changes in a large number of cellular miRNAs which can be correlated with poor patient outcomes [71-73].

Several genetic defects in components of the miRNAprocessing machinery have been reported in cancer. Mutations in genes that encode DROSHA, DGRC8, TARBP2, 

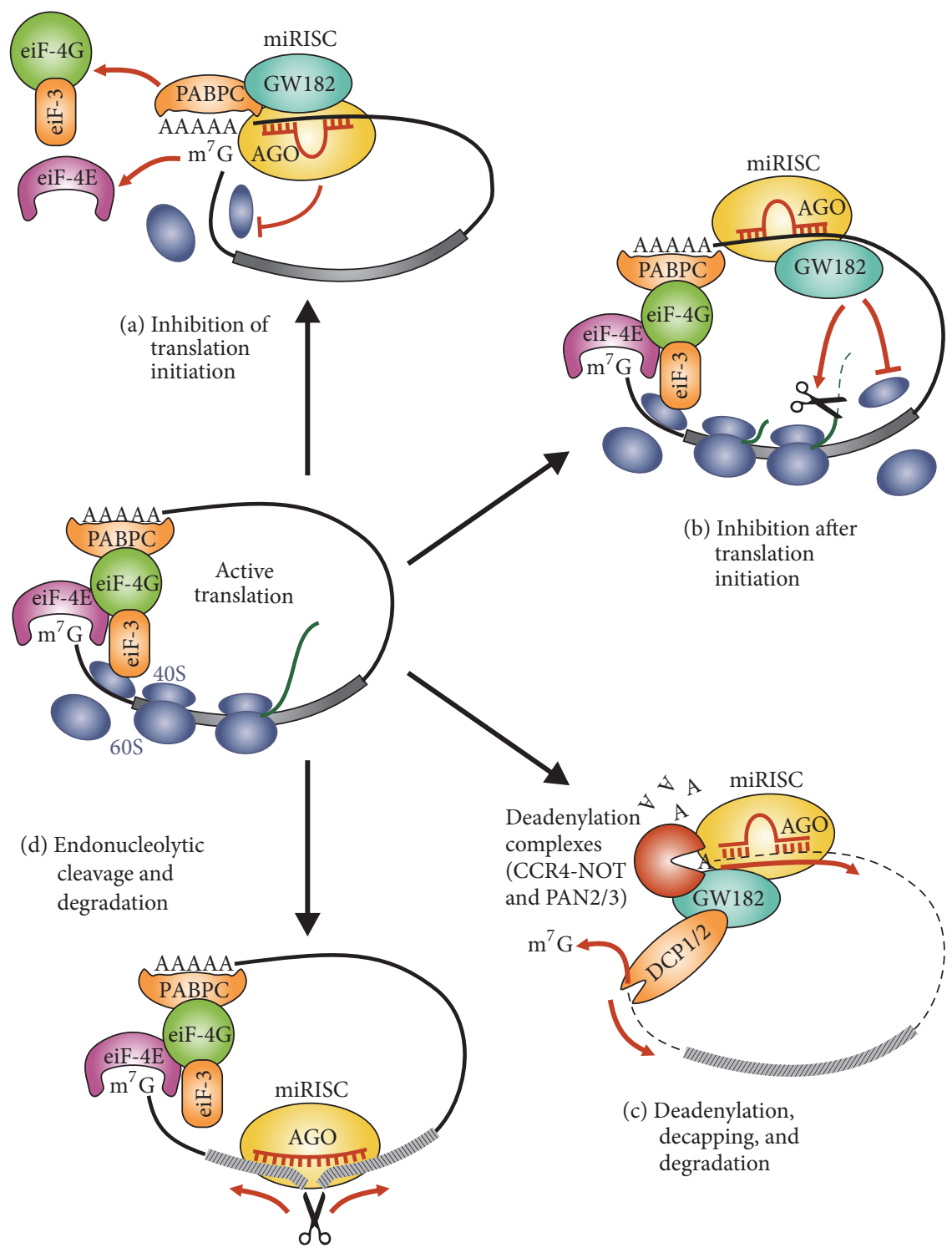

(b) Inhibition after translation initiation

FIGURE 4: Mechanisms of target regulation by miRNAs. miRNAs regulate gene expression through multiple pathways. Eukaryotic initiation factors bind the $5^{\prime}$ cap and the cytoplasmic poly(A)-binding protein (PABPC), connecting the $5^{\prime}$ and $3^{\prime}$ ends of mRNAs and stimulating their translation by the ribosome. (a) The miRNA-induced silencing complex (miRISC) can induce translational repression by blocking initiation; GW182 competes with eIF-4G in association with PABPC; and Argonaute (AGO) binds to the mRNA cap releasing eIF-4E, thus preventing the circularization required for efficient translation and the binding of ribosome $40 \mathrm{~S}$ subunit to the mRNA. (b) Translational repression can also be induced by the miRISC by inhibiting a step after initiation, such as promoting ribosome drop-off or stimulating proteolysis of the nascent peptide. (c) Partial pairing of the miRNA complex to target $3^{\prime}$ UTR sites can result in deadenylation of the mRNA by the CCR4-NOT or the PAN2/3 complexes and decapping by DCP1/2 (all of them recruited by GW182). Loss of the poly(A) tail causes dissociation of PABPC and leads to degradation of the mRNA. (d) Finally, perfect pairing between a miRNA and its target site induces endonucleolytic cleavage by AGO, leading to rapid degradation of the mRNA (occurs mainly in plants) [15].

DICER1, and XPO5 (exportin 5) point out the relevance of the miRNA biogenesis pathways in cellular transformation $[9,11,13,14,74,75]$. For example, the export of pre-miRNAs into the cytoplasm is mediated by the exportin 5/RanGTP complex. XPO5-inactivating mutations were identified in sporadic colon, gastric, and endometrial tumors with microsatellite instability. These XPO5 mutations impair premiRNA export to the cytoplasm and result in an accumulation of pre-miRNAs in the nucleus, leading to defects in miRNA biogenesis [75].
Alterations in the expression of components of the miRNA machinery are also involved in CRC. Kim and coworkers [76] analyzed the mRNA expressions of DGCR8 and $\mathrm{AGO} 2$ in 60 CRC tissues and adjacent histologically nonneoplastic tissues by using quantitative real-time PCR. They found that whereas mRNA expression level of DGCR8 is upregulated in CRC, the expression of AGO2 mRNA was not significantly altered in CRC tissues. Other studies using 237 samples from colorectal adenocarcinomas with moderate differentiation showed that a direct correlation between Dicer 
upregulation and poor prognosis in patients with CRC as also occurs on prostate cancer. On the contrary, in breast, lung, and ovary cancer, Dicer has been shown to be a marker of good prognosis [77]. It has also been reported that an increased expression of Dicer mRNA in normal mucosa from CRC patients is significantly related to poor survival independently of gender, age, tumor site, stage, and differentiation [78]. Finally, a study on the expression of Drosha, Dicer, and Ago $2 \mathrm{mRNAs}$ and protein in three colon cancer cell lines and in human CRC samples revealed that they were present in all the samples analyzed and the authors suggested that they were possibly implicated in CRC pathobiology. mRNA levels of Dicer were significantly augmented in stage III compared to stage II tumors suggesting that Dicer might have a role in the progression of these tumors to advanced stages [79].

\section{Noncoding RNAs and Their Involvement in Colorectal Cancer}

5.1. MicroRNAs and Colorectal Cancer. miRNAs are critical regulators of gene expression and an altered expression of miRNAs has been shown to be associated with various types of cancer. Figure 1(a) shows the increasing number of publications dealing with the relationship between miRNAs and cancer. More than 50\% of human miRNA genes are often located in specific chromosomal regions prone to damage through deletion, amplification, or translocation, which may result in malignant transformation [7]. Cancer development can also arise from dysregulation of miRNA biogenesis pathway [74]. In the context of cancer, miRNAs may have oncogenic or tumor-suppressive roles according to the effect they have on pathways leading to tumor development. Oncogenic miRNAs, or oncomiRs, target and downregulate endogenous tumor suppressor genes, whereas tumor suppressor miRNAs play an important role in downregulating genes associated with growth and metastasis. Thus, overexpression, genetic amplification, and gain-of-function mutations of oncogenic miRNAs as well as genetic deletion and loss-of-function mutations of tumor suppressor miRNAs are linked to human cancer. Furthermore, global miRNA depletion caused by genetic and epigenetic alterations, dysregulation of components of the miRNA biogenesis machinery, or changes in global miRNA levels resulting from a defective miRNA biogenesis pathway play critical roles in the pathogenesis of human disorders, including cancer $[11,74,220]$.

In CRC, some miRNAs are abnormally downregulated or upregulated; thus they may act as tumor suppressor or as oncogenes in tumor development. In addition, miRNA expression patterns have been suggested as predictive prognosis markers in CRC and support diagnosis of poorly differentiated tumors. The involvement of oncogenic and tumor suppressor miRNAs in key signaling pathways in CRC, their inductors, and targets, as well as the changes in the expression of some of them in the transition from normal colon mucosaadenoma-carcinoma-advanced carcinoma, have been also described in many different review articles [47, 221-230]. An overview of some oncogenic and tumor suppressor miRNAs involved in CRC, their role in CRC, and the verified targets is shown in Table 2.
Accumulating evidence strongly indicates that aberrant miRNA expression is an important feature of CRC. The first association between miRNAs and CRC was described by Michael and coworkers in 2003 [87], using CRC tissue compared to healthy tissue (Table 1). Several miRNA sequences were identified, among them miR-21, miR-143, miR-145, and miR-200c. miR-143 and miR-145 exhibited significantly reduced levels of the fully processed miRNA in tumors compared to normal specimens. On the other hand, the first study that evaluated the association between miRNA expression patterns and CRC prognosis or therapeutic outcome was carried out by Schetter and coworkers [231]. miRNA microarray expression profiling of tumors and paired nontumor tissues was performed on a US test cohort of 84 patients with CRC, evaluating associations with tumor status, TNM staging, survival prognosis, and response to adjuvant chemotherapy. Associations were validated in a second, independent Chinese cohort of 113 patients using quantitative reverse transcription PCR assays. They identified 5 miRNAs which were significantly altered in CRC. In particular, miR21 was overexpressed in $87 \%$ of colon cancers specimens and higher miR-21 expression correlated with poorer outcome.

Several studies have described the differentially expressed miRNAs in CRC. Wu and coworkers [232] measured the differential expression of miRNAs in colorectal adenocarcinoma tissues from 28 patients and analyzed their profiles at various differentiation stages. This study compared the expression level of 1547 miRNAs using qRT-PCR. Among them, 93 were found to be significantly dysregulated in colorectal adenocarcinoma relative to normal tissues. In particular, miR-1, miR-145, and miR-145* were downregulated more than tenfold and were suggested as potential biomarkers for CRC diagnosis. Furthermore, 58 miRNAs demonstrated significantly altered expression between well and moderately differentiated cancers, and 32 could be used to distinguish normal from cancerous tissues, as well as different levels of differentiation. In other study, microarrays were used to profile the expression of 315 human miRNAs in 10 normal mucosa samples and 49 stage II colon cancers differing with regard to microsatellite status and recurrence of disease. Several miRNAs were differentially expressed between normal tissue and tumor microsatellite subtypes, with miR-145 showing the lowest expression in cancer relative to normal tissue. Functional studies also showed that miR-145 potently suppressed growth of different colon carcinoma cell lines (LS174T, HCT116, and DLD1 cells) [233].

Hamfjord and coworkers [234] performed a global analysis of dysregulated miRNAs in paired samples of normal mucosa and tumor from eight patients with CRC. In this study, the normal and adjacent tumor tissue samples were paired, thus taking into account the baseline differences between individuals when testing for differential expression. At least, 37 miRNAs were identified as differentially expressed between the matched pairs of CRC tissues and normal colon mucosa, 19 downregulated and 18 upregulated. Some of these miRNAs were previously published as potential regulators in colorectal adenocarcinomas, such as miR-1, miR-96, and miR-145. They discovered 16 dysregulated miRNAs, which were not previously associated with colorectal carcinogenesis. 
TABLE 2: Oncogenic and tumor suppressor miRNAs involved in colorectal cancer.

\begin{tabular}{|c|c|c|c|}
\hline miRNA & Verified targets & Role in colorectal cancer & References \\
\hline \multicolumn{4}{|c|}{ Oncogenic miRNAs (upregulated) } \\
\hline miR-18a & ATM & $\begin{array}{l}\text { Blocking apoptosis, DNA repair, and sensitivity to } \\
\text { etoposide }\end{array}$ & {$[114]$} \\
\hline $\operatorname{miR}-21$ & PTEN, PCDC4, TGFBR2, CDC25A & $\begin{array}{c}\text { Proliferation, apoptosis, invasion, migration, CSC } \\
\text { maintenance }\end{array}$ & {$[115-117]$} \\
\hline miR-29a & KLF4 & Invasion, metastasis & {$[118]$} \\
\hline miR-31 & HIF1A, RhoBTB1, RASA1 & Proliferation, migration, invasion, tumor growth & [119-121] \\
\hline miR-32 & PTEN & Proliferation, migration, invasion, apoptosis & [122] \\
\hline miR-92a & PTEN & Proliferation, migration, invasion, apoptosis, EMT & [123] \\
\hline miR-95 & SNX1 & Proliferation, tumor growth & [124] \\
\hline miR-96 & TP53INP1, FOXO1, FOXO3A & Proliferation & [125] \\
\hline miR-103 & DICER, PTEN & Proliferation, migration, tumor growth & [126] \\
\hline $\mathrm{miR}-135 \mathrm{a} / \mathrm{b}$ & APC & Proliferation & [127] \\
\hline miR-155 & MLH1, MSH2, MSH6 & Altering DNA damage response & {$[128]$} \\
\hline miR-181a & WIF-1, PTEN & $\begin{array}{l}\text { Proliferation, migration, invasion, tumor growth, liver } \\
\text { metastasis, metabolic shift, EMT }\end{array}$ & {$[129,130]$} \\
\hline $\operatorname{miR}-182$ & FOXF2 & Cell growth, invasion, increased $\beta$-catenin activity & {$[131]$} \\
\hline miR-196b & FAS & Blocking apoptosis & {$[132]$} \\
\hline miR-214 & PTEN, PDLIM2 & Inflammation & [133] \\
\hline $\operatorname{miR}-223$ & RASA1 & Tumor growth & {$[134]$} \\
\hline miR-224 & SMAD4 & Metastasis & [135] \\
\hline \multicolumn{4}{|c|}{ Tumor suppressor miRNAs (downregulated) } \\
\hline let-7 & KRAS & Proliferation & {$[91]$} \\
\hline miR-7 & EGFR, RAF1 & Proliferation & {$[136]$} \\
\hline miR-18a* & KRAS & Proliferation & {$[137]$} \\
\hline miR-26b & TAF12, PTP4A1, CHFR, ALS2CR2 & Proliferation, apoptosis, invasion, migration & {$[138]$} \\
\hline $\operatorname{miR}-27 b$ & VEDFC & Proliferation, angiogenesis & {$[139]$} \\
\hline miR-34a & SIRT1 & Apoptosis & [140] \\
\hline miR-100 & RAP1B & Proliferation, invasion, apoptosis & {$[141]$} \\
\hline miR-101 & SPHK1 & Angiogenesis & {$[142]$} \\
\hline miR-124 & STAT3 & Proliferation, apoptosis, tumor growth, differentiation & [143] \\
\hline $\operatorname{miR}-126$ & VEGFA, IRS-1, CXCR4 & $\begin{array}{c}\text { Proliferation, migration, invasion, cell cycle arrest, } \\
\text { angiogenesis }\end{array}$ & {$[144-146]$} \\
\hline miR-133a & FSCN1, LASP1 & $\begin{array}{l}\text { Proliferation, invasion, migration, tumor growth, } \\
\text { metastasis, phosphorylation of ERK/MEK }\end{array}$ & {$[147,148]$} \\
\hline miR-133b & TBPL1, CXCR4 & Proliferation, invasion, migration, apoptosis & {$[149,150]$} \\
\hline miR-139 & IGF1R, NOTCH1 & $\begin{array}{l}\text { Proliferation, migration, invasion, apoptosis, tumor } \\
\text { growth, cell cycle arrest }\end{array}$ & {$[151,152]$} \\
\hline miR-143 & ERK5, KRAS, IGF1R & Proliferation & {$[153,154]$} \\
\hline miR-144 & MTOR & Proliferation & [155] \\
\hline miR-145 & IRS1, NRAS, IGF1R & $\begin{array}{c}\text { Proliferation, invasion, migration, angiogenesis, tumor } \\
\text { growth, metastasis }\end{array}$ & {$[153,154,156]$} \\
\hline miR-148b & CCK2R, PIK3R3 & Proliferation, tumor growth, tumor size & {$[157,158]$} \\
\hline miR-194 & PDK1, AKT2, XIAP, MAP4K4 & $\begin{array}{l}\text { Proliferation, apoptosis, migration, angiogenesis, cell } \\
\text { cycle arrest, tumor growth and size, differentiation, } \\
\text { metastasis }\end{array}$ & {$[159,160]$} \\
\hline
\end{tabular}


TABLE 2: Continued.

\begin{tabular}{|c|c|c|c|}
\hline miRNA & Verified targets & Role in colorectal cancer & References \\
\hline miR-195 & BCL2 & Apoptosis & {$[161]$} \\
\hline $\mathrm{miR}-200 \mathrm{a} / \mathrm{c}$ & ZEB1/2 & EMT & {$[87,162]$} \\
\hline miR-206 & NOTCH3 & Proliferation, migration, apoptosis, cell cycle arrest & {$[163]$} \\
\hline $\operatorname{miR}-214$ & FGFR1 & $\begin{array}{c}\text { Proliferation, migration, invasion, tumor growth, } \\
\text { metastasis }\end{array}$ & {$[164]$} \\
\hline miR-218 & BMI1 & Proliferation, apoptosis, cell cycle arrest & {$[165]$} \\
\hline $\operatorname{miR}-224$ & $\mathrm{CDC} 42$ & Migration & {$[166]$} \\
\hline miR-320a & CTNNB1, RAC1 & Proliferation, migration, invasion, cell cycle arrest & {$[167,168]$} \\
\hline miR-342 & DNMT1 & $\begin{array}{l}\text { Proliferation, invasion, cell cycle arrest, tumor growth, } \\
\text { metastasis }\end{array}$ & {$[169]$} \\
\hline miR-365 & BCL2, CCND1 & Apoptosis & [170] \\
\hline miR-375 & PIK3CA & Proliferation, cell cycle arrest, tumor growth & {$[171]$} \\
\hline miR-378 & VIM & $\begin{array}{l}\text { Proliferation, invasion, tumor growth and size, } \\
\text { metastasis }\end{array}$ & {$[172]$} \\
\hline $\operatorname{miR}-429$ & ONECUT2 & Migration, invasion, EMT & [173] \\
\hline $\operatorname{miR}-455$ & RAF1 & Proliferation, invasion & [174] \\
\hline miR-491 & BCLXL & Apoptosis & [175] \\
\hline miR-638 & SOX2 & Invasion, migration, EMT & [176] \\
\hline
\end{tabular}

The downregulated ones were miR-490-3p, miR-628-3p and miR-628-5p, miR-1297, miR-3151, miR-3163, miR-3622a-5p, and miR-3656 and the upregulated miR-105, miR-549, miR1269, miR-1827, miR-3144-3p, miR-3177, miR-3180-3p, and miR-4326. On the other hand, a gene module-based approach to inferring key miRNAs regulating the major gene network alterations in CRC has been proposed comparing 90 normal and 107 CRC samples in total. Among the inferred candidates, three miRNAs, miR-101, miR-124, and miR-139, are frequently downregulated in CRC tumors. Computational and experimental analyses demonstrate that miR-139 can inhibit cell proliferation and cell cycle G1/S transition. In addition, miR139 was found to be significantly downregulated in early pathological cancer stages and its expression remained at very low levels in advanced stages. MiR-139 was determined to be a key tumor suppressor in early cancer development [235]. Dysregulation in circulating blood miRNAs is reflective of those in colorectal tissues; in fact, a triple miRNA classifier consisting in miR-193a-3p, miR-23a, and miR-338-5p has been suggested as a potential blood biomarker for early detection of CRC [236].

5.2. Signaling Pathways in Colorectal Cancer Regulated by miRNAs. Considering the classic multistep colorectal carcinogenesis model proposed by Fearon and Vogelstein in 1990 [20], most CRCs progress through the sequential accumulation of molecular alterations associated with the adenomato-carcinoma progression. Dysregulation of a significant number of miRNAs in CRC and colon CSCs has been described in the literature $[47,221,225,237,238]$ but special attention has been directed towards those miRNAs involved in signaling pathways and cellular processes. Among the signaling pathways that are modified in CRC carcinogenesis are those that result in activation of prosurvival, proproliferative, and metastasis (i.e., Wnt, EGFR, and TGF- $\beta$ ) as well as impairment of p53 function [34, 226]. Extensive studies evidence that miRNAs can regulate all the major pathways in CRC; briefly, this includes their impact on $\beta$ catenin/Wnt signaling (miR-135a/b, miR-139, miR-145, miR17-92), proliferation (let-7 family, miR-18a, miR-21, miR-126, miR-143, miR-200c), apoptosis (miR-34a, miR-133b, miR195), cell cycle control (miR-34a, miR-192, miR-215, miR675), p53 signaling (miR-34b/c), differentiation (miR-141, miR-200c), and migration and invasion (miR-126, miR-143, miR-196a, miR-200a/b/c, miR-373, miR-520c) [34].

Wnt activation signaling pathway is crucial for the regulation of stem cell activity to the intestinal crypt base and for the renewal of the epithelial cells. Activation of Wnt signaling induces cell survival and inhibits cell death and differentiation. The mutation of the tumor suppressor APC is one of the first events for the initiation of colorectal neoplasia. A miRNA-mediated mechanism has been described in CRC to control the expression of the APC gene and consequent activation of the Wnt signaling pathway. In adenocarcinomas, as well as premalignant colorectal adenomas, oncomiRs miR$135 \mathrm{a}$ and miR-135b are overexpressed and directly target the $3^{\prime}$ UTR of the APC mRNA, suppress the APC expression, and activate Wnt signaling [127]. In addition, several Wntpathway genes contain binding sites in the $3^{\prime}$ UTR of their corresponding mRNAs for members of the miR-34 family. The miR-34 family comprises three processed miRNAs that are encoded by two different genetic loci. MiR-34a is encoded by one transcript, and miR-34b and miR-34c arise from a common primary transcript. The members of the miR34 family are direct p53 targets, which induce apoptosis, cell cycle arrest, and senescence [239]. $\beta$-Catenin binds to 
members of the TCF/LEF family of transcription factors whose activity is mainly regulated by the miR-34 family. In this way, miR-34 represses the activity of the TCF/LEF complex and establishes a relationship between the Wnt signaling pathway and p53 activity. Loss of p53 functions causes increased activity of Wnt signaling cascade and promotes the Snail-dependent EMT. Moreover, higher expression of oncogenic miR-21 in adenomas and CRC relative to normal tissues suggests that abnormal expression of this miRNA is an early event in the progression towards CRC. It has also been reported that miR-21 promotes cell migration and invasion by targeting the PDCD4 (Programmed Cell Death Protein 4) and PTEN (phosphatase and tensin homolog) tumor suppressor genes $[115,116]$.

In early stages of cell transformation in CRC, an upregulation of miR-17-92 cluster has been detected. PTEN and E2Fs mRNAs were among the first validated miR-17-92 targets. Recently, Li and coworkers [240] have demonstrated a direct link between APC and the miR-17-92 cluster. APC represses miR-17-92 through inhibition of $\beta$-catenin. Mutation of APC leads to stabilization of $\beta$-catenin, which in turn binds to and activates the miR-17-92 promoter. Moreover, elevated level of $\beta$-catenin is significantly correlated with miR-19a (that belongs to the miR-17-92 family) overexpression in CRC.

miRNAs have been reported to regulate the stemness of colon CSCs mainly via regulation of important signaling pathways as Wnt/ $\beta$-catenin and Notch, acting also on processes that involve CSCs altering the expression of cell cycle and EMT-related genes. Notch and Wnt pathways are involved in crypt development and in proliferation and selfrenewal of colon SCs, decreasing apoptosis; these pathways are commonly upregulated in SC populations of colon cancer tissue. The most frequently reported miRNAs involved in the regulation of colon CSCs are miR-21 and miR-34a through modifications in the Wnt/ $\beta$-catenin and Notch/Ckit-Erk pathways, respectively. miRNA-21 is overexpressed in colon CSCs and is able to downregulate the expression of TGFBR2 gene (TGF- $\beta$ receptor II), resulting in activation of the $\mathrm{Wnt} / \beta$-catenin pathway and consequent increase in the expression of downstream target genes as $c-M y c$ and cyclinD1 [117]. miR-21 also targets PTEN reducing its expression and with a concomitant activation of Akt signaling pathway, thus increasing the tumorigenic properties of colonic CSCs [241]. Finally, miR-21 can also control the expression of other miRNA, miR-145 and, in turn, increase the expression and activity of intact K-Ras in colon CSCs [242]. miR-34a is downregulated in colonic CSCs and restoration of its expression leads to differentiation of CSCs derived from colon cancer tissue to non-CSCs. miR-34a targets Notch signaling pathway, that plays an important role in symmetrical and asymmetrical cell division of stem cells. Moreover, c-kit (stem cell factor receptor) is a direct mRNA target of miR-34a; thus, downregulation of miR-34a leads to overexpression of c-kit and activation of several stemness markers [238].

The epidermal growth factor receptor (EGFR) signaling pathway is also involved in the development of CRC. EGFR can activate different signal transduction pathways as Ras/MAPK and the phosphatidylinositide 3 kinase- (PI3K) Akt pathways. Activating K-Ras mutations have been described to induce activation of various downstream effectors that mediate tumor growth, survival, and metastasis in many cancers $[34,226]$. In CRC, tumor suppressors, miR143 and miR-145, that are located on chromosome 5, are reduced. Downregulated expression of miR-143 and miR145 has been described to target multiple mRNAs related to the MAPK signaling pathway, including $K$-Ras, ERK5, IRS1 (insulin receptor substrate 1), all of them involved in the transition of an early adenoma to advanced stages $[153,226]$. Furthermore, PTEN, a dominant negative regulator of the Akt, has been shown to be the target of different miRNAs, such as miR-21, miR-32, miR-92a, and miR-181a (Table 2). Other signaling pathways involve TGF- $\beta$ family that regulates cellular processes such as proliferation, differentiation, apoptosis, and migration. Several miRNAs have been described to regulate TGFBR2, such as miR-17-5p, miR-20a, miR-21, miR23b, miR-106a, and miR-301a. Particularly, miR-21, activated by the Wnt signaling pathway, is involved in stemness by regulating TGFBR2 signaling [243]. The oncogenic miR-1792 cluster, that is modulated by $\mathrm{c}-\mathrm{Myc}$, represses TGF- $\beta$ responses by silencing TGFBR2 and SMAD4.

The tumor suppressor p53 is also involved in CRC by modulation of the response to different stress signals and controlling processes as senescence, cell cycle arrest, apoptosis, invasion, and metastasis [221, 226, 239]. Bioinformatics sequence analyses propose that up to $46 \%$ of the miRNA potential promoters contain a p53-binding site [215]. Several miRNAs are related to p53, among them are the following: let-7i, miR-20a, miR-21, miR-25, miR-34a/b/c, miR-145, miR181b, miR-183, miR-195, miR-215, and miR-451. Interestingly, the miR-34 family is p53-inducible; miR-34a is upregulated in CRC patients. A positive feedback loop between miR-34a and p53 has been proposed as miR-34a activates p53 by targeting sirtuin mRNA, a key regulator of p53 activity. Moreover, miR34a downregulates transcription factor E2F and upregulates p53 in several cancers such as CRC [221].

EMT, that plays an important role in tumor progression, invasiveness, and therapeutic failure, is also regulated by Wnt and Notch signaling pathways through increasing levels of $\beta$-catenin that translocates to the nucleus and ultimately induces specific genes essential for EMT triggering by Wnt or by activation of NF- $\kappa \mathrm{B}$ or TGF- $\beta$ pathways by Notch. Several EMT-associated miRNAs are involved in CRC such as the p53 responsive miR-200 family (miR-200a/b/c, miR141, and miR-429), whose downregulation is believed to be an essential feature of EMT. In addition, miR-146a and miR203 are also involved in colon CSC regulation via EMT signaling pathways. The first one is upregulated by the EMT inducer Snail that causes the opposite effect on miR-203. miR-200a and miR-200c are also downregulated in CSCs, allowing the expression of EMT inducers Zeb1 and Zeb2 with a concomitant decrease in E-cadherin levels and increased expression of stem cell markers CD166 and CD133 [162]. Other miRNAs have been described as important for the maintenance of the cancer stem cell phenotype. miR-215 and miR-140 are involved in chemoresistance to methotrexate and 5-FU treatments and in differentiation of CSCs derived from HCT-116 cells $\left(\mathrm{CD} 133^{+}, \mathrm{CD} 44^{+}\right)$possibly by targeting cell cycle and differentiation related genes [183, 185, 238]. 
Similarly, a population of colon CSCs expressing a CD133 surface phenotype from human HT29 colonic adenocarcinoma cells showed overexpression of 11 and downregulation of 8 miRNAs which could be potentially involved in the regulation of stem cell differentiation [244].

As mentioned earlier, the miR-34 family is p53-inducible and miR-34a is upregulated in CRC patients. However, miR$34 \mathrm{~b} / \mathrm{c}$ expression is not detectable in several CRC cell lines probably due to an epigenetic silencing of these miRNAs [245]. Finally, miRNAs also are regulators of the cancer epigenome; DNMTs, HDACs, and HMTs are targets of miRNAs [246]. DNMT3a is downregulated in CRC and is the target of miR-143. Similarly, miR-342 and miR-185 interact with the $3^{\prime}$ UTR of DNMT1 mRNA [169]. Another example is miR-140; its overexpression inhibits cell proliferation of colon cancer HCT 116 (wt-p53) cells but presents lower effect in HCT 116 (null-p53) cells. This miRNA induces p53 and p21 expression and produces cell cycle arrest only in cells containing wild type of p53. Histone deacetylase 4 (HDAC4) was confirmed to be one of the important targets of miR140. In addition, the expression of endogenous miR-140 was significantly elevated in CD133(+hi)CD44(+hi) colon cancer stem-like cells that exhibit slow proliferating rate and chemoresistance [183].

5.3. MicroRNA Polymorphisms and CRC Risk. Single nucleotide polymorphisms (SNPs) are common variants in the human genome and have been reported to influence disease susceptibility. SNPs in miRNAs and related loci are often located in pri-miRNA and pre-miRNA sequences, seed sequences, and the $3^{\prime}$ UTR regions of target mRNAs [247]. Thus, such polymorphisms might influence miRNA function in three ways: by altering transcription of the primary transcript, affecting pri- and pre-miRNA processing, and modifying miRNA-mRNA interactions [248]. SNPs in miRNAs may ultimately result in the alteration of their expression and/or maturation, with possible consequences for cancer development and progression. Furthermore, approximately half of miRNA genes are located in cancer-related regions [249]. Thus, variations in these sequences may result in significant functional consequences, making them ideal candidates for cancer risk prediction.

It has been reported that around 35\% of analyzed CCRs are due to genetic or hereditary factors. These factors may arise from single nucleotide polymorphisms (SNPs) or additional genetic abnormalities in both coding and noncoding genes [237]. Taking into account the important role of miRNAs in the initiation and development of CRC, the occurrence of SNPs in these molecules has been investigated as they may influence mRNA function or interrupt miRNA expression, altering its sequence, the binding site to mRNA, or even its processing potentially contributing to cancer susceptibility. Therefore, miRNA polymorphisms may be used as specific markers of predisposition for CRC diagnosis and prevention.

SNPs in specific miRNAs may alter the susceptibility of a patient to develop CRC $[248,250]$. SNPs have been detected in association with an increased risk of CRC affecting either miRNAs (SNPs in miR-196a, miR-149, and pre-miR-27a or in miR-257a, affecting binding to $3^{\prime} \mathrm{UTR}$ of MBL2 mRNA) [251-253], or the $3^{\prime}$ UTR of an mRNA, as in CD86, that alters binding of several miRNAs (in this case, miR-184, miR200a, miR-212a, miR-337, and miR-582) [254], or DOK3, affecting binding of miR-370 [255]. It has also been observed that specific SNPs of miRNA-processing machinery genes (as DICER or GEMIN3) may affect CRC susceptibility [256]. In addition, SNPs in miRNAs have been associated with CRC survival and recurrence, and thus they might be useful in predicting therapy response. This is the case of two SNPs (rs4919510 in miR-608 and rs213210 in miR-219-1) that were genotyped in 1083 CRC patients to evaluate their effect on clinical outcomes. Carriers of the variant T allele in rs213210 and receiving 5-FU chemotherapy were associated with a significantly worse survival and an increased risk of relapse, whereas patients carrying the G allele of rs4919510 and undergoing adjuvant chemotherapy were at decreased risk of relapse [257].

5.4. Long Noncoding RNAs in Colorectal Cancer and Regulation of miRNA Function. The long noncoding RNAs (lncRNAs) are mainly RNA polymerase II transcripts usually longer than 200 nucleotides. Some lncRNAs show additional similarities to mRNA, such as a $5^{\prime}$ cap, $3^{\prime}$ poly(A) tail, even though they lack an open reading frame and have no potential to encode protein. The number of genes contained within the gene family of lncRNAs is 214 in humans [http://www .genenames.org/cgi-bin/genefamilies/set/788]. lncRNA may be located in the nucleus, chromatin, or cytoplasm; these different localizations point out to different biological functions. Thus, lncRNAs regulate a variety of key cellular processes such as epigenetic silencing, gene transcription and translation, cell cycle and apoptosis, and cell differentiation and proliferation $[9,53]$. For example, lncRNAs as Xist (X inactive-specific transcript, related to X-chromosome silencing) and HOTAIR (HOX Transcript Antisense Intergenic RNA) interact with chromatin remodeling complexes to induce changes in chromatin packaging, leading to reduced target gene expression [258]. In fact, HOTAIR was the first identified lncRNA that plays a critical oncogenic role through epigenetic regulatory mechanisms [259]. IncRNAs can also act as coactivators of transcription factors by interacting with RNA-binding proteins, and this interaction alters the localization and activity of the proteins. Additionally, lncRNA transcripts could competitively inhibit the ability of miRNAs to interact with their mRNA targets. They act as miRNA sponges sequestering miRNAs analogous to how artificial miRNA sponges function (Figure 3) [103, 260]. However, although empirical evidence supporting the hypothesis that competitive endogenous RNAs (ceRNAs) as lncRNAs, circRNAs, or pseudogenes can act as miRNA sponges, recent studies that model transcriptome-wide binding-site abundance suggest that physiological changes in expression of most individual transcripts will not compromise miRNA activity [261].

The number of research papers and reviews dealing with lncRNAs and their involvement in cancer is exponentially increasing every year. Figure 1(a) (inset) shows the evolution of the number of papers along the last years. Several reviews 
recapitulate the knowledge in this area $[9,46,47,53,262-$ 264]. Here we describe only the function of a reduced number of lncRNAs and mainly those that are involved in CRC.

lncRNAs exhibit unique profiles in various human cancers, reflecting disease progression and serving as a predictor of patient outcome [265]. Their roles as drivers of tumor suppressors and oncogenic function have been described in different types of cancer [266]. Several studies have highlighted the role of lncRNAs in the development of CRC and their involvement in chemoresistance of CRC cells [264,267]. They are involved in processes related to CRC progression through stimulating or inhibiting cell proliferation, apoptosis, differentiation, invasion, and metastasis. CRC-related lncRNAs have been described to regulate gene expression by a broad range of mechanisms [267] such as (i) by epigenetic modifications (DNA methylation, histone modification, chromosomal instability, X-chromosome inactivation, and genomic imprinting), (ii) by lncRNA-miRNA interactions, (iii) by their actions as small RNAs or miRNA precursors or pseudogenes, (iv) by lncRNA-protein interactions, and (v) through their function as structural RNAs, in scaffolding ribonuclear protein complexes. In addition, as some lncRNAs have been detected in human body fluids by PCR, such as in plasma and urine, they have also been suggested as novel potential biomarkers for CRC diagnosis and prognosis as well as in the prediction of the response to therapy [268].

Interactions between lncRNAs and RNA sequences have been described as a regulatory posttranslational mechanism. In this way, lncRNAs can function as competing endogenous RNAs, miRNA sponges, or as pseudogenes to serve as decoys for miRNAs. As a result, alterations in target genes or in the biological function of the miRNAs could be expected. This mechanism of inhibition of miRNA activity was discovered in 2007 studying the phosphate homeostasis in Arabidopsis thaliana (Table 1). The non-protein-coding RNA IPS1 (Induced by Phosphate Starvation 1) contains a motif with sequence complementarity to miR-399, the phosphate starvation-induced miRNA. However, the pairing is interrupted by a mismatched loop at the expected miRNA cleavage site in IPS1. Thus, IPS1 RNA is not cleaved but instead sequesters miR-399 [104]. In mammalian cells, Ebert and coworkers established the name "miRNA sponges" describing the specific competitive inhibitors from transcripts expressed from strong promoters which contained multiple tandem binding sites to several miRNA seed families [103]. In this way, as we comment later on, several CRCrelated lncRNAs may also regulate gene expression in CRC not only by binding to target proteins but also by binding to miRNAs and, consequently, preventing specific miRNAs from binding to their target mRNAs.

A clear example of miRNA sponge is the lncRNA HULC (Highly Upregulated in Liver Cancer) that is located on the human chromosome $6 \mathrm{p} 24.3$. It is formed by two exons and a $500 \mathrm{bp}$ intron and contains a $3^{\prime}$ poly(A) tail and a conserved miR-372 target site [269]. HULC binds to its target miR-372 and acts as a sponge inhibiting the binding of miR-372 to the mRNA transcript. HULC is overexpressed not only in hepatocellular carcinoma but also in hepatic CRC metastasis whereas, in normal tissues, primary CRC, or those cancers that metastasize to lymph nodes, its expression is null [270, 271]. The involvement of HULC in CRC metastasis to the liver suggests its role as a new biomarker for the evaluation of hepatic metastasis potential of CRC.

Loc285194 (or LSAMP antisense RNA 3) is a lncRNA with more than $2 \mathrm{~kb}$ in length, which contains 4 exons and is present in the focal region of chr3q13.31 (osteo3q13.31), which is the most altered region in osteosarcomas. It has two binding sites for miR-211 in its exon 4 and acts as a potential tumor suppressor as deduced from knockdown experiments, which showed an increased cell proliferation. It has been described that loc285194 is downregulated in colon tumor specimens compared with the normal ones and is a direct transcriptional target of $\mathrm{p} 53$ through the negative regulation of miR-211 [264, 272]. Interestingly, miR-211 promotes cell growth and represses loc285194 expression, thus creating a reciprocal repression feedback loop.

In addition to binding miRNA, several studies have demonstrated that lncRNAs can regulate gene expression by acting as miRNA precursors. In this way, lncRNA H19 (located in chromosome 11q15.5 and $6295 \mathrm{nt}$ in length) has different roles in CRC. It functions as miRNA sponge, as a miRNA precursor, and was also described as an epigenetic regulator [264]. Thus, it is a good potential target for CRC treatment. $\mathrm{H} 19$ can promote EMT progression and accelerate tumor growth by acting as competing endogenous RNA for miR-138 and miR-200a in CRC [273]. Moreover, H19 can be processed to yield the precursor of miR-675 [274] and its expression is positively correlated with the level of miR-675 that is involved in the downregulation of Rb expression [275]. The sequence of mature miR-675 aligns with a sequence of the $3^{\prime}$-UTR of Rb mRNA, and the level of Rb protein appears to be negatively correlated with the levels of both H19 and miR675 in human CRC cells [276].

The lncRNA MALAT1 (Metastasis-Associated Long Adenocarcinoma Transcript 1) is located in chromosome 11q13.1 and has a length of $8708 \mathrm{nt}$. It promotes cell proliferation, migration, tumor growth, and metastasis in CRC cells. MALAT1 interacts with and changes the distribution of splicing factors, such as SR (serine/arginine-rich) proteins. Depletion of MALAT1 affects the abundance, location, and activity of SR proteins and changes the alternative splicing of a series of pre-mRNAs [277].

lncRNAs can also function as diagnostic and prognostic biomarkers for CRC. CRNDE (Colorectal Neoplasia Differentially Expressed) and HOTAIR, for example, are upregulated in neoplastic tumor tissue and in the blood of CRC patients [278, 279]. The lncRNA CRNDE is highly elevated in $\mathrm{CRC}$, is transcribed from chromosome 16, and interacts with components of the polycomb repressive complex 2 (PRC2) and CoREST complexes [280]. In addition, HOTAIR is a lncRNA that plays a role as an oncogenic molecule. It is located on chromosome $12 \mathrm{q} 13.13$, has $2.2 \mathrm{~kb}$ of length and 6 exons, and has a $3^{\prime}$ poly(A) tail. Not only does HOTAIR interact with PRC2, but also it is necessary for PRC2 occupancy and histone $\mathrm{H} 3$ lysine-27 trimethylation of different genes. PRC2 is a histone methyltransferase that implements epigenetic silencing during different processes including cancer development [281]. Interestingly, unlike HOTAIR, 
the lncRNA PCAT-1 (Prostate Cancer-Associated ncRNA Transcript 1 , located in the chromosome 8q24) is repressed by PRC2. PCAT-1 is upregulated in CRC specimens compared with normal tissues. There is a positive correlation between PCAT-1 expression and distant metastasis of CRC. The overall survival rate of the patients with high expression of PCAT-1 is significantly lower than those with low expression [282].

The CCAT1 (Colon Cancer-Associated Transcript 1; located in chromosome 8q24.21, 2,628 nt in length) is highly expressed in CRC compared to normal tissue. CCAT1 upregulation is present in primary CRC tumors, including precancerous polyps, lymph nodes, blood, and distant CRC metastasis [283]. Its increased expression was also correlated with the clinical stage of the patients, lymph nodes metastasis, and survival time after surgery. CCAT1 functions as an oncogene; it is located close to the transcription factor $c$ $M y c$ gene and upregulates it, promoting tumor cell proliferation and migration. Moreover, c-Myc could induce CCAT1 transcription by directly binding to its promoter region. CCAT1 expression is upregulated in CRC cells and promotes cell proliferation and invasion. These findings suggest that c-Myc-activated lncRNA CCAT1 expression contributes to colon cancer tumorigenesis and metastatic process and could predict the clinical outcome of CRC and be a potential target for lncRNA direct therapy.

Interestingly, lncRNAs seem to be also involved in chemoresistance of CRC cells. In vitro studies revealed the downregulation of the lncRNA snaR (small NF90-associated RNA) and BACE1-AS (BACE1 antisense RNA) in 5-FU resistant CRC cells, contributing to increase viability and inhibiting apoptosis without altering the cell cycle [284]. Conversely, the overexpression of the lncRNA UCA1 (urothelial carcinoma associated 1) enhances cell proliferation and 5FU resistance in colorectal cancer by inhibiting miR-204-5p possibly acting as a sponge for this miRNA [285].

Finally, we will consider two lncRNA examples of molecules related to p53 and CRC. The lncRNA TP53TG1 (TP53 target 1) located in chromosome 7q21.12 is critical for the correct response of p53 to DNA damage and its expression is induced by p53 under conditions of cellular stress. DiazLagares and coworkers have described that TP53TG1 undergoes cancer-specific promoter hypermethylation-associated silencing. TP53TG1 binds to the multifaceted DNA/RNAbinding protein $\mathrm{YBX} 1$ to prevent its nuclear localization and thus the YBX1-mediated activation of oncogenes. TP53TG1 epigenetic inactivation in HCT-116 CRC cells releases the transcriptional repression of YBX1-targeted growthpromoting genes and creates a chemoresistant tumor [286]. The long intergenic noncoding RNA-p21 (lincRNA-p21; $3.1 \mathrm{~kb}, 2$-exon transcript, located near the $p 21$ gene) has a p53-binding motif in its promoter. It acts to repress many genes transcriptionally regulated by $\mathrm{p} 53$ and is aberrantly expressed in several types of cancer, including CRC. Experimental evidence using $30 \mathrm{CRC}$ tissues, their adjacent normal mucosa, and several CRC cell lines showed that lincRNA-p21 decreases which contributes to the elevation of $\beta$-catenin in CRC. It was also observed that the expression of lincRNAp21 increases following radiation exposure and enforced expression of the lincRNA enhances the CRC sensitivity to radiotherapy, by promoting cell apoptosis [287]. Considering the mechanism of gene regulation, the heterogeneous nuclear ribonucleoprotein K (hnRNP-K) associates with the promoters of many genes repressed by lincRNA-p21 in a lincRNA-p21 dependent manner. Dimitrova and coworkers [288] have described that lincRNA-p21 interacts with the hnRNP-K through its $5^{\prime}$ end and influences gene expression as a coactivator of $\mathrm{p} 21$ expression promoting the expression of polycomb target genes. After loss of lincRNA-p21, the expression of p21 decreased and, although hnRNP-K was present, the expression of the polycomb target genes was downregulated and the chromatin state of the genes was altered.

5.5. Circular RNAs in Colorectal Cancer and Their Role as miRNA Sponges. Circular RNAs (circRNAs) were reported more than 30 years ago by electron microscopy [289] and later on as several abnormally spliced transcripts in which exons from a candidate tumor suppressor gene (DCC) were scrambled during the splicing process in vivo [290]. However, they were considered as rare molecules that resulted from splicing artefacts or gene rearrangements. CircRNAs were rediscovered from RNA sequencing (2012/2013) and recent studies reporting high abundance, great diversity, and tissue and development specific expression indicate that they are not just a result of splicing errors. These studies have discovered thousands of endogenous circRNAs in mammalian cells, among them more than 2,000 circRNA in humans.

circRNAs are RNA molecules that form covalently closed continuous loops with joined $3^{\prime}$ and $5^{\prime}$ ends. They mainly arise from protein coding genes, from exons (exonic circRNA) or introns (intronic circRNA), by models of exon selfcircularization, by lariat-driven circularization, or by backsplice events [291, 292]. In this last process, the downstream exons ( $3^{\prime}$ splice donor site) are spliced to upstream exons ( $5^{\prime}$ splice acceptor site) in the primary transcript, yielding a circular transcript. Reverse complementary sequences or RNA-binding proteins are necessary for circRNA biogenesis. For intronic lariat-originated circRNAs, a consensus motif containing a seven-nucleotide GU-rich element at the $5^{\prime}$ splice site and an eleven-nucleotide C-rich element upstream the branchpoint has been reported [292].

A characteristic of circRNAs is their stability and resistance to RNase R. They are related to different cancers, including CRC, where they exhibit an aberrant expression. Bachmayr-Heyda and coworkers [293] have reported that the ratio of circular to linear RNA isoforms was always lower in tumor compared to normal colon samples and even lower in CRC cell lines. A negative correlation between this ratio and the proliferation index, which could infer that circRNAs accumulate in nonproliferating cells, was described. They also predicted more than 1,800 circRNAs in human normal colon mucosa and tumor samples of CRC patients, a number which is in line with other reports about circRNAs.

RNA-seq analyses revealed that circRNAs were enriched in exosomes compared to the producer cells and more than 1,000 circRNAs were identified in human serum exosomes. Exosomes contain a specific cargo of protein, mRNA, and miRNA species, which can modulate recipient cell behaviors 
and may be used as biomarkers for diagnosis of human diseases. $\mathrm{Li}$ and coworkers described for the first time the presence of abundant circRNAs in exosomes and, interestingly, revealed that serum exosomal circRNAs were able to distinguish CRC patients from healthy controls. Compared to healthy subjects, 67 circRNAs were absent and 257 new circRNA species were detected in CRC patients [294].

circRNAs have been proposed as potential biomarkers for cancer diagnosis and targeted therapy [295, 296]. Furthermore, circRNAs associate with cancer-related miRNAs acting as miRNA sponges (Figure 3), binding to RNA-associated proteins to form RNA-protein complexes, and then regulating gene transcription [297]. Memczak and coworkers [296] found that a human circRNA, antisense to the cerebellar degeneration-related protein 1 transcript (CDRlas, renamed ciRS-7: circular RNA sponge for miR-7) was densely bound by miRNA effector complexes and harbors more than 63 conserved binding sites for miR-7 and is densely bound by AGO protein but not by unrelated proteins. Thus, ciRS7 impairs the regulatory effect of miR-7 that, as we have previously described, is a tumor suppressor which is downregulated in a variety of cancers, among them CRC. As miR-7 modulates the expression of several oncogenes, ciRS7/miR-7 interaction may play important roles in cancerrelated pathways. CiRS-7 can be cleaved by miR-671 and its associated Argonaute protein, whereas it is not cleaved by miR-7 and Argonaute $2[298,299]$. The second cirRNA highly expressed in murine testes, SRY, also functions as a miR-138 sponge; the circular SRY transcript has 16 binding sites for miR-138 and coprecipitates with AGO2 [300]. Although some of the molecular and biological roles played by circRNAs have been characterized, the role of cirRNAs as new star of noncoding RNA needs to be clearly established, the biological function of some of them remains largely unknown, and very few circRNAs have been described with the potential to act as RNA sponges.

Recently, a new type of circRNAs has been described arising from transcribed exons of distinct genes affected by chromosomal translocations and that encode oncogenic fusion proteins involved in tumorigenesis. They are called fusion circRNAs and can coexist with the oncogenic fusion proteins contributing to cellular transformation, promoting cell viability and resistance upon therapy, and have tumorpromoting properties in in vivo models [301]. The presence and effects of these f-circRNAs has been shown in acute promyelocytic leukemia (f-circPR and f-circM9 arising from gene fusions $P M L / R A R A$ and $M L L / A F 9$, resp.), in Ewing sarcoma (f-circEF1 from EWSR1/FLI1 fusion), and in lung cancer (f-circEA1 from EML4/ALK1 fusion).

\section{Colorectal Cancer Treatments and Biomarkers}

6.1. Classical Therapies and Response Biomarkers in Colorectal Cancer. CRC cancer can be triggered by alteration of a plethora of molecular mechanisms, whose accumulation ultimately leads to malignant transformation. As previously mentioned, the most frequent alterations reported in the onset of CRC are as follows: oncogene activation, tumor suppressor gene inactivation, mutations in the mismatch repair (MMR) enzymes, microsatellite instability (MIN), chromosomal instability (CIN), or epigenetic alterations. Chemotherapy treatments are directed towards the reversion of some of the effects caused by these alterations, but their efficacy is highly variable and requires the development of new adjuvant therapies against different targets. In addition, the availability of new biomarkers for CRC will greatly improve the efficacy of chemotherapy.

Initial standard drugs for CRC chemotherapy (see Table 3) were 5-fluorouracil (5-FU), leucovorin, or their combination (FL); these treatments offered an overall survival of about 8 to 12 months [302]. The introduction of combined therapies with irinotecan and/or oxaliplatin (FOLFOX, FOLFIRI, CapeOX, and FOLFIRINOX) showed significant improvement in disease-free survival and overall survival [303]. A further step was achieved with the use of monoclonal antibodies targeting EGFR or the vascular endothelial growth factor receptor (VEGFR) in combination with the aforementioned chemotherapeutic agents for the treatment of metastatic CRC [304-307]. In fact, the use of these antibodies is now recommended as the standard firstline chemotherapy in this type of tumors.

The monoclonal antibodies cetuximab and panitumumab, directed against EGFR, inhibit cell proliferation and tumor growth by binding to the extracellular domain of the receptor blocking the ligand-induced phosphorylation of the cytoplasmic domain and, consequently, downstream signaling (Table 3) [308]. In this context, it is important to point out that gene mutations in proteins downstream EGFR may be responsible for cetuximab or panitumumab resistance even in combined treatments with chemotherapeutic drugs. KRAS and NRAS have been extensively studied regarding resistance to chemotherapy regimens that include these monoclonal antibodies. In fact, in patients with $R A S$ mutations in exons 2 (codons 12 and 13), 3, or 4, leading to constitutive activation of the protein, no beneficial effects are obtained with the monoclonal antibody treatments compared to standard chemotherapeutic regimens; only patients with wild-type KRAS or NRAS present a significant enhancement in survival [306, 307, 309-312]. Mutations of other molecular components of the signaling pathway downstream of EGFR (Ras-Raf-MEK-MAPKs) have also been evaluated as predictive markers for the anti-EGFR therapy. On this idea, $B R A F$ mutations seem to be an indicator of poor prognosis even in wild-type KRAS, but results are still unclear [307, 309, 313, 314].

Antiangiogenic combined therapies are also frequently used for the treatment of metastatic CRC (Table 3). The humanized monoclonal antibody bevacizumab is directed against vascular endothelial growth factor A (VEGF-A), blocking in this way its binding to VEGFR1 and VEGFR2. This growth factor is proangiogenic and has been associated with tumor vascularization, progression, and metastasis, as well as providing protection against the cytotoxic effects of chemotherapy drugs. Bevacizumab blocks neovascularization of the tumor and improves the effectiveness of antitumoral drugs [315]. Patient response to bevacizumab 
TABLE 3: Conventional chemotherapy treatments and targeted therapy for colorectal cancer.

\begin{tabular}{|c|c|}
\hline Treatment & Mechanism \\
\hline \multicolumn{2}{|r|}{ Drugs used in conventional chemotherapy treatments } \\
\hline $\begin{array}{l}\text { 5-fluorouracil } \\
(5 \mathrm{FU})\end{array}$ & $\begin{array}{l}\text { Inhibition of nucleotide biosynthesis. Prodrug. Entering the cell through uracil transport system. Intracellular } \\
\text { transformation into FdUMP, FdUTP, and FUTP. }\end{array}$ \\
\hline $\begin{array}{l}\text { Capecitabine } \\
\text { (Xeloda) }\end{array}$ & $\begin{array}{l}\text { Metabolic precursor of 5FU. Requiring the activity of carboxylesterase, cytidine deaminase, and uridine } \\
\text { phosphorylase. }\end{array}$ \\
\hline $\begin{array}{l}\text { Methotrexate } \\
\text { (MTX) }\end{array}$ & $\begin{array}{l}\text { Blocking nucleotide biosynthesis as it is a potent competitive inhibitor of dihydrofolate reductase, an enzyme that } \\
\text { participates in the tetrahydrofolate synthesis (required for de novo biosynthesis of purine and pyrimidine bases). }\end{array}$ \\
\hline $\begin{array}{l}\text { Leucovorin } \\
\text { (Wellcovorin) }\end{array}$ & $\begin{array}{l}\text { Calcium folinate. Enhancing 5FU activity. It is a 5-formyl derivative of tetrahydrofolic acid and is readily converted to } \\
\text { other reduced folic acid derivatives (e.g., tetrahydrofolate). It does not require dihydrofolate reductase activation and } \\
\text { may activate this enzyme and is used in rescue therapies after methotrexate treatment. }\end{array}$ \\
\hline $\begin{array}{l}\text { Oxaliplatin } \\
\text { (Eloxatin) }\end{array}$ & $\begin{array}{l}\text { Platinum containing compound that form inter- and intrastrand crosslinks in DNA, blocking replication and } \\
\text { transcription. } \\
\text { Approved by the FDA in } 2002 .\end{array}$ \\
\hline $\begin{array}{l}\text { Cisplatin } \\
\text { (Platinol, CDDP) }\end{array}$ & $\begin{array}{l}\text { Platinum containing compound that form inter- and intrastrand crosslinks in DNA, blocking replication and } \\
\text { transcription. }\end{array}$ \\
\hline $\begin{array}{l}\text { Irinotecan } \\
(\mathrm{CPT}-11) \\
\text { (Camptosar) }\end{array}$ & $\begin{array}{l}\text { Camptothecin derived prodrug. Irinotecan is activated by hydrolysis to SN-38, an inhibitor of topoisomerase I and, } \\
\text { thus, blocks replication and transcription. }\end{array}$ \\
\hline $\begin{array}{l}\text { Regorafenib } \\
\text { (Stivarga) }\end{array}$ & $\begin{array}{l}\text { Multikinase inhibitor which targets angiogenic, stromal, and oncogenic receptor tyrosine kinase (RTKs). } \\
\text { Approved by the FDA in } 2012 \text {. }\end{array}$ \\
\hline \multicolumn{2}{|r|}{ Combined chemotherapy regimens } \\
\hline FOLFOX & $\begin{array}{l}\text { Leucovorin (folinic acid) }+5 \mathrm{FU}+\text { oxaliplatin (stages III \& IV). } \\
\text { Approved by the FDA in } 2002 \text { for refractory tumors and in } 2004 \text { for first-line treatments of metastatic colorectal } \\
\text { cancer. }\end{array}$ \\
\hline CapOX (XELOX) & Capecitabine + oxaliplatin (stages III \& IV). \\
\hline FOLFIRI (IFL) & Leucovorin (folinic acid) $+5 \mathrm{FU}+$ irinotecan (stage IV). \\
\hline FOLFIRINOX & Leucovorin (Folinic acid) + 5FU + irinotecan + oxaliplatin (stage IV). \\
\hline FL & $\begin{array}{l}\text { 5FU + leucovorin (folinic acid). } \\
\text { Approved by the FDA in } 1991 \text { for first-line treatment of metastatic colorectal cancer. }\end{array}$ \\
\hline \multicolumn{2}{|r|}{ Monoclonal antibodies for targeted therapy of metastatic CRC } \\
\hline $\begin{array}{l}\text { Cetuximab } \\
\text { (Erbitux) }\end{array}$ & $\begin{array}{l}\text { Cetuximab is a recombinant, human-mouse chimeric IgG1 monoclonal antibody that binds specifically to the } \\
\text { extracellular domain of the human epidermal growth factor receptor (EGFR) on both normal and tumor cells and } \\
\text { competitively inhibits the binding of epidermal growth factor (EGF) and other ligands, such as transforming growth } \\
\text { factor } \alpha \text {. } \\
\text { Approved by the FDA in } 2004 \text { for use, in combination with irinotecan, for the treatment of EGFR-expressing, } \\
\text { metastatic colorectal carcinoma in patients who are refractory to irinotecan-based chemotherapy. In 2012, approved } \\
\text { for use in combination with FOLFIRI for first-line treatment of patients with wild-type K-ras. }\end{array}$ \\
\hline $\begin{array}{l}\text { Panitumumab } \\
\text { (Vectibix) }\end{array}$ & $\begin{array}{l}\text { Human } m \text { Ab against EGFR (similar to cetuximab). } \\
\text { Approved by the FDA in } 2006 \text { for the treatment of patients with EGFR-expressing, metastatic colorectal cancer with } \\
\text { disease progression. on or following fluoropyrimidine-, oxaliplatin-, and irinotecan-containing chemotherapy } \\
\text { regimens. }\end{array}$ \\
\hline $\begin{array}{l}\text { Bevacizumab } \\
\text { (Avastin) }\end{array}$ & $\begin{array}{l}\text { Bevacizumab is a recombinant humanized monoclonal antibody that binds to human vascular endothelial growth } \\
\text { factor (VEGF), thereby preventing the interaction of VEGF with its receptors on the surface of endothelial cells. } \\
\text { Approved by the FDA in } 2013 \text { for use in combination with fluoropyrimidine-irinotecan- or } \\
\text { fluoropyrimidine-oxaliplatin-based chemotherapy for the treatment of patients with metastatic colorectal cancer. }\end{array}$ \\
\hline $\begin{array}{l}\text { Ziv-aflibercept } \\
\text { (Zaltrap) }\end{array}$ & $\begin{array}{l}\text { Recombinant fusion protein consisting of VEGF-binding portions from the extracellular domains of human VEGFR1 } \\
\text { and 2, that are fused to the Fc portion of the human IgG1 immunoglobulin. It binds and inhibits VEGF-A, VEGF-B, } \\
\text { and placental growth factor. It blocks angiogenesis and decreases vascular permeability. } \\
\text { Approved by the FDA in } 2012 \text { for use in combination with FOLFIRI for the treatment of patients with metastatic } \\
\text { colorectal cancer that is resistant to or has progressed following treatment with an oxaliplatin-containing regimen. }\end{array}$ \\
\hline $\begin{array}{l}\text { Ramucirumab } \\
\text { (Cyramza) }\end{array}$ & $\begin{array}{l}\text { Human } \mathrm{mAb} \text { against VEGFR2. Inhibits angiogenesis by blocking the interaction between VEGF and VEGFR2. } \\
\text { Approved by the FDA in } 2015 \text { for use in combination with FOLFIRI for the treatment of patients with metastatic } \\
\text { colorectal cancer whose disease has progressed on a first-line regimen containing bevacizumab, oxaliplatin, and a } \\
\text { fluoropyrimidine. }\end{array}$ \\
\hline
\end{tabular}


was thought to be directly related to level of circulating VEGF-A as several studies linked high VEGF levels to a poor clinical outcome. However, the use of VEGF levels as predicted biomarker for treatment with bevacizumab is not currently advised as inconsistent data have been found. This may arise from the fact that several other molecules are involved in angiogenesis, and some of them are been analyzed as potential biomarkers for bevacizumab response [316-318].

Other approaches against the angiogenic pathway include the combined use of chemotherapic regimens with zivaflibercept or with ramucirumab. Ziv-aflibercept is a humanized fusion protein consisting of VEGF-binding portions from the extracellular domains of human VEGFR1 and VEGFR2, which are fused to the Fc portion of the human IgG1 immunoglobulin. It binds and inhibits VEGF-A, VEGF$\mathrm{B}$, and placental growth factor (PlGF), blocking in this way angiogenesis and decreasing vascular permeability. The combined treatment of metastatic CRC with FOLFIRI with zivaflibercept has shown a significant enhancement in overall survival [319]. Ramucirumab is a human monoclonal antibody against the extracellular domain of VEGFR2, interfering with VEGF-A binding and further angiogenic signaling. Its use combined with FOLFOX or FOLFIRI has also shown promising results for the treatment of patients with metastatic CRC [320-322].

The multikinase inhibitor regorafenib was approved in 2012 by the US Food and Drug Administration for the treatment of patients with metastatic CRC who have been previously treated with fluoropyrimidine-, oxaliplatin-, and irinotecan-based chemotherapy, an anti-VEGF therapy, and an anti-EGFR therapy (the latter only in those cases with wild-type KRAS). Regorafenib and its active metabolites inhibit multiple membrane-bound and intracellular kinases involved in normal cellular functions and in pathologic processes (as angiogenesis or tumor growth), including those in the RET, VEGFR1, VEGFR2, VEGFR3, KIT, PDGFR$\alpha$, PDGFR- $\beta$, FGFR1, FGFR2, TIE2, DDR2, Trk2A, Eph2A, RAF-1, BRAF, BRAFV600E, SAPK2, PTK5, and Abl pathways $[323,324]$.

Other biomarkers have been analyzed in order to check the response to monoclonal antibody treatments, mainly centered in anti-EGFR therapy. Besides KRAS and NRAS, mutations in PIK3CA, TP53, PTEN, and EGFR have been proposed as predictive biomarkers with variable success. Regarding mutations in PIK3CA (which encodes the PI3K catalytic subunit), conflicting results have been obtained [325]. It has been proposed that anti-EGFR monoclonal antibody treatments might be efficient in tumors where p53 is inactivated [326], whereas PTEN loss correlates with lack of response to cetuximab and panitumumab [325, 327].

6.2. miRNAs as Predictive Biomarkers and Therapeutic Targets in CRC. As described above, one of the major problems for the management of $\mathrm{CRC}$ is the inherent or acquired resistance to therapeutic treatments. As miRNAs are important regulators of cell signaling pathways involved in carcinogenesis, progression, invasion, angiogenesis, and metastases in CRC, they are being analyzed as potential predictive and prognostic factors, or even as therapeutic targets themselves. In fact, the involvement of the dysregulation of several miRNAs has been linked to tumor progression and response to anticancer therapies [144, 328-330]. miRNAs are also required for normal immune system development and function and aberrant expression of miRNAs has been observed in various tumor types leading to immune disorders or immune evasion. Thus, miRNAs could also be considered as potential targets in the regulation of the immune response in CRC in order to develop new therapeutic strategies [331].

miRNAs are very stable molecules that resist prolonged storage, exposure to high or low $\mathrm{pH}$ values, or even boiling and can be detected in archival tissue specimens and serum. These characteristics are quite interesting for their use as biomarkers as they can be extracted for analysis from blood, plasma, serum, and various body fluids and in frozen or formalin-fixed paraffin-embedded tissues [332, 333]. The discovery that extracellular miRNAs circulate in the bloodstream and that such circulating miRNAs are quite stable raised the possibility that miRNAs may be probed in the circulation and can also serve as novel diagnostic makers. These molecules are protected from degradation by their inclusion in lipid vesicles or by their interaction with plasma proteins, as was originally shown by El-Hefnawy and coworkers [334]. Depending on their size and mode of release from cells, miRNAs can be included into exosomes, microvesicles, or apoptotic bodies. Exosomes (50-100 nm in diameter) originate from the endosome and are released from cells when multivesicular bodies fuse with the plasma membrane; microvesicles are released from the cell through blebbing of the plasma membrane. miRNAs are also found in circulation in microparticle-free form, associated with high-density lipoproteins (HDL) or bound to RNA-binding proteins such as Ago2 or NPM1 (nucleophosmin). It is not known how these miRNA-protein complexes are released from the cell; they may be released passively, as byproducts of dead cells, or actively, in a miRNA-specific manner, through interaction with specific membrane channels or proteins [335].

Cells can select some miRNAs for cellular release while others are mainly retained within the cell. Pigati and coworkers analyzed normal and malignant epithelial cells and reported that $66 \%$ of the miRNAs were released in quantities that reflected their intracellular level and $13 \%$ were selectively retained in the cell with very low secretion, but $21 \%$ of the miRNAs were actively secreted [336]. They also observed that pre-miRNAs were also secreted together with mature miRNAs. In fact, some cells secrete preferentially the precursor forms rather than the mature ones, as reported for mesenchymal stem cells [337]. The release of miRNA-containing exosomes seems to be ceramide dependent; human CRC cells release miRNAs in ceramide-rich exosomes, and inhibition of enzymes involved in ceramide biosynthesis (as sphingomyelin phosphodiesterase 3) strongly decreases the secretion of miRNAs into exosomes [338].

6.2.1. miRNAs as Diagnostic and Prognostic Markers. miRNAs are beginning to be used as diagnostic markers of CRC [21]. Serum miR-21 and miR-92a have been analyzed as biomarkers in the diagnosis and prognosis of CRC [339]. 
Patients with advanced adenoma or with CRC showed significantly higher levels compared to healthy controls. They have been also studied in stool, but they showed lower sensitivity and specificity than in serum for the detection of CRC [340]. Elevated serum levels of miR-92a correlate well with CRC and poor survival [339], whereas miR-21 is not specific to CRC but is also found increased in the plasma of patients with other cancer types [341]. In another study, high levels of circulating miR-34a and low miR-150 levels distinguished groups of patients with polyps from those with advanced CRC, and low circulating miR-150 levels separated patients with adenomas from those with advanced cancer [342]. mir1290 has been also described as a novel biomarker for early detection, recurrence, and prognosis in human CRC [343]

In order to achieve a more precise screening for CRC, panels of plasma miRNAs have been devised: a panel consisting of eight miRNAs (miR-532-3p, miR-331, miR-195, miR-17, miR-142-3p, miR-15b, miR-532, and miR-652) could identify polyps from controls, and a panel of three miRNAs (miR-431, miR-15b, and miR-139-3p) distinguished stage IV CRC from controls [344]. miR-135b has been also found to be increased in CRC and adenomas compared to normal adjacent tissue, as well as in stool specimens where levels of this miRNA increased in patients with adenomas compared to those with CRC and were higher than in control patients or patients with inflammatory bowel disease. Moreover, miR-135b levels in stool decreased significantly after surgery, suggesting that this miRNA may act as a biomarker for early-stage CRC [345]. Fecal miR-106a has been also proposed as a useful marker for colorectal cancer patients with false-negative results in immunochemical fecal occult blood test [346].

Finally, Ahmed [347] performed a global analysis of miRNA expression in stool from 12 patients with CRC (3 per stage; 0-I, II, III, and IV) and 3 controls and found 141 miRNAs overexpressed in CRC and 61 with reduced expression. After selecting 20 miRNAs, they carried out an additional study using modified real-time quantitative PCR with stool samples from 60 patients ( 20 per group) and found 12 miRNAs overexpressed in CRC and whose expression increased with the stage (miR-7, miR-17, miR-20a, miR-21, miR-92a, miR-96, miR-106a, miR-134, miR-183, miR-196a, miR-199a-3p, and miR-214). Conversely, they also found 8 miRNAs that showed reduced expression in CRC and that decreased with stage progression (miR-9, miR-29b, miR-1275p, miR-138, miR-143, miR-146a, miR-222, and miR-938). Taking into account these finding, the authors propose that a chip can be developed to facilitate diagnosis of CRC from stool or blood samples.

In addition to diagnosis, miRNAs are beginning to be considered as promising predictive markers for response to therapy. On this idea, miR-31-3p and $-5 p$ were identified as good markers of response to cetuximab treatment in wildtype $R A S$ patients with metastatic CRC as nonresponders presented significantly higher expression levels of these two miRNAs compared to responders. However, no association between time to progression and the expression of these miRNAs was found in patients treated with panitumumab [216]. Similar associations have been found for other miRNAs and treatments. For example, miR-143 low-level expression in primary tumors was found as a good predictive factor for the effectiveness of capecitabine treatment as first-line monotherapy in patients with metastatic CRC [348]. miR320 e has been also identified in two clinical trials cohorts as a novel prognostic biomarker that is associated with adverse clinical outcome in stage III CRC patients treated with 5FU-based adjuvant chemotherapy (FOLFOX) [349]. These findings have important implications for the personalized management of CRC patients.

The presence of SNPs in specific miRNA has also been associated with prognosis and response to chemotherapy treatments in CRC. The expression of specific miR-492 variants in CRC patients has been reported in association with a better prognosis and progression-free survival [350]. Regarding response to therapy, several articles describe that KRAS-LCS6 variant is of particular interest due to an alteration in the binding site of let-7 miRNA at the $3^{\prime}$ UTR of KRas. Graziano and coworkers have reported that patients with metastatic CRC without mutations in BRAF that received anti-EGFR therapy (cetuximab plus irinotecan) showed a much lower survival if they presented the KRAS-LCS6 SNP than patients without it [351]. However, apparently opposite results were obtained by Zhang and coworkers in patients with wild-type KRAS treated only with cetuximab and no irinotecan [219]. Thus, care must be taken when considering this SNP as a predictive response parameter as it strongly depends on the chemotherapy regimen and in the mutational status of KRAS and BRAF.

6.2.2. miRNAs Involved in Chemoresistance and Radioresistance. Chemotherapy sensitivity or resistance is strongly dependent on protein targets that are epigenetically modified that involve variations in the intracellular expression of miRNAs and lncRNAs. The literature regarding the involvement of miRNAs in the acquisition of resistance in CRC cells to conventional chemotherapy agents such as 5-FU, oxaliplatin, or irinotecan is ample (Table 4). Most of the findings rely on in vitro experiments with cultured cells and some of them are even controversial. For example, cells resistant to 5-FU treatment have been reported to have increased expression of miR-192 and miR-215, whose target is thymidylate synthase (TYMS), a potential target of 5-FU. Surprisingly, however, these miRNAs seemed to increase resistance to 5-FU, rather than improving the drug's efficacy [186]. On the other hand, miR-129 or miR-203, whose mRNA target is also TYMS, behaves in a completely opposite manner; they are downregulated in 5-FU resistant cells, but their restoration induces chemosensitivity to this agent $[196,208]$. It is also somehow controversial the fact that let-7g overexpression, that belongs to a miRNA family normally described as tumor suppressors, was associated with chemoresistance to S-1 (composed of 5-FU prodrug tegafur, a dihydropyrimidine dehydrogenase inhibitor, gimestat, and an inhibitor of orotate phosphoribosyltransferase, otastat potassium, in a molar ratio of $1: 0.4: 1$ ) in colon cancer [177].

In general, those miRNAs whose expression is upregulated in chemoresistant cells repress the expression of targets related directly or indirectly to the induction of apoptosis or to the control of proliferation. An example of the former 
TABLE 4: miRNAs involved in chemoresistance in colorectal cancer treatments.

\begin{tabular}{|c|c|c|c|}
\hline miRNA & Treatment & Verified targets in CRC & References \\
\hline \multicolumn{4}{|c|}{ miRNAs overexpressed in chemoresistance to conventional drugs } \\
\hline Let-7g & S-1 & RAS, cyclin D, c-Myc, E2F, cytochrome c & [177] \\
\hline miR-10b & $5-\mathrm{FU}$ & BIM & [178] \\
\hline miR-19b & $5-\mathrm{FU}$ & SFPQ, MYBL2 & [179] \\
\hline miR-20a & 5-FU, oxaliplatin & BNIP2 & [180] \\
\hline miR-23a & $5-\mathrm{FU}$ & APAF-1 & {$[181]$} \\
\hline miR-31 & $5-\mathrm{FU}$ & $?$ & [182] \\
\hline miR-140 & $5-\mathrm{FU}$ & HDAC & [183] \\
\hline miR-148a & 5-FU, oxaliplatin & $?$ & [184] \\
\hline $\operatorname{miR}-181 b$ & S-15-FU & RAS, cyclin D, c-Myc, E2F, cytochrome c & {$[177]$} \\
\hline $\operatorname{miR} 192 / 215$ & 5-FU & TYMS, DHFR & {$[185,186]$} \\
\hline miR-203 & Oxaliplatin & ATM & {$[187]$} \\
\hline miR-224 & 5-FU & $?$ & [188] \\
\hline miR-520g & 5-FU, oxaliplatin & CDKNIA (p21) & [189] \\
\hline $\operatorname{miR}-625-3 p$ & Oxaliplatin & $?$ & [190] \\
\hline \multicolumn{4}{|c|}{ miRNAs downregulated in chemoresistance to conventional drugs } \\
\hline $\operatorname{miR}-22$ & 5-FU & BTG1 & {$[191]$} \\
\hline miR-34a & $5-\mathrm{FU}$ & SIRT1, E2F3, KIT, LDHA & {$[192-194]$} \\
\hline miR-122 & $5-\mathrm{FU}$ & PKM2 & [195] \\
\hline miR-129 & $5-\mathrm{FU}$ & BCL2, TYMS, E2F3 & [196] \\
\hline miR-133a & Oxaliplatin & RFFL & [197] \\
\hline miR-139-5p & 5-FU & NOTCH1 & [198] \\
\hline $\operatorname{miR}-141 / 200 c$ & Oxaliplatin & ZEB1 & [199] \\
\hline miR-143 & 5-FU, oxaliplatin & BCL2, NFKB, ERK5, IGF1R & {$[200,201]$} \\
\hline $\operatorname{miR} 145$ & $5-\mathrm{FU}$ & FLI-1, RAD18 & {$[202,203]$} \\
\hline miR-149 & $5-\mathrm{FU}$ & FOXM1 & [204] \\
\hline miR-153 & Oxaliplatin & FOXO3 & {$[205]$} \\
\hline miR-196a & Oxaliplatin & HOXA7, HOXB8, HOXC8, HOXD8 & [206] \\
\hline miR-200 cluster & 5-FU & EMT-related genes & {$[207]$} \\
\hline miR203 & $5-\mathrm{FU}$ & TYMS & [208] \\
\hline miR-204 & $5-\mathrm{FU}$ & HMGA2 & [209] \\
\hline miR-222 & Oxaliplatin & ADAM17 & {$[188]$} \\
\hline miR-297 & Oxaliplatin & ABCC2 & {$[210]$} \\
\hline miR-451 & Irinotecan & $\mathrm{ABCC} 1$ & [211] \\
\hline miR-497 & 5-FU, irinotecan & IGF1R & [212] \\
\hline miR-519c & 5-FU, oxaliplatin & ABCG2, ELAVL1 (HuR) & [213] \\
\hline miR-1915 & Oxaliplatin & BCL2 & {$[214]$} \\
\hline \multicolumn{4}{|c|}{ miRNAs overexpressed in chemoresistance to monoclonal antibody-based therapies } \\
\hline $\operatorname{miR}-17-3 p$ & Cetuximab & $?$ & [215] \\
\hline $\operatorname{miR}-31$ & Cetuximab & $?$ & [216] \\
\hline miR-126 & Bevacizumab, XELOX & $?$ & [217] \\
\hline $\operatorname{miR}-146-3 p$ & Cetuximab & IL1A & [215] \\
\hline miR-199a-5p/375 & Cetuximab & PHLPP1 & [218] \\
\hline miR-486-5p & Cetuximab & ARHGAP5, ST5, DOCK3, TOB1, PIK3R1 & [215] \\
\hline \multicolumn{4}{|c|}{ miRNAs downregulated in chemoresistance to monoclonal antibody-based therapies } \\
\hline Let-7 family & Cetuximab, panitumumab & KRAS & {$[215,219]$} \\
\hline miR-7 & Cetuximab & EGFR, RAF1, ERK1/2, AKT & [136] \\
\hline
\end{tabular}


is miR-153, which is upregulated in resistance to oxaliplatin in vitro and in vivo. This miRNA represses the expression of the Forkhead transcription factor FOXO3a repressing in this way apoptosis through reduced caspase-3 activation, upregulation of antiapoptotic genes, and downregulation of proapoptotic ones as PUMA and Bim [205]. Regarding dysregulation of the cell cycle, it has been proposed that miR$520 \mathrm{~g}$ may confer chemoresistance to 5 -FU and oxaliplatin in CRC cells through downregulation of p21 expression. In addition, p53 inhibits miR-520g expression and the loss of p53 function causes an increase in the expression levels of miR-520g, suggesting an important role of this miRNA as a potential target for therapy to overcome drug resistance in CRC patients [189].

miRNAs have also an impact on the resistance of CRC to targeted chemotherapy or combined regimens (Table 4). The overexpression of several miRNAs has been directly related to resistance to monoclonal antibody-based therapies. As previously discussed, miR-31 is upregulated in metastatic CRC and can be used as a predictive marker of the efficacy to cetuximab treatment but not to panitumumab. However, it is yet unclear the mechanism by which this miRNA enhances resistance [216]. Upregulation of miR-199a-5p and miR-375 induces cetuximab resistance in CRC cells by targeting the tumor suppressor PHLPP1, a negative regulator of the Akt pathway [218]. Ragusa and coworkers correlated high expression levels of miR-146b-3p and miR-486-5p with resistance to cetuximab in patients with constitutive activation of KRAS signaling, suggesting their involvement in EGFR pathway [215]. In the same study, they describe that upregulation of members of the let-7 family may enhance sensitivity of metastatic CRC to anti-EGFR treatments by targeting K-Ras mRNA. In a similar way, Suto and coworkers showed that miR-7 induced sensitivity to cetuximab in CRC cell lines by targeting EGFR and RAF1 mRNAs and suppressing ERK1/2 and phospho-Akt expression [136]. miR-126 was described as a potential tumor suppressor involved in regulation of angiogenesis and thus was suspected to be involved in the outcome of bevacizumab treatment. However, although high expression levels of miR126 in primary CRC tumors were detected in XELOX responsive patients [352], high levels of circulating miRNA-126 were associated with bevacizumab plus XELOX resistance [217].

miRNA deregulation in CRC tissues may also influence the activity of signaling pathways involved in the response to combined chemo- and radiotherapy of these tumors. Colorectal cell lines exposed to continuous low-dose radiation overexpress miR-622, and this overexpression is maintained in surviving cells. Resistance arises from a downregulation of $\mathrm{Rb}$, whose mRNA is a direct target of miR-622. In fact, overexpression of $\mathrm{Rb}$ in these cells reverses radioresistance [353]. The opposite effect has been detected for miR-630 and miR-106b, whose upregulation is directly correlated to radiosensitivity by targeting BCL2L2 and TP53RK mRNAs (miR-630), both involved in cell survival and apoptosis inhibition [354], or PTEN and p21 (miR-106b), leading to the activation of Akt which promotes cell survival and proliferation [355]. Long noncoding RNAs are also involved in radiosensitivity. The long intergenic lincRNA-p 21 has been shown to increase the sensitivity of radiotherapy for human CRC by targeting the Wnt/ $\beta$-catenin signaling pathway [287].

6.2.3. miRNA as Therapeutic Targets and Tools in CRC. As previously discussed, the expression of miRNAs is altered in CRC; some miRNAs may act as oncogenes (oncomiRs) and others as tumor suppressor genes. It is feasible to manipulate their expression by injecting miRNAs in a similar way to the use of antisense mRNAs or RNAi, blocking the activity of oncomiRs or well replacing tumor suppressor miRNAs to restore loss of function. In any case, before applying these technologies, it is essential to establish first which miRNAs are up- and downregulated in CRC, followed by loss/gain studies in vitro or in animal models. After identifying potential miRNA targets, a pharmacological analysis must be carried out with in vivo miRNA delivery studies and analysis of pharmacokinetic and pharmacodynamics. Finally, clinical trials should be carried out to evaluate efficacy and safety of the potential treatments [356].

Mature miRNAs can be inhibited using different tools: antisense oligonucleotides (anti-miRs, antagomirs, or ASOs), miRNA sponges, miRNA masking, anti-miR peptides, or small molecule inhibitors. A miRNA sponge is a transcript (mRNA) expressed from plasmids possessing multiple tandem binding sites for targeted miRNAs that block selectively a whole family of related miRNAs. For example, in breast cancer cells, Jung and coworkers demonstrated that a multipotent miRNA sponge against miR-21, miR-155, miR-221, and miR-222 strongly inhibited cell migration in a much stronger way than single miRNA targeting [357]. Sponges have been widely used to investigate miRNA function in vitro, but their utility in vivo has been limited to transgenic animals in which the sponge mRNA is overexpressed in target tissues [358]. Interestingly, it seems that some large and circular noncoding RNAs as well as pseudogene transcripts could serve as natural sponges to regulate cellular miRNA availability and lead to upregulation of downstream target genes [261, 359].

The miRNA masking technology was developed by Choi and coworkers [360] and consists of single-stranded $2^{\prime}$-Omethyl-oligonucleotides complementary to miRNA binding sites in the $3^{\prime}$ UTR of target mRNAs that disrupt the interaction of specific miRNA-mRNA pairs. An advantage of this method is that avoids potential off-target secondary effects.

Approaches using small molecule inhibitors to manipulate miRNA expression and function are also being developed. Screening of this type of compounds has identified small molecules that can specifically inhibit the expression of miRNAs, as azobenzene, which affects miR-21 expression [361], or several molecules that inhibit miR-122 that could be useful for HCV therapy [362]. These molecules do not inhibit target recognition by the miRNAs but rather modulate the transcription of targeted miRNAs. However, these molecules are still of limited therapeutic potential due to their high $\mathrm{EC}_{50}$ values and the lack of specific inhibitors for many miRNAs. On the other hand, some fluoroquinolone antibiotics, as enoxacin, have been described to enhance the effect of siRNAs or miRNAs by increasing the binding affinity of TRBP to the miRNA precursors. It does not present specificity for miRNA sequences but induces a global increased expression 


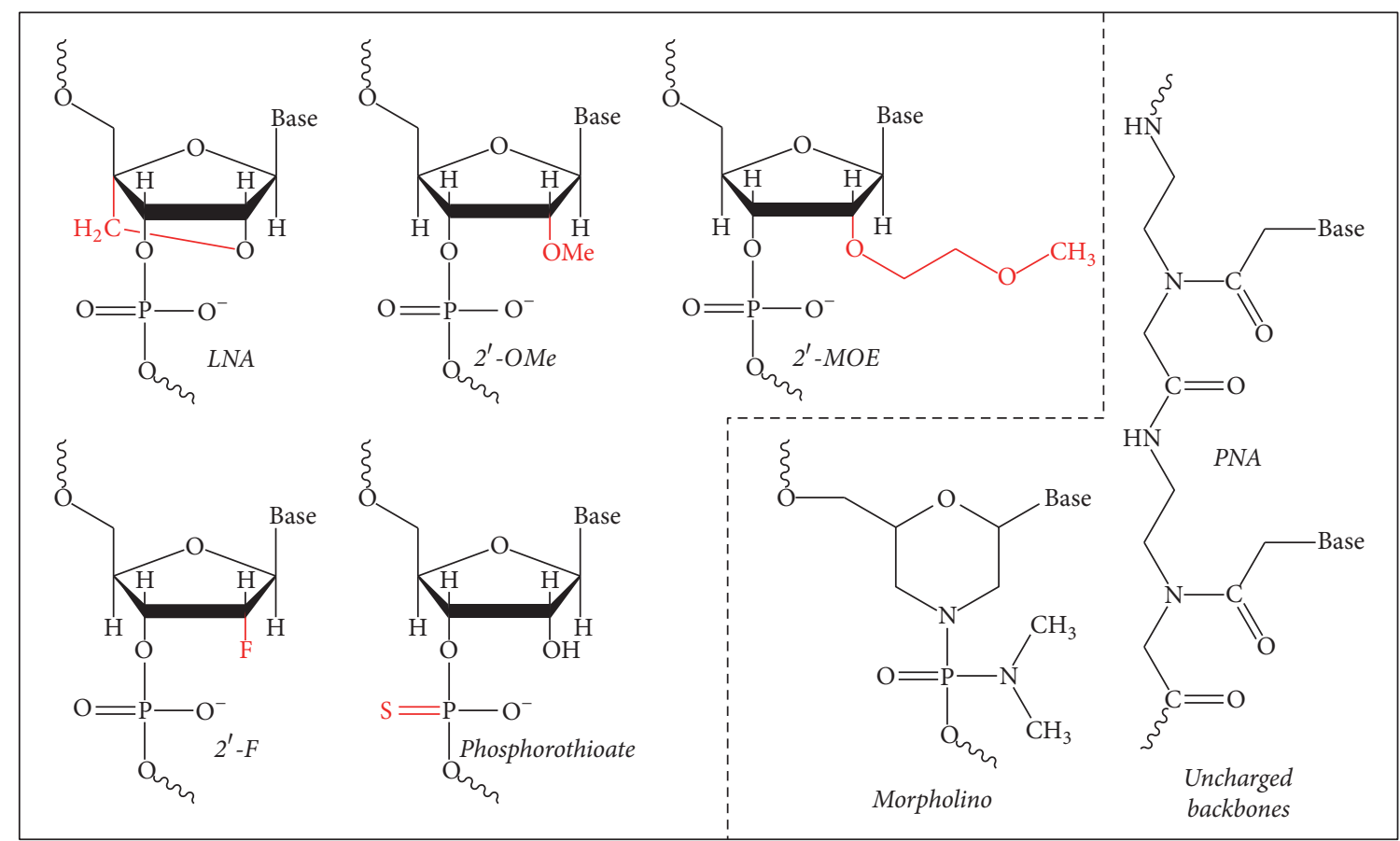

FIGURE 5: Chemical modifications of miRNA-targeting modulators. Anti-miR oligonucleotides have been chemically modified in order to achieve better stability against serum nucleases and to increase the binding affinity to targeted miRNAs. Most of the modifications are at the $2^{\prime}$ position of the sugar moiety, as $2^{\prime}-O$-methyl $\left(2^{\prime}\right.$-OMe), $2^{\prime}-O$-methoxyethyl $\left(2^{\prime}\right.$-MOE), and $2^{\prime}$-fluoro $\left(2^{\prime}-\mathrm{F}\right)$. Locked nucleic acid (LNA) is a bicyclic RNA analogue in which ribose is locked by introduction of a methylene bridge between the $2^{\prime}$ oxygen and the $4^{\prime}$ carbon of the pentose. In addition, most anti-miR oligonucleotides contain phosphorothioate backbone linkages in which sulfur replaces one of the nonbridging oxygen atoms in the phosphate group. Morpholino oligomers replace the ribose with a methylenemorpholine ring (to which bases are attached) with phosphorodiamidate linkages. PNA oligomers are oligonucleotide analogues in which the ribose-phosphate backbone has been replaced with a peptide-like structure containing $N$-(2-aminoethyl)-glycine units. Both morpholino and PNA oligomers are uncharged, which facilitates the interaction with targeted miRNAs.

of miRNAs that could be interesting for tumors where this expression is lowered [363].

Antisense inhibition of mature miRNAs is nowadays still the technology which has received more attention, mainly regarding anti-miRs or ASOs which specifically target oncomiRs. These anti-miRs bind to the miRISC complexes blocking the interaction of miRNAs with their endogenous mRNA targets. This approach has been tested in human colon carcinoma cells in culture and in mice models of CRC targeting overexpressed miRNAs. Specific silencing of miR$135 \mathrm{~b}$ has been shown to effectively inhibit tumor proliferation in mice models and to induce apoptosis in SW480 human colon carcinoma cells [364] or reduce the migratory ability in HCT-116 cells [345]. In addition to miR-135b, other potential oncogenic miRNAs have been tested using this knockdown technology as miR-20a, miR-21, miR-31, miR-95, or miR675 in other human colorectal carcinoma cell lines [124, 180, $182,276,365]$. A potential drawback of this technology is that unmodified RNA oligonucleotides are quite sensitive to serum nucleases for their potential in vivo administration. In addition, they cannot penetrate cell membranes without being modified or encapsulated to enable their entry into the cell cytoplasm. For these reasons, several lines of research are directed towards increasing resistance of antimiRs to nucleases by chemical modification, to enhance their binding affinity to mRNA targets and to improve their pharmacokinetics and pharmacodynamics in vivo (Figure 5). The $2^{\prime}$-O-methyl $\left(2^{\prime}\right.$-OMe) modification was one of the first attempts (reported in 2004) to successfully block miRISCs in Drosophila melanogaster and in human cultured cells. However, although these $2^{\prime}$-OMe modified oligonucleotides were more effective, they were still susceptible to degradation by serum nucleases. To increase resistance, oxygens in the phosphate backbone were replaced with sulfur atoms to form phosphorothioate-modified miRNAs. These modifications showed also improved pharmacokinetics [366]. Further improvement of anti-miR showed that modifications were more efficient in the $3^{\prime}$ end and that linkage of cholesterol to this end also increased the pharmacology and efficacy of specific anti-miRs [367]. Other modifications at the $2^{\prime}$ sugar position have been proposed to increase the efficacy of anti-miRs, such as $2^{\prime}$-O-methyoxyethyl $\left(2^{\prime}\right.$-MOE), $2^{\prime}$ fluoro $\left(2^{\prime}-\mathrm{F}\right)$, and locked nucleic acid (LNA; bicyclic nucleic acid with a methylene bridge between $2^{\prime}$ oxygen and the $4^{\prime}$ carbon) modifications (for review see $[329,368]$ ). Among these modifications, LNA has shown an exceptional binding affinity to miRNAs, which has made it possible to achieve efficient inhibitions with rather short sequences that bind only the seed regions of the target miRNAs [369]. 
Anti-miR peptides are artificial peptides (peptide nucleic acids or PNAs) that behave as uncharged DNA or RNA analogues in which the sugar backbone has been replaced with a peptide-like backbone which consists of $N$-(2aminoethyl)glycine units to which the nucleobases are attached. These PNAs bind to target miRNA more tightly than equivalent oligonucleotides, are more stable, and can be administered systemically with low toxicity. Similarly, morpholinos are uncharged oligonucleotide analogues with a slightly increased binding affinity to complementary miRNAs [329, 368].

Other approach for miRNA therapeutics deals with the recovery of the activity of tumor suppressor miRNAs that are downregulated or not expressed at all in CRC or other types of cancer. For this purpose, current research is using synthetic RNA duplexes that, as described above for miRNA inhibition, harbor chemical modifications to improve stability, nuclease resistance, and cellular uptake $[368,370]$. These are referred to as miRNA mimics and contain one strand identical to the miRNA of interest (guide or antisense), whereas the opposite strand (passenger or sense) is normally less stable. This passenger strand has been linked in some studies to a molecule as cholesterol or peptides to enhance cellular uptake. In addition, chemical modifications are normally introduced to avoid loading of this strand into the RISC complex, as $5^{\prime}$-O-methylation, but still allowing its degradation after separation of the antisense strand [371]. The antisense strand has to be loaded into the RISC complex and thus, its modifications for stability have to be carried out with more care to avoid interference with the formation of the complex. This therapeutic approach has been tried in colon and hepatocellular carcinomas and hepatic metastasis (that may arise from primary CRC) and, in cell culture and in mice orthotopic models, recovers the expression of downregulated tumor suppressor miRNAs miR-26a, miR33a, miR-34a, or miR-145. Overexpression of these miRNAs with mimics resulted in inhibition of cancer cell proliferation and apoptosis, or in tumor reduction, prolonged survival, and disease protection in animals [356, 372-374]. In 2013, the first miRNA mimic entered phase 1 trial in patients with primary liver cancer or metastatic cancer with liver involvement under the name of MRX34, a double-stranded RNA delivered by Smarticles (licensed liposomes which comprise different mixtures of palmitoyl oleoyl phosphatidyl choline, dioleoyloxytrimethylammoniumpropane, 1,2-dimyristoylglycerol-3hemisuccinate, and cholesterol) (http://www.mirnatherapeutics.com/) [113]. However, multiple immune-related severe adverse events were observed in patients dosed with MRX34 over the course of the trial and the company decided to halt the study.

Restoration of tumor suppressor miRNAs still presents several challenges. Treatment with the synthetic RNA duplexes may recover the expression of downregulated miRNAs, but there is always a risk of overexpressing them or that cells that normally do not express the targeted miRNAs suffer off-target effects. These potential unwanted side effects of miRNA mimics reveal the importance of improving the targeted delivery to the appropriate cells or tissues, also quite important when silencing oncomiRs.
As discussed above, the use of miRNA therapeutics in vivo presents considerably more challenges than research in vitro. Much progress has been made regarding the resistance of therapeutic miRNAs to nucleases or to increase the binding affinity of anti-miRs or miRNA mimics to mRNA targets in the miRISC complexes. However, the main challenge for development of miRNA-based therapeutics is the design of efficient and safe methods for the delivery in vivo of miRNAs to targeted tissues or cells $[329,368]$. Research has been mainly centered in delivery of siRNAs, but these advances can be readily applied to miRNA mimics and anti-miRs.

Conjugation of cholesterol to the $3^{\prime}$ end of miRNAs was one of the first attempts; it allowed incorporation of siRNAs or miRNA mimics into HDL and LDL lipoproteins [375]. Since them, several other molecules have been checked, as $\alpha$-tocopherol linked to the $5^{\prime}$ end of the antisense strand, receptor-targeting ligands for cell binding (i.e., CpGcontaining oligonucleotides directed for cells expressing TLR9 receptor), or cell-penetrating peptides for crossing cellsurface or endosomal membranes, as pHLIP. This peptide is a 38-amino-acid-long hydrophobic, negatively charged peptide that, under slightly acidic conditions (around pH 6.5), inserts as a helix into cell membranes. This peptide was unsuccessful when used with oligonucleotides but worked efficiently with PNAs linked via a disulfide bond [376].

Lentivirus- or adeno-associated virus-based miRNA expression constructs have been also studied for the delivery of tumor suppressor miRNAs, and some of these constructs are being used in several clinical trials for gene therapy. Liposome-based methods have been also widely tested for the encapsulation of siRNAs and miRNAs or analogues. These liposomes can even be coated with carbohydrates, peptides, or antibodies to direct them towards cell types that express specific receptors or antigens. Similarly, siRNAs or miRNAs can be incorporated in polymer-based nanoparticles $(10-100 \mathrm{~nm})$, as those containing polyethyleneimine that have been reported to be efficient for the therapy of CRC in cell culture and mouse xenografts by restoring miR-33a or miR145 function [373, 374]. Antibody-based methods have been also described. The most common approach is to design chimeric proteins consisting of an RNA-binding protein (as protamine or synthetic positively charged peptides) fused to Fab or single-chain variable fragments from antibodies directed towards antigens expressed in targeted cells.

Several challenges remain to be addressed before miRNA therapeutics can reach safely the clinic. A first one is associated with the fact that, from the 2588 mature miRNAs described so far in humans (miRBase 21), only around 200 are sufficiently expressed to be feasible targets for therapy [329]. Many of these miRNAs belong to families with similar seed regions and thus, it is quite normal that, for example, an anti$\mathrm{miR}$ directed against one member of one of these families may affect also other miRNAs with similar or identical seed region under physiological conditions. A second important aspect to be considered is that miRNA mimics or antimiRs can elicit an immunological response mainly through the innate immune system, whose cells express Toll-like receptors (TLRs) [377]. These receptors recognize dsRNAs (TLR3) or single-stranded GU-rich RNAs (TLR7 and TLR8) 
and evoke an interferon- $\alpha$ response by plasmacytoid dendritic cells [378]. This response can be reduced by chemical modification of the sense strand; on this idea, Hornung and coworkers observed that LNA modifications strongly reduced the immunostimulatory effects of siRNAs [378]. Although these modifications may be efficient in order to get nuclease resistance or stability of miRNAs, some of them may elicit other undesirable responses, affecting, for example, blood coagulation, activation of the complement cascade, the immune system (phosphorothioate-containing oligonucleotides), or liver toxicity (i.e., LNA-modified antimiRs) [329]. Finally, much care has to be taken when administering anti-miRs in vivo as blocking the function of certain oncomiRs may negatively affect physiological responses where those miRs exert specific functions. Thus, cell or tissue targeting is essential to avoid these potential problems. In addition, it is also difficult to ensure whether the appropriate dose of miRNA reaches targeted cells and becomes incorporated into RISCs. Some of these potential problems could be addressed with more efficient delivery systems in order to direct miRNAs towards specific cells or tissues, facilitate intracellular transport, and further release into the cytoplasm. This could allow decreasing the therapeutic dose of miRNA mimic or anti-miR in vivo minimizing adverse effects.

\section{Conflicts of Interest}

The authors declare that they have no conflicts of interest.

\section{Acknowledgments}

This work was supported by Ministerio de Ciencia e Innovación (MICINN) with Grant BFU2008-04758 and by Santander-UCM with Grant PR26/16-20323.

\section{References}

[1] M. J. Arends, "Pathways of colorectal carcinogenesis," Applied Immunohistochemistry and Molecular Morphology, vol. 21, no. 2, pp. 97-102, 2013.

[2] J. I. Barrasa, N. Olmo, M. A. Lizarbe, and J. Turnay, "Bile acids in the colon, from healthy to cytotoxic molecules," Toxicology in Vitro, vol. 27, no. 2, pp. 964-977, 2013.

[3] K. Tariq and K. Ghias, "Colorectal cancer carcinogenesis: a review of mechanisms," Cancer Biology and Medicine, vol. 13, no. 1, pp. 120-135, 2016.

[4] S. Tejpar and E. Van Cutsem, "Molecular and genetic defects in colorectal tumorigenesis," Bailliere's Best Practice and Research in Clinical Gastroenterology, vol. 16, no. 2, pp. 171-185, 2002.

[5] D. L. Worthley, V. L. Whitehall, K. J. Spring, and B. A. Leggett, "Colorectal carcinogenesis: road maps to cancer," World Journal of Gastroenterology, vol. 13, no. 28, pp. 3784-3791, 2007.

[6] E. Lages, H. Ipas, A. Guttin, H. Nesr, F. Berger, and J.-P. Issartel, "MicroRNAs: molecular features and role in cancer," Frontiers in Bioscience, vol. 17, no. 7, pp. 2508-2540, 2011.

[7] G. A. Calin, C. Sevignani, C. D. Dumitru et al., "Human microRNA genes are frequently located at fragile sites and genomic regions involved in cancers," Proceedings of the
National Academy of Sciences of the United States of America, vol. 101, no. 9, pp. 2999-3004, 2004.

[8] C. M. Croce, "Causes and consequences of microRNA dysregulation in cancer," Nature Reviews Genetics, vol. 10, no. 10, pp. 704-714, 2009.

[9] M. Esteller, "Non-coding RNAs in human disease," Nature Reviews Genetics, vol. 12, no. 12, pp. 861-874, 2011.

[10] M. Ha and V. N. Kim, "Regulation of microRNA biogenesis," Nature Reviews Molecular Cell Biology, vol. 15, no. 8, pp. 509524, 2014.

[11] A. Hata and R. Kashima, "Dysregulation of microRNA biogenesis machinery in cancer," Critical Reviews in Biochemistry and Molecular Biology, vol. 51, no. 3, pp. 121-134, 2016.

[12] J. Krol, I. Loedige, and W. Filipowicz, "The widespread regulation of microRNA biogenesis, function and decay," Nature Reviews Genetics, vol. 11, no. 9, pp. 597-610, 2010.

[13] D. A. Hill, J. Ivanovich, J. R. Priest et al., "DICER1 mutations in familial pleuropulmonary blastoma," Science, vol. 325, no. 5943, p. 965, 2009.

[14] S. A. Melo, S. Ropero, C. Moutinho et al., "A TARBP2 mutation in human cancer impairs microRNA processing and DICER1 function," Nature Genetics, vol. 41, no. 3, pp. 365-370, 2009.

[15] A. E. Pasquinelli, "MicroRNAs and their targets: recognition, regulation and an emerging reciprocal relationship," Nature Reviews Genetics, vol. 13, no. 4, pp. 271-282, 2012.

[16] F. Radtke and H. Clevers, "Self-renewal and cancer of the gut: two sides of a coin," Science, vol. 307, no. 5717, pp. 1904-1909, 2005.

[17] N. Barker, M. V. De Wetering, and H. Clevers, "The intestinal stem cell," Genes and Development, vol. 22, no. 14, pp. 1856-1864, 2008 .

[18] R. G. J. Vries, M. Huch, and H. Clevers, "Stem cells and cancer of the stomach and intestine," Molecular Oncology, vol. 4, no. 5, pp. 373-384, 2010.

[19] L. A. Torre, R. L. Siegel, E. M. Ward, and A. Jemal, "Global cancer incidence and mortality rates and trends-an update," Cancer Epidemiology Biomarkers and Prevention, vol. 25, no. 1, pp. 16-27, 2016.

[20] E. R. Fearon and B. Vogelstein, "A genetic model for colorectal tumorigenesis," Cell, vol. 61, no. 5, pp. 759-767, 1990.

[21] A. J. Yiu and C. Y. Yiu, "Biomarkers in colorectal cancer," Anticancer Research, vol. 36, no. 3, pp. 1093-1102, 2016.

[22] R. C. Bates and A. M. Mercurio, "The epithelial-mesenchymal transition (EMT) and colorectal cancer progression," Cancer Biology and Therapy, vol. 4, no. 4, pp. 365-370, 2005.

[23] V. J. Findlay, C. Wang, D. K. Watson, and E. R. Camp, "Epithelial-to-mesenchymal transition and the cancer stem cell phenotype: insights from cancer biology with therapeutic implications for colorectal cancer," Cancer Gene Therapy, vol. 21, no. 5, pp. 181-187, 2014.

[24] M. A. Puglisi, V. Tesori, W. Lattanzi, G. B. Gasbarrini, and A. Gasbarrini, "Colon cancer stem cells: controversies and perspectives," World Journal of Gastroenterology, vol. 19, no. 20, pp. 2997-3006, 2013.

[25] B. M. Boman and M. S. Wicha, "Cancer stem cells: a step toward the cure," Journal of Clinical Oncology, vol. 26, no. 17, pp. 27952799, 2008.

[26] J. A. Martínez-Climent, E. J. Andreu, and F. Prosper, "Somatic stem cells and the origin of cancer," Clinical and Translational Oncology, vol. 8, no. 9, pp. 647-663, 2006. 
[27] M. Baiocchi, M. Biffoni, L. Ricci-Vitiani, E. Pilozzi, and R. De Maria, "New models for cancer research: human cancer stem cell xenografts," Current Opinion in Pharmacology, vol. 10, no. 4, pp. 380-384, 2010.

[28] C. A. O’Brien, A. Pollett, S. Gallinger, and J. E. Dick, "A human colon cancer cell capable of initiating tumour growth in immunodeficient mice," Nature, vol. 445, no. 7123, pp. 106-110, 2007.

[29] L. Ricci-Vitiani, D. G. Lombardi, E. Pilozzi et al., "Identification and expansion of human colon-cancer-initiating cells," Nature, vol. 445, no. 7123, pp. 111-115, 2007.

[30] J. Neuzil, M. Stantic, R. Zobalova et al., "Tumour-initiating cells vs. cancer 'stem' cells and CD133: what's in the name?" Biochemical and Biophysical Research Communications, vol. 355, no. 4, pp. 855-859, 2007.

[31] P. Dalerba, S. J. Dylla, I.-K. Park et al., "Phenotypic characterization of human colorectal cancer stem cells," Proceedings of the National Academy of Sciences of the United States of America, vol. 104, no. 24, pp. 10158-10163, 2007.

[32] A. P. Feinberg and B. Vogelstein, "Hypomethylation distinguishes genes of some human cancers from their normal counterparts," Nature, vol. 301, no. 5895, pp. 89-92, 1983.

[33] F. J. Carmona and M. Esteller, "Epigenomics of human colon cancer," Mutation Research-Fundamental and Molecular Mechanisms of Mutagenesis, vol. 693, no. 1-2, pp. 53-60, 2010.

[34] A. Goel and C. R. Boland, "Epigenetics of colorectal cancer," Gastroenterology, vol. 143, no. 6, pp. 1442-1460.el, 2012.

[35] L. Song and Y. Li, “The Role of Stem Cell DNA Methylation in Colorectal Carcinogenesis," Stem Cell Reviews and Reports, vol. 12, no. 5, pp. 573-583, 2016.

[36] J. I. Barrasa, N. Olmo, A. Santiago-Gómez et al., "Histone deacetylase inhibitors upregulate MMP11 gene expression through Sp1/Smad complexes in human colon adenocarcinoma cells," Biochimica et Biophysica Acta, vol.1823, no. 2, pp. 570-581, 2012.

[37] J. Y. Fang, Y. X. Chen, J. Lu et al., "Epigenetic modification regulates both expression of tumor-associated genes and cell cycle progressing in human colon cancer cell lines: colo-320 and SW1116," Cell Research, vol. 14, no. 3, pp. 217-226, 2004.

[38] A. Guzmán-Aránguez, N. Olmo, J. Turnay et al., "Differentiation of human colon adenocarcinoma cells alters the expression and intracellular localization of annexins A1, A2, and A5," Journal of Cellular Biochemistry, vol. 94, no. 1, pp. 178-193, 2005.

[39] E. Lecona, J. I. Barrasa, N. Olmo, B. Llorente, J. Turnay, and M. A. Lizarbe, "Upregulation of annexin A1 expression by butyrate in human colon adenocarcinoma cells: role of p53, NF-Y, and p38 mitogen-activated protein kinase," Molecular and Cellular Biology, vol. 28, no. 15, pp. 4665-4674, 2008.

[40] J. I. Barrasa, A. Santiago-Gómez, N. Olmo, M. A. Lizarbe, and J. Turnay, "Resistance to butyrate impairs bile acid-induced apoptosis in human colon adenocarcinoma cells via up-regulation of Bcl-2 and inactivation of Bax," Biochimica et Biophysica Acta (BBA)_Molecular Cell Research, vol.1823, no. 12, pp. 2201-2209, 2012.

[41] I. L. De Silanes, N. Olmo, J. Turnay et al., "Acquisition of resistance to butyrate enhances survival after stress and induces malignancy of human colon carcinoma cells," Cancer Research, vol. 64, no. 13, pp. 4593-4600, 2004.

[42] N. Olmo, J. Turnay, E. Lecona et al., "Acquisition of resistance to butyrate induces resistance to luminal components and other types of stress in human colon adenocarcinoma cells," Toxicology in Vitro, vol. 21, no. 2, pp. 254-261, 2007.
[43] N. Olmo, J. Turnay, P. Pérez-Ramos et al., "In vitro models for the study of the effect of butyrate on human colon adenocarcinoma cells," Toxicology in Vitro, vol. 21, no. 2, pp. 262-270, 2007.

[44] P. Pérez-Ramos, N. Olmo, J. Turnay et al., "Effect of bile acids on butyrate-sensitive and -resistant human colon adenocarcinoma cells," Nutrition and Cancer, vol. 53, no. 2, pp. 208-219, 2005.

[45] S. Shukla and S. M. Meeran, "Epigenetics of cancer stem cells: pathways and therapeutics," Biochimica et Biophysica Acta, vol. 1840, no. 12, pp. 3494-3502, 2014.

[46] M. Chen, J. Chen, and D. Zhang, "Exploring the secrets of long noncoding RNAs," International Journal of Molecular Sciences, vol. 16, no. 3, pp. 5467-5496, 2015.

[47] J. Wang, Y.-X. Song, B. Ma et al., "Regulatory roles of noncoding RNAs in colorectal cancer," International Journal of Molecular Sciences, vol. 16, no. 8, pp. 19886-19919, 2015.

[48] D. Fanale, M. Castiglia, V. Bazan, and A. Russo, "Involvement of non-coding RNAs in chemo- and radioresistance of colorectal cancer," Advances in Experimental Medicine and Biology, vol. 937, pp. 207-228, 2016.

[49] M. Matsui and D. R. Corey, "Non-coding RNAs as drug targets," Nature Reviews Drug Discovery, 2016.

[50] S. M. Berget, C. Moore, and P. A. Sharp, "Spliced segments at the $5^{\prime}$ terminus of adenovirus 2 late mRNA," Proceedings of the National Academy of Sciences of the United States of America, vol. 74, no. 8, pp. 3171-3175, 1977.

[51] J. Liu and E. S. Maxwell, "Mouse U14 snRNA is encoded in an intron of the mouse cognate hsc70 heat shock gene," Nucleic Acids Research, vol. 18, no. 22, pp. 6565-6571, 1990.

[52] E. S. Maxwell and M. J. Fournier, “The small nucleolar RNAs," Annual Review of Biochemistry, vol. 64, pp. 897-934, 1995.

[53] J. E. Wilusz, H. Sunwoo, and D. L. Spector, "Long noncoding RNAs: functional surprises from the RNA world," Genes and Development, vol. 23, no. 13, pp. 1494-1504, 2009.

[54] A. Fire, S. Xu, M. K. Montgomery, S. A. Kostas, S. E. Driver, and C. C. Mello, "Potent and specific genetic interference by doublestranded RNA in caenorhabditis elegans," Nature, vol. 391, no. 6669, pp. 806-811, 1998.

[55] J. K. W. Lam, M. Y. T. Chow, Y. Zhang, and S. W. S. Leung, "siRNA versus miRNA as therapeutics for gene silencing," Molecular Therapy-Nucleic Acids, vol. 4, no. 9, article no. e252, 2015.

[56] D. P. Bartel, "MicroRNAs: genomics, biogenesis, mechanism, and function," Cell, vol. 116, no. 2, pp. 281-297, 2004.

[57] V. N. Kim, "Small RNAs: classification, biogenesis, and function," Molecules and Cells, vol. 19, no. 1, pp. 1-15, 2005.

[58] G. A. Calin, C. D. Dumitru, M. Shimizu et al., "Frequent deletions and down-regulation of micro-RNA genes miR15 and miR16 at 13q14 in chronic lymphocytic leukemia," Proceedings of the National Academy of Sciences of the United States of America, vol. 99, no. 24, pp. 15524-15529, 2002.

[59] X. Chen, S. Fan, and E. Song, "Noncoding RNAs: new players in cancers," in The Long and Short Non-coding RNAs in Cancer Biology, vol. 927 of Advances in Experimental Medicine and Biology, pp. 1-47, Springer, Singapore, 2016.

[60] A. Kozomara and S. Griffiths-Jones, "MiRBase: annotating high confidence microRNAs using deep sequencing data," Nucleic Acids Research, vol. 42, no. 1, pp. D68-D73, 2014.

[61] V. Ambros, B. Bartel, D. P. Bartel et al., "A uniform system for microRNA annotation," RNA, vol. 9, no. 3, pp. 277-279, 2003.

[62] S. Griffiths-Jones, “The microRNA registry," Nucleic Acids Research, vol. 32, pp. D109-D111, 2004. 
[63] Z. Du, T. Sun, E. Hacisuleyman et al., "Integrative analyses reveal a long noncoding RNA-mediated sponge regulatory network in prostate cancer," Nature Communications, vol. 7, Article ID 10982, 2016.

[64] N. Barron, N. Sanchez, P. Kelly, and M. Clynes, "MicroRNAs: tiny targets for engineering $\mathrm{CHO}$ cell phenotypes?" Biotechnology Letters, vol. 33, no. 1, pp. 11-21, 2011.

[65] E. F. Finnegan and A. E. Pasquinelli, "MicroRNA biogenesis: regulating the regulators," Critical Reviews in Biochemistry and Molecular Biology, vol. 48, no. 1, pp. 51-68, 2013.

[66] R. B. Donker, J. F. Mouillet, T. Chu et al., "The expression profile of C19MC microRNAs in primary human trophoblast cells and exosomes," Molecular Human Reproduction, vol. 18, no. 8, pp. 417-424, 2012.

[67] X. C. Ding, J. Weiler, and H. Großhans, "Regulating the regulators: mechanisms controlling the maturation of microRNAs," Trends in Biotechnology, vol. 27, no. 1, pp. 27-36, 2009.

[68] M. R. Fabian and N. Sonenberg, "The mechanics of miRNAmediated gene silencing: a look under the hood of miRISC," Nature Structural and Molecular Biology, vol. 19, no. 6, pp. 586593, 2012.

[69] A. Gurtner, E. Falcone, F. Garibaldi, and G. Piaggio, "Dysregulation of microRNA biogenesis in cancer: the impact of mutant p53 on Drosha complex activity," Journal of Experimental and Clinical Cancer Research, vol. 35, no. 1, article no. 45, 2016.

[70] J. Lu, G. Getz, E. A. Miska et al., "MicroRNA expression profiles classify human cancers," Nature, vol. 435, no. 7043, pp. 834-838, 2005.

[71] R.-J. Lin, Y.-C. Lin, J. Chen et al., "microRNA signature and expression of Dicer and Drosha can predict prognosis and delineate risk groups in neuroblastoma," Cancer Research, vol. 70, no. 20, pp. 7841-7850, 2010.

[72] G. Martello, A. Rosato, F. Ferrari et al., "A microRNA targeting dicer for metastasis control," Cell, vol. 141, no. 7, pp. 1195-1207, 2010.

[73] W. M. Merritt, Y. G. Lin, L. Y. Han et al., "Dicer, Drosha, and outcomes in patients with ovarian cancer," New England Journal of Medicine, vol. 359, no. 25, pp. 2641-2650, 2008.

[74] S. Lin and R. I. Gregory, "MicroRNA biogenesis pathways in cancer," Nature Reviews Cancer, vol. 15, no. 6, pp. 321-333, 2015.

[75] S. A. Melo, C. Moutinho, S. Ropero et al., "A genetic defect in exportin-5 traps precursor MicroRNAs in the nucleus of cancer cells," Cancer Cell, vol. 18, no. 4, pp. 303-315, 2010.

[76] B. Kim, J.-H. Lee, J. W. Park et al., "An essential microRNA maturing microprocessor complex component DGCR8 is upregulated in colorectal carcinomas," Clinical and Experimental Medicine, vol. 14, no. 3, pp. 331-336, 2014.

[77] C. Faber, D. Horst, F. Hlubek, and T. Kirchner, “Overexpression of Dicer predicts poor survival in colorectal cancer," European Journal of Cancer, vol. 47, no. 9, pp. 1414-1419, 2011.

[78] J. Stratmann, C.-J. Wang, S. Gnosa et al., "Dicer and miRNA in relation to clinicopathological variables in colorectal cancer patients," BMC Cancer, vol. 11, article no. 345, 2011.

[79] D. J. Papachristou, A. Korpetinou, E. Giannopoulou et al., "Expression of the ribonucleases Drosha, Dicer, and Ago2 in colorectal carcinomas," Virchows Archiv, vol. 459, no. 4, pp. 431440, 2011.

[80] R. C. Lee, R. L. Feinbaum, and V. Ambros, "The C. elegans heterochronic gene lin- 4 encodes small RNAs with antisense complementarity to lin-14," Cell, vol. 75, no. 5, pp. 843-854, 1993.
[81] B. Wightman, I. Ha, and G. Ruvkun, "Posttranscriptional regulation of the heterochronic gene lin-14 by lin- 4 mediates temporal pattern formation in C. elegans," Cell, vol. 75, no. 5, pp. 855-862, 1993.

[82] A. E. Pasquinelli, B. J. Reinhart, F. Slack et al., "Conservation of the sequence and temporal expression of let-7 heterochronic regulatory RNA," Nature, vol. 408, no. 6808, pp. 86-89, 2000.

[83] B. J. Reinhart, F. J. Slack, M. Basson et al., "The 21-nucleotide let-7 RNA regulates developmental timing in Caenorhabditis elegans," Nature, vol. 403, no. 6772, pp. 901-906, 2000.

[84] M. Lagos-Quintana, R. Rauhut, W. Lendeckel, and T. Tuschl, "Identification of novel genes coding for small expressed RNAs," Science, vol. 294, no. 5543, pp. 853-858, 2001.

[85] N. C. Lau, L. P. Lim, E. G. Weinstein, and D. P. Bartel, "An abundant class of tiny RNAs with probable regulatory roles in Caenorhabditis elegans," Science, vol. 294, no. 5543, pp. 858-862, 2001.

[86] R. C. Lee and V. Ambros, "An extensive class of small RNAs in Caenorhabditis elegans," Science, vol. 294, no. 5543, pp. 862-864, 2001.

[87] M. Z. Michael, S. M. O’Connor, N. G. Van Holst Pellekaan, G. P. Young, and R. J. James, "Reduced accumulation of specific MicroRNAs in colorectal neoplasia," Molecular Cancer Research, vol. 1, no. 12, pp. 882-891, 2003.

[88] J. Takamizawa, H. Konishi, K. Yanagisawa et al., "Reduced expression of the let-7 microRNAs in human lung cancers in association with shortened postoperative survival," Cancer Research, vol. 64, no. 11, pp. 3753-3756, 2004.

[89] A. Cimmino, G. A. Calin, M. Fabbri et al., "miR-15 and miR-16 induce apoptosis by targeting BCL2," Proceedings of the National Academy of Sciences of the United States of America, vol. 102, no. 39, pp. 13944-13949, 2005.

[90] M. V. Iorio, M. Ferracin, C.-G. Liu et al., "MicroRNA gene expression deregulation in human breast cancer," Cancer Research, vol. 65, no. 16, pp. 7065-7070, 2005.

[91] S. M. Johnson, H. Grosshans, J. Shingara et al., "RAS is regulated by the let-7 microRNA family," Cell, vol. 120, no. 5, pp. 635-647, 2005.

[92] K. A. O’Donnell, E. A. Wentzel, K. I. Zeller, C. V. Dang, and J. T. Mendell, "c-Myc-regulated microRNAs modulate E2F1 expression," Nature, vol. 435, no. 7043, pp. 839-843, 2005.

[93] Y. Akao, Y. Nakagawa, and T. Naoe, "MicroRNAs 143 and 145 are possible common onco-microRNAs in human cancers," Oncology Reports, vol. 16, no. 4, pp. 845-850, 2006.

[94] G. T. Bommer, I. Gerin, Y. Feng et al., "p53-mediated activation of miRNA34 candidate tumor-suppressor genes," Current Biology, vol. 17, no. 15, pp. 1298-1307, 2007.

[95] T.-C. Chang, E. A. Wentzel, O. A. Kent et al., “Transactivation of miR-34a by p53 broadly influences gene expression and promotes apoptosis," Molecular Cell, vol. 26, no. 5, pp. 745-752, 2007.

[96] L. He, X. He, L. P. Lim et al., "A microRNA component of the p53 tumour suppressor network," Nature, vol. 447, no. 7148, pp. 1130-1134, 2007.

[97] L. He, X. He, S. W. Lowe, and G. J. Hannon, "microRNAs join the p53 network-another piece in the tumour-suppression puzzle," Nature Reviews Cancer, vol. 7, no. 11, pp. 819-822, 2007.

[98] L. He, J. M. Thomson, M. T. Hemann et al., "A microRNA polycistron as a potential human oncogene," Nature, vol. 435, no. 7043, pp. 828-833, 2005. 
[99] N. Raver-Shapira, E. Marciano, E. Meiri et al., "Transcriptional activation of miR-34a contributes to p53-mediated apoptosis," Molecular Cell, vol. 26, no. 5, pp. 731-743, 2007.

[100] W. Tam and J. E. Dahlberg, "miR-155/BIC as an oncogenic microRNA," Genes Chromosomes and Cancer, vol. 45, no. 2, pp. 211-212, 2006.

[101] V. Tarasov, P. Jung, B. Verdoodt et al., "Differential regulation of microRNAs by p53 revealed by massively parallel sequencing: miR-34a is a p53 target that induces apoptosis and G1-arrest," Cell Cycle, vol. 6, no. 13, pp. 1586-1593, 2007.

[102] P. M. Voorhoeve, C. le Sage, M. Schrier et al., "A genetic screen implicates miRNA-372 and miRNA-373 as oncogenes in testicular germ cell tumors," Cell, vol. 124, no. 6, pp. 1169-1181, 2006.

[103] M. S. Ebert, J. R. Neilson, and P. A. Sharp, "MicroRNA sponges: competitive inhibitors of small RNAs in mammalian cells," Nature Methods, vol. 4, no. 9, pp. 721-726, 2007.

[104] J. M. Franco-Zorrilla, A. Valli, M. Todesco et al., "Target mimicry provides a new mechanism for regulation of microRNA activity," Nature Genetics, vol. 39, no. 8, pp. 10331037, 2007.

[105] Q. Huang, K. Gumireddy, M. Schrier et al., “The microRNAs miR-373 and miR-520c promote tumour invasion and metastasis," Nature Cell Biology, vol. 10, no. 2, pp. 202-210, 2008.

[106] L. Ma, J. Teruya-Feldstein, and R. A. Weinberg, "Tumour invasion and metastasis initiated by microRNA-10b in breast cancer," Nature, vol. 449, no. 7163, pp. 682-688, 2007.

[107] M. S. Nicoloso, R. Spizzo, M. Shimizu, S. Rossi, and G. A. Calin, "MicroRNAs-the micro steering wheel of tumour metastases," Nature Reviews Cancer, vol. 9, no. 4, pp. 293-302, 2009.

[108] S. F. Tavazoie, C. Alarcón, T. Oskarsson et al., "Endogenous human microRNAs that suppress breast cancer metastasis," Nature, vol. 451, no. 7175, pp. 147-152, 2008.

[109] X. Chen, Y. Ba, L. Ma et al., "Characterization of microRNAs in serum: a novel class of biomarkers for diagnosis of cancer and other diseases," Cell Research, vol. 18, no. 10, pp. 997-1006, 2008.

[110] S. S. C. Chim, T. K. F. Shing, E. C. W. Hung et al., "Detection and characterization of placental microRNAs in maternal plasma," Clinical Chemistry, vol. 54, no. 3, pp. 482-490, 2008.

[111] C. H. Lawrie, S. Gal, H. M. Dunlop et al., "Detection of elevated levels of tumour-associated microRNAs in serum of patients with diffuse large B-cell lymphoma," British Journal of Haematology, vol. 141, no. 5, pp. 672-675, 2008.

[112] P. S. Mitchell, R. K. Parkin, E. M. Kroh et al., "Circulating microRNAs as stable blood-based markers for cancer detection," Proceedings of the National Academy of Sciences of the United States of America, vol. 105, no. 30, pp. 10513-10518, 2008.

[113] A. Bouchie, "First microRNA mimic enters clinic," Nature Biotechnology, vol. 31, no. 7, p. 577, 2013.

[114] C.-W. Wu, Y.-J. Dong, Q.-Y. Liang et al., "MicroRNA-18a attenuates DNA damage repair through suppressing the expression of ataxia telangiectasia mutated in colorectal cancer," PLoS ONE, vol. 8, no. 2, Article ID e57036, 2013.

[115] I. A. Asangani, S. A. K. Rasheed, D. A. Nikolova et al., "MicroRNA-21 (miR-21) post-transcriptionally downregulates tumor suppressor Pdcd4 and stimulates invasion, intravasation and metastasis in colorectal cancer," Oncogene, vol. 27, no. 15, pp. 2128-2136, 2008.

[116] Y. Yang, J.-J. Yang, H. Tao, and W.-S. Jin, "MicroRNA-21 controls hTERT via PTEN in human colorectal cancer cell proliferation," Journal of Physiology and Biochemistry, vol. 71, no. 1, pp. 59-68, 2015.
[117] Y. Yu, S. S. Kanwar, B. B. Patel et al., "MicroRNA-21 induces stemness by downregulating transforming growth factor beta receptor 2 (TGF $\beta \mathrm{r} 2$ ) in colon cancer cells," Carcinogenesis, vol. 33, no. 1, pp. 68-76, 2012.

[118] W. Tang, Y. Zhu, J. Gao et al., "MicroRNA-29a promotes colorectal cancer metastasis by regulating matrix metalloproteinase 2 and E-cadherin via KLF4," British Journal of Cancer, vol. 110, no. 2, pp. 450-458, 2014.

[119] T. Chen, L.-Q. Yao, Q. Shi et al., "MicroRNA-31 contributes to colorectal cancer development by targeting factor inhibiting HIF-1 $\alpha$ (FIH-1)," Cancer Biology and Therapy, vol. 15, no. 5, pp. 516-523, 2014.

[120] D. Sun, F. Yu, Y. Ma et al., "MicroRNA-31 activates the RAS pathway and functions as an oncogenic MicroRNA in human colorectal cancer by repressing RAS p21 GTPase activating protein 1 (RASA1)," The Journal of Biological Chemistry, vol. 288, no. 13, pp. 9508-9518, 2013.

[121] R.-S. Xu, X.-D. Wu, S.-Q. Zhang et al., "The tumor suppressor gene RhoBTB1 is a novel target of miR-31 in human colon cancer," International Journal of Oncology, vol. 42, no. 2, pp. 676682, 2013.

[122] W. Wu, J. Yang, X. Feng et al., "MicroRNA-32 (miR-32) regulates phosphatase and tensin homologue (PTEN) expression and promotes growth, migration, and invasion in colorectal carcinoma cells," Molecular Cancer, vol. 12, article 30, 2013.

[123] G. Zhang, H. Zhou, H. Xiao, Z. Liu, H. Tian, and T. Zhou, "MicroRNA-92a functions as an oncogene in colorectal cancer by targeting PTEN," Digestive Diseases and Sciences, vol. 59, no. 1, pp. 98-107, 2014.

[124] Z. Huang, S. Huang, Q. Wang et al., "MicroRNA-95 promotes cell proliferation and targets sorting nexin 1 in human colorectal carcinoma," Cancer Research, vol. 71, no. 7, pp. 2582-2589, 2011.

[125] F. Gao and W. Wang, "MicroRNA-96 promotes the proliferation of colorectal cancer cells and targets tumor protein p53 inducible nuclear protein 1, forkhead box protein O1 (FOXO1) and FOXO3a," Molecular Medicine Reports, vol. 11, no. 2, pp. 1200-1206, 2015.

[126] L. Geng, B. Sun, B. Gao et al., "MicroRNA-103 promotes colorectal cancer by targeting tumor suppressor DICER and PTEN," International Journal of Molecular Sciences, vol. 15, no. 5, pp. 8458-8472, 2014.

[127] R. Nagel, C. Le Sage, B. Diosdado et al., "Regulation of the adenomatous polyposis coli gene by the miR-135 family in colorectal cancer," Cancer Research, vol. 68, no. 14, pp. 57955802, 2008.

[128] N. Valeri, P. Gasparini, M. Fabbri et al., "Modulation of mismatch repair and genomic stability by miR-155," Proceedings of the National Academy of Sciences of the United States of America, vol. 107, no. 15, pp. 6982-6987, 2010.

[129] D. Ji, Z. Chen, M. Li et al., "MicroRNA-181a promotes tumor growth and liver metastasis in colorectal cancer by targeting the tumor suppressor WIF-1," Molecular Cancer, vol. 13, article 86, 2014.

[130] Z. Wei, L. Cui, Z. Mei, M. Liu, and D. Zhang, "miR-181a mediates metabolic shift in colon cancer cells via the PTEN/AKT pathway," FEBS Letters, vol. 588, no. 9, pp. 1773-1779, 2014.

[131] Y. Zhang, X. Wang, Z. Wang, H. Tang, H. Fan, and Q. Guo, "miR-182 promotes cell growth and invasion by targeting forkhead box F2 transcription factor in colorectal cancer," Oncology Reports, vol. 33, no. 5, pp. 2592-2598, 2015. 
[132] J.-S. Mo, K. J. A. Alam, I.-H. Kang et al., "MicroRNA 196B regulates FAS-mediated apoptosis in colorectal cancer cells," Oncotarget, vol. 6, no. 5, pp. 2843-2855, 2015.

[133] C. Polytarchou, D. W. Hommes, T. Palumbo et al., "MicroRNA214 is associated with progression of ulcerative colitis, and inhibition reduces development of colitis and colitis-associated cancer in mice," Gastroenterology, vol. 149, no. 4, pp. 981-992.e11, 2015.

[134] D. Sun, C. Wang, S. Long et al., "C/EBP- $\beta$-activated microRNA223 promotes tumour growth through targeting RASAl in human colorectal cancer," British Journal of Cancer, vol. 112, no. 9, pp. 1491-1500, 2015.

[135] H. Ling, K. Pickard, C. Ivan et al., "The clinical and biological significance of MIR-224 expression in colorectal cancer metastasis," Gut, vol. 65, no. 6, pp. 977-989, 2016.

[136] T. Suto, T. Yokobori, R. Yajima et al., "MicroRNA-7 expression in colorectal cancer is associated with poor prognosis and regulates cetuximab sensitivity via EGFR regulation," Carcinogenesis, vol. 36, no. 3, pp. 338-345, 2015.

[137] W. P. Tsang and T. T. Kwok, "The miR-18a* microRNA functions as a potential tumor suppressor by targeting on K-Ras," Carcinogenesis, vol. 30, no. 6, pp. 953-959, 2009.

[138] Y.-L. Ma, P. Zhang, F. Wang et al., "Human embryonic stem cells and metastatic colorectal cancer cells shared the common endogenous human microRNA-26b," Journal of Cellular and Molecular Medicine, vol. 15, no. 9, pp. 1941-1954, 2011.

[139] J. Ye, X. Wu, D. Wu et al., "miRNA-27b targets vascular endothelial growth factor $\mathrm{C}$ to inhibit tumor progression and angiogenesis in colorectal cancer," PLoS ONE, vol. 8, no. 4, Article ID e60687, 2013.

[140] M. Yamakuchi, M. Ferlito, and C. J. Lowenstein, "miR-34a repression of SIRT1 regulates apoptosis," Proceedings of the National Academy of Sciences of the United States of America, vol. 105, no. 36, pp. 13421-13426, 2008.

[141] H. Peng, J. Luo, H. Hao et al., "MicroRNA-100 regulates SW620 colorectal cancer cell proliferation and invasion by targeting RAP1B," Oncology Reports, vol. 31, no. 5, pp. 2055-2062, 2014.

[142] M.-B. Chen, L. Yang, P.-H. Lu et al., "MicroRNA-101 downregulates sphingosine kinase 1 in colorectal cancer cells," Biochemical and Biophysical Research Communications, vol. 463, no. 4, pp. 954-960, 2015.

[143] J. Zhang, Y. Lu, X. Yue et al., "MiR-124 suppresses growth of human colorectal cancer by inhibiting STAT3," PLOS ONE, vol. 8, no. 8, Article ID e70300, 2013.

[144] V. Stiegelbauer, S. Perakis, A. Deutsch, H. Ling, A. Gerger, and M. Pichler, "MicroRNAs as novel predictive biomarkers and therapeutic targets in colorectal cancer," World Journal of Gastroenterology, vol. 20, no. 33, pp. 11727-11735, 2014.

[145] Y. Liu, Y. Zhou, X. Feng et al., "MicroRNA-126 functions as a tumor suppressor in colorectal cancer cells by targeting CXCR4 via the AKT and ERK1/2 signaling pathways," International Journal of Oncology, vol. 44, no. 1, pp. 203-210, 2014.

[146] Y. Zhou, X. Feng, Y.-L. Liu et al., "Down-regulation of miR-126 is associated with colorectal cancer cells proliferation, migration and invasion by targeting IRS- 1 via the AKT and ERK1/2 signaling pathways," PLoS ONE, vol. 8, no. 11, Article ID e81203, 2013.

[147] H. Wang, H. An, B. Wang et al., "miR-133a represses tumour growth and metastasis in colorectal cancer by targeting LIM and $\mathrm{SH} 3$ protein 1 and inhibiting the MAPK pathway," European Journal of Cancer, vol. 49, no. 18, pp. 3924-3935, 2013.
[148] K. Zheng, W. Liu, Y. Liu, C. Jiang, and Q. Qian, "MicroRNA-133a suppresses colorectal cancer cell invasion by targeting fascinl," Oncology Letters, vol. 9, no. 2, pp. 869-874, 2015.

[149] F.-T. Duan, F. Qian, K. Fang, K.-Y. Lin, W.-T. Wang, and Y.Q. Chen, "miR-133b, a muscle-specific microRNA, is a novel prognostic marker that participates in the progression of human colorectal cancer via regulation of CXCR4 expression," Molecular Cancer, vol. 12, article 164, 2013.

[150] K.-M. Xiang and X.-R. Li, "MiR-133b acts as a tumor suppressor and negatively regulates TBPL1 in colorectal cancer cells," Asian Pacific Journal of Cancer Prevention, vol. 15, no. 8, pp. 3767-3772, 2014.

[151] K. Shen, Q. Liang, K. Xu et al., "MiR-139 inhibits invasion and metastasis of colorectal cancer by targeting the type I insulinlike growth factor receptor," Biochemical Pharmacology, vol. 84, no. 3, pp. 320-330, 2012.

[152] L. Zhang, Y. Dong, N. Zhu et al., "microRNA-139-5p exerts tumor suppressor function by targeting NOTCH1 in colorectal cancer," Molecular Cancer, vol. 13, article 124, 2014.

[153] J. Pekow, K. Meckel, U. Dougherty et al., “Tumor suppressors miR-143 and miR-145 and predicted target proteins API5, ERK5, K-RAS, and IRS-1 are differentially expressed in proximal and distal colon," American Journal of Physiology-Gastrointestinal and Liver Physiology, vol. 308, no. 3, pp. G179-G187, 2015.

[154] J. Su, H. Liang, W. Yao et al., "MiR-143 and MiR-145 regulate IGF1R to suppress cell proliferation in colorectal cancer," PLoS ONE, vol. 9, no. 12, Article ID el14420, 2014.

[155] T. Iwaya, T. Yokobori, N. Nishida et al., "Downregulation of miR-144 is associated with colorectal cancer progression via activation of mTOR signaling pathway," Carcinogenesis, vol. 33, no. 12, pp. 2391-2397, 2012.

[156] Y. Yin, Z.-P. Yan, N.-N. Lu et al., "Downregulation of miR-145 associated with cancer progression and VEGF transcriptional activation by targeting N-RAS and IRS1," Biochimica et Biophysica Acta (BBA)_Gene Regulatory Mechanisms, vol. 1829, no. 2, pp. 239-247, 2013.

[157] Y. Song, Y. Xu, Z. Wang et al., "MicroRNA-148b suppresses cell growth by targeting cholecystokinin-2 receptor in colorectal cancer," International Journal of Cancer, vol. 131, no. 5, pp. 10421051, 2012.

[158] G. Wang, X. Cao, S. Lai et al., "Altered p53 regulation of MIR$148 \mathrm{~b}$ and $\mathrm{p} 55 \mathrm{PIK}$ contributes to tumor progression in colorectal cancer," Oncogene, vol. 34, no. 7, pp. 912-921, 2015.

[159] B. Wang, Z.-L. Shen, Z.-D. Gao et al., "MiR-194, commonly repressed in colorectal cancer, suppresses tumor growth by regulating the MAP4K4/c-Jun/MDM2 signaling pathway," Cell Cycle, vol. 14, no. 7, pp. 1046-1058, 2015.

[160] H.-J. Zhao, L.-L. Ren, Z.-H. Wang et al., "MiR-194 deregulation contributes to colorectal carcinogenesis via targeting AKT2 pathway," Theranostics, vol. 4, no. 12, pp. 1193-1208, 2014.

[161] L. Liu, L. Chen, Y. Xu, R. Li, and X. Du, "microRNA-195 promotes apoptosis and suppresses tumorigenicity of human colorectal cancer cells," Biochemical and Biophysical Research Communications, vol. 400, no. 2, pp. 236-240, 2010.

[162] D. J. Jin, Y. T. Fang, Z. G. Li, Z. Chen, and J. B. Xiang, "Epithelial-mesenchymal transition-associated microRNAs in colorectal cancer and drug-targeted therapies (Review)," Oncology Reports, vol. 33, no. 2, pp. 515-525, 2015.

[163] X.-W. Wang, X.-Q. Xi, J. Wu, Y.-Y. Wan, H.-X. Hui, and X.F. Cao, "MicroRNA-206 attenuates tumor proliferation and 
migration involving the downregulation of NOTCH3 in colorectal cancer," Oncology Reports, vol. 33, no. 3, pp. 1402-1410, 2015.

[164] D.-L. Chen, Z.-Q. Wang, Z.-L. Zeng et al., "Identification of microRNA-214 as a negative regulator of colorectal cancer liver metastasis by way of regulation of fibroblast growth factor receptor 1 expression," Hepatology, vol. 60, no. 2, pp. 598-609, 2014.

[165] X. He, Y. Dong, C. W. Wu et al., "MicroRNA-218 inhibits cell cycle progression and promotes apoptosis in colon cancer by downregulating BMIl polycomb ring finger oncogene," Molecular Medicine, vol. 18, no. 12, pp. 1491-1498, 2012.

[166] T.-W. Ke, H.-L. Hsu, Y.-H. Wu, W. T.-L. Chen, Y.-W. Cheng, and C.-W. Cheng, "MicroRNA-224 suppresses colorectal cancer cell migration by targeting Cdc42," Disease Markers, vol. 2014, Article ID 617150, 11 pages, 2014.

[167] J.-Y. Sun, Y. Huang, J.-P. Li et al., “MicroRNA-320a suppresses human colon cancer cell proliferation by directly targeting $\beta$ catenin," Biochemical and Biophysical Research Communications, vol. 420, no. 4, pp. 787-792, 2012.

[168] H. Zhao, T. Dong, H. Zhou et al., "miR-320a suppresses colorectal cancer progression by targeting Racl," Carcinogenesis, vol. 35, no. 4, pp. 886-895, 2014.

[169] H. Wang, J. Wu, X. Meng et al., "Microrna-342 inhibits colorectal cancer cell proliferation and invasion by directly targeting dna methyltransferase 1," Carcinogenesis, vol. 32, no. 7, pp. 10331042, 2011.

[170] J. Nie, L. Liu, W. Zheng et al., "microRNA-365, down-regulated in colon cancer, inhibits cell cycle progression and promotes apoptosis of colon cancer cells by probably targeting Cyclin D1 and Bcl-2," Carcinogenesis, vol. 33, no. 1, pp. 220-225, 2012.

[171] Y. Wang, Q. Tang, M. Li, S. Jiang, and X. Wang, "MicroRNA375 inhibits colorectal cancer growth by targeting PIK3CA," Biochemical and Biophysical Research Communications, vol. 444, no. 2, pp. 199-204, 2014.

[172] G.-J. Zhang, H. Zhou, H.-X. Xiao, Y. Li, and T. Zhou, "MiR-378 is an independent prognostic factor and inhibits cell growth and invasion in colorectal cancer," BMC Cancer, vol. 14, article 109, 2014.

[173] Y. Sun, S. Shen, X. Liu et al., "MiR-429 inhibits cells growth and invasion and regulates EMT-related marker genes by targeting Onecut2 in colorectal carcinoma," Molecular and Cellular Biochemistry, vol. 390, no. 1-2, pp. 19-30, 2014.

[174] J. Chai, S. Wang, D. Han, W. Dong, C. Xie, and H. Guo, "MicroRNA-455 inhibits proliferation and invasion of colorectal cancer by targeting RAF proto-oncogene serine/threonineprotein kinase," Tumor Biology, vol. 36, no. 2, pp. 1313-1321, 2015.

[175] H. Nakano, T. Miyazawa, K. Kinoshita, Y. Yamada, and T. Yoshida, "Functional screening identifies a microRNA, miR491 that induces apoptosis by targeting Bcl-X(L) in colorectal cancer cells," International Journal of Cancer, vol. 127, no. 5, pp. 1072-1080, 2010.

[176] K. Ma, X. Pan, P. Fan et al., "Loss of miR-638 in vitro promotes cell invasion and a mesenchymal-like transition by influencing SOX2 expression in colorectal carcinoma cells," Molecular Cancer, vol. 13, article 118, 2014.

[177] G. Nakajima, K. Hayashi, Y. Xi et al., "Non-coding microRNAs hsa-let-7g and hsa-miR-181b are associated with chemoresponse to S-1 in colon cancer," Cancer Genomics and Proteomics, vol. 3, no. 5, pp. 317-324, 2006.

[178] N. Nishida, S. Yamashita, K. Mimori et al., "MicroRNA-10b is a prognostic indicator in colorectal cancer and confers resistance to the chemotherapeutic agent 5-fluorouracil in colorectal cancer cells," Annals of Surgical Oncology, vol. 19, no. 9, pp. 3065-3071, 2012.

[179] K. Kurokawa, T. Tanahashi, T. Iima et al., "Role of miR-19b and its target mRNAs in 5-fluorouracil resistance in colon cancer cells," Journal of Gastroenterology, vol. 47, no. 8, pp. 883-895, 2012.

[180] H. Chai, M. Liu, R. Tian, X. Li, and H. Tang, "miR-20a targets BNIP2 and contributes chemotherapeutic resistance in colorectal adenocarcinoma SW480 and SW620 cell lines," Acta Biochimica et Biophysica Sinica, vol. 43, no. 3, pp. 217-225, 2011.

[181] J. Shang, F. Yang, Y. Wang et al., "MicroRNA-23a antisense enhances 5-fluorouracil chemosensitivity through apaf1/caspase-9 apoptotic pathway in colorectal cancer cells," Journal of Cellular Biochemistry, vol. 115, no. 4, pp. 772-784, 2014.

[182] C.-J. Wang, J. Stratmann, Z.-G. Zhou, and X.-F. Sun, "Suppression of microRNA-31 increases sensitivity to 5-FU at an early stage, and affects cell migration and invasion in HCT-116 colon cancer cells," BMC Cancer, vol. 10, article 616, 2010.

[183] B. Song, Y. Wang, Y. Xi et al., "Mechanism of chemoresistance mediated by miR-140 in human osteosarcoma and colon cancer cells," Oncogene, vol. 28, no. 46, pp. 4065-4074, 2009.

[184] M. Takahashi, M. Cuatrecasas, F. Balaguer et al., "The clinical significance of MiR-148a as a predictive biomarker in patients with advanced colorectal cancer," PLoS ONE, vol. 7, no. 10, Article ID e46684, 2012.

[185] B. Song, Y. Wang, M. A. Titmus et al., "Molecular mechanism of chemoresistance by miR-215 in osteosarcoma and colon cancer cells," Molecular Cancer, vol. 9, article 96, 2010.

[186] V. Boni, N. Bitarte, I. Cristobal et al., "miR-192/miR-215 influence 5-fluorouracil resistance through cell cycle-mediated mechanisms complementary to its post-transcriptional thymidilate synthase regulation," Molecular Cancer Therapeutics, vol. 9, no. 8, pp. 2265-2275, 2010.

[187] Y. Zhou, G. Wan, R. Spizzo et al., "miR-203 induces oxaliplatin resistance in colorectal cancer cells by negatively regulating ATM kinase," Molecular Oncology, vol. 8, no. 1, pp. 83-92, 2014.

[188] K. Xu, X. Liang, K. Shen et al., "MiR-222 modulates multidrug resistance in human colorectal carcinoma by down-regulating ADAM-17," Experimental Cell Research, vol. 318, no. 17, pp. 21682177, 2012.

[189] Y. Zhang, L. Geng, G. Talmon, and J. Wang, "MicroRNA$520 \mathrm{~g}$ confers drug resistance by regulating p21 expression in colorectal cancer," The Journal of Biological Chemistry, vol. 290, no. 10, pp. 6215-6225, 2015.

[190] M. H. Rasmussen, N. F. Jensen, L. S. Tarpgaard et al., "High expression of microRNA-625-3p is associated with poor response to first-line oxaliplatin based treatment of metastatic colorectal cancer," Molecular Oncology, vol. 7, no. 3, pp. 637-646, 2013.

[191] H. Zhang, J. Tang, C. Li et al., "MiR-22 regulates 5-FU sensitivity by inhibiting autophagy and promoting apoptosis in colorectal cancer cells," Cancer Letters, vol. 356, no. 2, pp. 781-790, 2015.

[192] Y. Akao, F. Khoo, M. Kumazaki, H. Shinohara, K. Miki, and N. Yamada, "Extracellular disposal of tumor-suppressor miRs-145 and -34a via microvesicles and 5-FU resistance of human colon cancer cells," International Journal of Molecular Sciences, vol. 15, no. 1, pp. 1392-1401, 2014.

[193] X. Li, H. Zhao, X. Zhou, and L. Song, "Inhibition of lactate dehydrogenase A by microRNA-34a resensitizes colon cancer cells to 5-fluorouracil," Molecular Medicine Reports, vol. 11, no. 1, pp. 577-582, 2015. 
[194] H. Siemens, R. Jackstadt, M. Kaller, and H. Hermeking, "Repression of c-Kit by p53 is mediated by miR-34 and is associated with reduced chemoresistance, migration and stemness," Oncotarget, vol. 4, no. 9, pp. 1399-1415, 2013.

[195] J. He, G. Xie, J. Tong et al., "Overexpression of microRNA-122 re-sensitizes 5-FU-resistant colon cancer cells to 5-FU through the inhibition of PKM2 in vitro and in vivo," Cell Biochemistry and Biophysics, vol. 70, no. 2, pp. 1343-1350, 2014.

[196] M. Karaayvaz, H. Zhai, and J. Ju, "miR-129 promotes apoptosis and enhances chemosensitivity to 5-fluorouracil in colorectal cancer," Cell Death and Disease, vol. 4, no. 6, article e659, 2013.

[197] Y. Dong, J. Zhao, C.-W. Wu et al., "Tumor suppressor functions of miR-133a in colorectal cancer," Molecular Cancer Research, vol. 11, no. 9, pp. 1051-1060, 2013.

[198] H. Liu, Y. Yin, Y. Hu et al., "miR-139-5p sensitizes colorectal cancer cells to 5-fluorouracil by targeting NOTCH-1," Pathology Research and Practice, vol. 212, no. 7, pp. 643-649, 2016.

[199] S. Tanaka, M. Hosokawa, T. Yonezawa, W. Hayashi, K. Ueda, and S. Iwakawa, "Induction of epithelial-mesenchymal transition and down-regulation of MIR-200c and MIR-141 in oxaliplatinresistant colorectal cancer cells," Biological and Pharmaceutical Bulletin, vol. 38, no. 3, pp. 435-440, 2015.

[200] P. M. Borralho, B. T. Kren, R. E. Castro, I. B. Moreira da Silva, C. J. Steer, and C. M. P. Rodrigues, "MicroRNA-143 reduces viability and increases sensitivity to 5-fluorouracil in HCT116 human colorectal cancer cells," FEBS Journal, vol. 276, no. 22, pp. 6689-6700, 2009.

[201] X. Qian, J. Yu, Y. Yin et al., "MicroRNA-143 inhibits tumor growth and angiogenesis and sensitizes chemosensitivity to oxaliplatin in colorectal cancers," Cell Cycle, vol. 12, no. 9, pp. 1385-1394, 2013.

[202] R. L. Liu, Y. Dong, Y. Z. Deng, W. J. Wang, and W. D. Li, "Tumor suppressor miR-145 reverses drug resistance by directly targeting DNA damage-related gene RAD18 in colorectal cancer," Tumor Biology, vol. 36, no. 7, pp. 5011-5019, 2015.

[203] J. Zhang, H. Guo, H. Zhang et al., "Putative tumor suppressor miR-145 inhibits colon cancer cell growth by targeting oncogene friend leukemia virus integration 1 gene," Cancer, vol. 117, no. 1, pp. 86-95, 2011.

[204] X. Liu, T. Xie, X. Mao, L. Xue, X. Chu, and L. Chen, "MicroRNA149 increases the sensitivity of colorectal cancer cells to 5fluorouracil by targeting forkhead box transcription factor FOXM1," Cellular Physiology and Biochemistry, vol. 39, no. 2, pp. 617-629, 2016.

[205] L. Zhang, K. Pickard, V. Jenei et al., "miR-153 supports colorectal cancer progression via pleiotropic effects that enhance invasion and chemotherapeutic resistance," Cancer Research, vol. 73, no. 21, pp. 6435-6447, 2013.

[206] C. C. Schimanski, K. Frerichs, F. Rahman et al., "High miR-196a levels promote the oncogenic phenotype of colorectal cancer cells," World Journal of Gastroenterology, vol. 15, no. 17, pp. 20892096, 2009.

[207] D. Senfter, S. Holzner, M. Kalipciyan et al., "Loss of miR-200 family in 5-fluorouracil resistant colon cancer drives lymphendothelial invasiveness in vitro," Human Molecular Genetics, vol. 24, no. 13, pp. 3689-3698, 2015.

[208] T. Li, F. Gao, and X.-P. Zhang, "miR-203 enhances chemosensitivity to 5-fluorouracil by targeting thymidylate synthase in colorectal cancer," Oncology Reports, vol. 33, no. 2, pp. 607-614, 2015.
[209] H. Wu, Y. Liang, L. Shen, and L. Shen, "MicroRNA-204 modulates colorectal cancer cell sensitivity in response to 5fluorouracil-based treatment by targeting high mobility group protein A2," Biology Open, vol. 5, no. 5, pp. 563-570, 2016.

[210] K. Xu, X. Liang, K. Shen et al., "miR-297 modulates multidrug resistance in human colorectal carcinoma by down-regulating MRP-2," Biochemical Journal, vol. 446, no. 2, pp. 291-300, 2012.

[211] N. Bitarte, E. Bandres, V. Boni et al., "MicroRNA-451 is involved in the self-renewal, tumorigenicity, and chemoresistance of colorectal cancer stem cells," STEM CELLS, vol. 29, no. 11, pp. 1661-1671, 2011.

[212] S. T. Guo, C. C. Jiang, G. P. Wang et al., "MicroRNA-497 targets insulin-like growth factor 1 receptor and has a tumour suppressive role in human colorectal cancer," Oncogene, vol. 32, no. 15, pp. 1910-1920, 2013.

[213] K. K. W. To, W. W. Leung, and S. S. M. Ng, "Exploiting a novel miR-519c-HuR-ABCG2 regulatory pathway to overcome chemoresistance in colorectal cancer," Experimental Cell Research, vol. 338, no. 2, pp. 222-231, 2015.

[214] K. Xu, X. Liang, D. Cui, Y. Wu, W. Shi, and J. Liu, “miR-1915 inhibits Bcl-2 to modulate multidrug resistance by increasing drug-sensitivity in human colorectal carcinoma cells," Molecular Carcinogenesis, vol. 52, no. 1, pp. 70-78, 2013.

[215] M. Ragusa, A. Majorana, L. Statello et al., "Specific alterations of microRNA transcriptome and global network structure in colorectal carcinoma after cetuximab treatment," Molecular Cancer Therapeutics, vol. 9, no. 12, pp. 3396-3409, 2010.

[216] J. Mlcochova, P. Faltejskova-Vychytilova, M. Ferracin et al., "MicroRNA expression profiling identifies miR-31-5p/3p as associated with time to progression in wild-type RAS metastatic colorectal cancer treated with cetuximab," Oncotarget, vol. 6, no. 36, pp. 38695-38704, 2015.

[217] T. F. Hansen, A. L. Carlsen, N. H. H. Heegaard, F. B. Sørensen, and A. Jakobsen, "Changes in circulating microRNA-126 during treatment with chemotherapy and bevacizumab predicts treatment response in patients with metastatic colorectal cancer," British Journal of Cancer, vol. 112, no. 4, pp. 624-629, 2015.

[218] P. Mussnich, R. Rosa, R. Bianco, A. Fusco, and D. D’Angelo, "MiR-199a-5p and miR-375 affect colon cancer cell sensitivity to cetuximab by targeting PHLPP1," Expert Opinion on Therapeutic Targets, vol. 19, no. 8, pp. 1017-1026, 2015.

[219] W. Zhang, T. Winder, Y. Ning et al., "A let-7 microRNA-binding site polymorphism in $3^{\prime}$-untranslated region of KRAS gene predicts response in wild-type KRAS patients with metastatic colorectal cancer treated with cetuximab monotherapy," Annals of Oncology, vol. 22, no. 1, pp. 104-109, 2011.

[220] A. Hata and J. Lieberman, "Dysregulation of microRNA biogenesis and gene silencing in cancer," Science Signaling, vol. 8, no. 368 , p. re3, 2015

[221] C. Faber, T. Kirchner, and F. Hlubek, "The impact of microRNAs on colorectal cancer," Virchows Archiv, vol. 454, no. 4, pp. 359$367,2009$.

[222] M. L. Slattery, E. Wolff, M. D. Hoffman, D. F. Pellatt, B. Milash, and R. K. Wolff, "MicroRNAs and colon and rectal cancer: differential expression by tumor location and subtype," Genes Chromosomes and Cancer, vol. 50, no. 3, pp. 196-206, 2011.

[223] L. Yang, N. Belaguli, and D. H. Berger, "MicroRNA and colorectal cancer," World Journal of Surgery, vol. 33, no. 4, pp. 638-646, 2009.

[224] L. Cekaite, P. W. Eide, G. E. Lind, R. I. Skotheim, and R. A. Lothe, "MicroRNAs as growth regulators, their function and 
biomarker status in colorectal cancer," Oncotarget, vol. 7, no. 6, pp. 6476-6505, 2015.

[225] M. Liu and H. Chen, "The role of microRNAs in colorectal cancer," Journal of Genetics and Genomics, vol. 37, no. 6, pp. 347$358,2010$.

[226] A. Mohammadi, B. Mansoori, and B. Baradaran, "The role of microRNAs in colorectal cancer," Biomedicine \& Pharmacotherapy, vol. 84, pp. 705-713, 2016.

[227] S. Rossi, S. Kopetz, R. Davuluri, S. R. Hamilton, and G. A. Calin, "MicroRNAs, ultraconserved genes and colorectal cancers," International Journal of Biochemistry and Cell Biology, vol. 42, no. 8, pp. 1291-1297, 2010.

[228] O. Slaby, M. Svoboda, J. Michalek, and R. Vyzula, "MicroRNAs in colorectal cancer: translation of molecular biology into clinical application," Molecular Cancer, vol. 8, article 102, 2009.

[229] J. Thomas, M. Ohtsuka, M. Pichler, and H. Ling, "MicroRNAs: clinical relevance in colorectal cancer," International Journal of Molecular Sciences, vol. 16, no. 12, pp. 28063-28076, 2015.

[230] W. K. K. Wu, P. T. Y. Law, C. W. Lee et al., "MicroRNA in colorectal cancer: from benchtop to bedside," Carcinogenesis, vol. 32, no. 3, pp. 247-253, 2011.

[231] A. J. Schetter, S. Y. Leung, J. J. Sohn et al., "MicroRNA expression profiles associated with prognosis and therapeutic outcome in colon adenocarcinoma," The Journal of the American Medical Association, vol. 299, no. 4, pp. 425-436, 2008.

[232] X. Wu, X. Xu, S. Li et al., "Identification and validation of potential biomarkers for the detection of dysregulated microrna by QPCR in patients with colorectal adenocarcinoma," PLoS ONE, vol. 10, no. 3, Article ID e0120024, 2015.

[233] T. Schepeler, J. T. Reinert, M. S. Ostenfeld et al., "Diagnostic and prognostic microRNAs in stage II colon cancer," Cancer Research, vol. 68, no. 15, pp. 6416-6424, 2008.

[234] J. Hamfjord, A. M. Stangeland, T. Hughes et al., "Differential expression of miRNAs in colorectal cancer: comparison of paired tumor tissue and adjacent normal mucosa using highthroughput sequencing," PLoS ONE, vol. 7, no. 4, Article ID e34150, 2012.

[235] J. Gu, Y. Chen, H. Huang, L. Yin, Z. Xie, and M. Q. Zhang, "Gene module based regulator inference identifying miR-139 as a tumor suppressor in colorectal cancer," Molecular BioSystems, vol. 10, no. 12, pp. 3249-3254, 2014.

[236] F. L. Yong, C. W. Law, and C. W. Wang, "Potentiality of a triple microRNA classifier: MiR-193a-3p, miR-23a and miR-338-5p for early detection of colorectal cancer," BMC Cancer, vol. 13, article no. 280, 2013.

[237] A. J. Schetter, H. Okayama, and C. C. Harris, "The role of MicroRNAs in colorectal cancer," Cancer Journal, vol. 18, no. 3, pp. 244-252, 2012.

[238] A. Mamoori, V. Gopalan, R. A. Smith, and A. K.-Y. Lam, "Modulatory roles of microRNAs in the regulation of different signalling pathways in large bowel cancer stem cells," Biology of the Cell, vol. 108, no. 3, pp. 51-64, 2016.

[239] H. Hermeking, "p53 enters the MicroRNA world," Cancer Cell, vol. 12, no. 5, pp. 414-418, 2007.

[240] Y. Li, M. Lauriola, D. Kim et al., "Adenomatous polyposis coli (APC) regulates miR17-92 cluster through $\beta$-catenin pathway in colorectal cancer," Oncogene, vol. 35 , no. 35 , pp. 4558-4568, 2016.

[241] S. Roy, Y. Yu, S. B. Padhye, F. H. Sarkar, and A. P. N. Majumdar, "Difluorinated-curcumin (CDF) restores PTEN expression in colon cancer cells by down-regulating miR-21," PLOS ONE, vol. 8, no. 7, Article ID e68543, 2013.
[242] Y. Yu, P. Nangia-Makker, L. Farhana, S. G. Rajendra, E. Levi, and A. P. N. Majumdar, "miR-21 and miR-145 cooperation in regulation of colon cancer stem cells," Molecular Cancer, vol. 14, no. 1, article 98, 2015.

[243] A. Moustakas and C.-H. Heldin, “The regulation of TGF $\beta$ signal transduction," Development, vol. 136, no. 22, pp. 3699-3714, 2009.

[244] H. Zhang, W. Li, F. Nan et al., "MicroRNA expression profile of colon cancer stem-like cells in HT29 adenocarcinoma cell line," Biochemical and Biophysical Research Communications, vol. 404, no. 1, pp. 273-278, 2011.

[245] Y. Xi, R. Shalgi, O. Fodstad, Y. Pilpel, and J. Ju, "Differentially regulated micro-RNAs and actively translated messenger RNA transcripts by tumor suppressor p53 in colon cancer," Clinical Cancer Research, vol. 12, no. 7, part 1, pp. 2014-2024, 2006.

[246] V. Davalos and M. Esteller, "Unraveling the complex network of interactions between noncoding RNAs and epigenetics in cancer," Non-Coding RNAs and Cancer, pp. 125-148, 2014.

[247] D. Landi, F. Gemignani, and S. Landi, "Role of variations within microRNA-binding sites in cancer," Mutagenesis, vol. 27, no. 2, pp. 205-210, 2012.

[248] B. M. Ryan, A. I. Robles, and C. C. Harris, "Genetic variation in microRNA networks: the implications for cancer research," Nature Reviews Cancer, vol. 10, no. 6, pp. 389-402, 2010.

[249] R. Garzon, G. Marcucci, and C. M. Croce, "Targeting microRNAs in cancer: rationale, strategies and challenges," Nature Reviews Drug Discovery, vol. 9, no. 10, pp. 775-789, 2010.

[250] P. Vodicka, B. Pardini, V. Vymetalkova, and A. Naccarati, "Polymorphisms in non-coding RNA genes and their targets sites as risk factors of sporadic colorectal cancer," in Non-Coding RNAs in Colorectal Cancer, vol. 937 of Advances in Experimental Medicine and Biology, pp. 123-149, Springer International, Cham, Switzerland, 2016.

[251] X. M. Pan, X. Xiao, H. J. Qin et al., "MicroRNA variants and colorectal cancer risk: a meta-analysis," Genetics and Molecular Research, vol. 15, no. 3, 2016.

[252] L. Xu and W. Tang, "Associations of polymorphisms in mir196a2, mir-146a and mir-149 with colorectal cancer risk: a metaanalysis," Pathology and Oncology Research, vol. 22, no. 2, pp. 261-267, 2016.

[253] K. A. Zanetti, M. Haznadar, J. A. Welsh et al., "3/-UTR and functional secretor haplotypes in mannose-binding lectin 2 are associated with increased colon cancer risk in African Americans," Cancer Research, vol. 72, no. 6, pp. 1467-1477, 2012.

[254] D. Landi, F. Gemignani, A. Naccarati et al., "Polymorphisms within micro-RNA-binding sites and risk of sporadic colorectal cancer," Carcinogenesis, vol. 29, no. 3, pp. 579-584, 2008.

[255] B. W. Kang, H. S. Jeon, Y. S. Chae et al., "Impact of genetic variation in MicroRNA-binding site on susceptibility to colorectal cancer," Anticancer Research, vol. 36, no. 7, pp. 3353-3361, 2016.

[256] Y. Zhao, Y. Du, S. Zhao, and Z. Guo, "Single-nucleotide polymorphisms of microRNA processing machinery genes and risk of colorectal cancer," OncoTargets and Therapy, vol. 8, pp. 421-425, 2015.

[257] B. Pardini, F. Rosa, A. Naccarati et al., "Polymorphisms in microRNA genes as predictors of clinical outcomes in colorectal cancer patients," Carcinogenesis, vol. 36, no. 1, pp. 82-86, 2015.

[258] J. L. Rinn, M. Kertesz, J. K. Wang et al., "Functional demarcation of active and silent chromatin domains in human HOX loci by noncoding RNAs," Cell, vol. 129, no. 7, pp. 1311-1323, 2007. 
[259] R. A. Gupta, N. Shah, K. C. Wang et al., "Long non-coding RNA HOTAIR reprograms chromatin state to promote cancer metastasis," Nature, vol. 464, no. 7291, pp. 1071-1076, 2010.

[260] M. Cesana, D. Cacchiarelli, I. Legnini et al., "A long noncoding RNA controls muscle differentiation by functioning as a competing endogenous RNA," Cell, vol. 147, no. 2, pp. 358-369, 2011.

[261] D. W. Thomson and M. E. Dinger, "Endogenous microRNA sponges: evidence and controversy," Nature Reviews Genetics, vol. 17, no. 5, pp. 272-283, 2016.

[262] M. N. Rossi and F. Antonangeli, "LncRNAs: new players in apoptosis control," International Journal of Cell Biology, vol. 2014, Article ID 473857, 7 pages, 2014.

[263] E. Saus, A. Brunet-Vega, S. Iraola-Guzmán, C. Pegueroles, T. Gabaldón, and C. Pericay, "Long non-coding RNAs as potential novel prognostic biomarkers in colorectal cancer," Frontiers in Genetics, vol. 7, article no. 54, 2016.

[264] X. Xie, B. Tang, Y.-F. Xiao et al., "Long non-coding RNAs in colorectal cancer," Oncotarget, vol. 7, no. 5, pp. 5226-5239, 2016.

[265] O. Wapinski and H. Y. Chang, "Long noncoding RNAs and human disease," Trends in Cell Biology, vol. 21, no. 6, pp. 354361, 2011.

[266] J. R. Prensner and A. M. Chinnaiyan, "The emergence of lncRNAs in cancer biology," Cancer Discovery, vol. 1, no. 5, pp. 391-407, 2011.

[267] M. Ragusa, C. Barbagallo, L. Statello et al., "Non-coding landscapes of colorectal cancer," World Journal of Gastroenterology, vol. 21, no. 41, pp. 11709-11739, 2015.

[268] X. Huang, T. Yuan, M. Tschannen et al., "Characterization of human plasma-derived exosomal RNAs by deep sequencing," BMC Genomics, vol. 14, no. 1, article no. 319, 2013.

[269] J. Wang, X. Liu, H. Wu et al., "CREB up-regulates long non-coding RNA, HULC expression through interaction with microRNA-372 in liver cancer," Nucleic Acids Research, vol. 38, no. 16, pp. 5366-5383, 2010.

[270] I. J. Matouk, I. Abbasi, A. Hochberg, E. Galun, H. Dweik, and M. Akkawi, "Highly upregulated in liver cancer noncoding RNA is overexpressed in hepatic colorectal metastasis," European Journal of Gastroenterology and Hepatology, vol. 21, no. 6, pp. 688-692, 2009.

[271] K. Panzitt, M. M. O. Tschernatsch, C. Guelly et al., "Characterization of HULC, a novel gene with striking up-regulation in hepatocellular carcinoma, as noncoding RNA," Gastroenterology, vol. 132, no. 1, pp. 330-342, 2007.

[272] Q. Liu, J. Huang, N. Zhou et al., "LncRNA loc285194 is a p53regulated tumor suppressor," Nucleic Acids Research, vol. 41, no. 9, pp. 4976-4987, 2013.

[273] W.-C. Liang, W.-M. Fu, C.-W. Wong et al., "The LncRNA H19 promotes epithelial to mesenchymal transition by functioning as MiRNA sponges in colorectal cancer," Oncotarget, vol. 6, no. 26, pp. 22513-22525, 2015.

[274] A. Keniry, D. Oxley, P. Monnier et al., “The H19 lincRNA is a developmental reservoir of miR-675 that suppresses growth and Igflr," Nature Cell Biology, vol. 14, no. 7, pp. 659-665, 2012.

[275] X. Cai and B. R. Cullen, "The imprinted H19 noncoding RNA is a primary microRNA precursor," RNA, vol. 13, no. 3, pp. 313-316, 2007.

[276] W. P. Tsang, E. K. O. Ng, S. S. M. Ng et al., "Oncofetal H19derived miR-675 regulates tumor suppressor RB in human colorectal cancer," Carcinogenesis, vol. 31, no. 3, pp. 350-358, 2010.
[277] Q. Ji, L. Zhang, X. Liu et al., "Long non-coding RNA MALAT1 promotes tumour growth and metastasis in colorectal cancer through binding to SFPQ and releasing oncogene PTBP2 from SFPQ/PTBP2 complex," British Journal of Cancer, vol. 111, no. 4, pp. 736-748, 2014.

[278] L. D. Graham, S. K. Pedersen, G. S. Brown et al., "Colorectal Neoplasia Differentially Expressed (CRNDE), a Novel Gene with Elevated Expression in Colorectal Adenomas and Adenocarcinomas," Genes and Cancer, vol. 2, no. 8, pp. 829-840, 2011.

[279] M. Svoboda, J. Slyskova, M. Schneiderova et al., "HOTAIR long non-coding RNA is a negative prognostic factor not only in primary tumors, but also in the blood of colorectal cancer patients," Carcinogenesis, vol. 35, no. 7, pp. 1510-1515, 2014.

[280] B. C. Ellis, P. L. Molloy, and L. D. Graham, "CRNDE: a long non-coding RNA involved in CanceR Neurobiology, and DEvelopment," Frontiers in Genetics, vol. 3, article 270, 2012.

[281] M. Hajjari and A. Salavaty, "HOTAIR: an oncogenic long noncoding RNA in different cancers," Cancer Biology and Medicine, vol. 12, no. 1, pp. 1-9, 2015.

[282] X. Ge, Y. Chen, X. Liao et al., "Overexpression of long noncoding RNA PCAT-1 is a novel biomarker of poor prognosis in patients with colorectal cancer," Medical Oncology, vol. 30, no. 2, article 588, 2013.

[283] X. He, X. Tan, X. Wang et al., "C-Myc-activated long noncoding RNA CCAT1 promotes colon cancer cell proliferation and invasion," Tumor Biology, vol. 35, no. 12, pp. 12181-12188, 2014.

[284] H. Lee, C. Kim, J.-L. Ku et al., "A long non-coding RNA snaR contributes to 5 -fluorouracil resistance in human colon cancer cells," Molecules and Cells, vol. 37, no. 7, pp. 540-546, 2014.

[285] Z. Bian, L. Jin, J. Zhang et al., "LncRNA-UCAl enhances cell proliferation and 5-fluorouracil resistance in colorectal cancer by inhibiting miR-204-5p," Scientific Reports, vol. 6, article 23892, 2016.

[286] A. Diaz-Lagares, A. B. Crujeiras, P. Lopez-Serra et al., "Epigenetic inactivation of the p53-induced long noncoding RNA TP53 target 1 in human cancer," Proceedings of the National Academy of Sciences, vol. 113, no. 47, pp. E7535-E7544, 2016.

[287] G. Wang, Z. Li, Q. Zhao et al., "LincRNA-p21 enhances the sensitivity of radiotherapy for human colorectal cancer by targeting the Wnt/ $\beta$-catenin signaling pathway," Oncology Reports, vol. 31, no. 4, pp. 1839-1845, 2014.

[288] N. Dimitrova, J. R. Zamudio, R. M. Jong et al., "LincRNA-p21 activates $\mathrm{p} 21$ in cis to promote polycomb target gene expression and to enforce the G1/S checkpoint," Molecular Cell, vol. 54, no. 5, pp. 777-790, 2014.

[289] M.-T. Hsu and M. Coca-Prados, "Electron microscopic evidence for the circular form of RNA in the cytoplasm of eukaryotic cells," Nature, vol. 280, no. 5720, pp. 339-340, 1979.

[290] J. M. Nigro, K. R. Cho, E. R. Fearon et al., "Scrambled exons," Cell, vol. 64, no. 3, pp. 607-613, 1991.

[291] J. Li, J. Yang, P. Zhou et al., "Circular RNAs in cancer: novel insights into origins, properties, functions and implications," American Journal of Cancer Research, vol. 5, no. 2, pp. 472-480, 2015.

[292] Y. Zhang, X.-O. Zhang, T. Chen et al., "Circular intronic long noncoding RNAs," Molecular Cell, vol. 51, no. 6, pp. 792-806, 2013.

[293] A. Bachmayr-Heyda, A. T. Reiner, K. Auer et al., "Correlation of circular RNA abundance with proliferation-exemplified with colorectal and ovarian cancer, idiopathic lung fibrosis, and normal human tissues," Scientific Reports, vol. 5, article 8057, 2015. 
[294] Y. Li, Q. Zheng, C. Bao et al., "Circular RNA is enriched and stable in exosomes: a promising biomarker for cancer diagnosis," Cell Research, vol. 25, no. 8, pp. 981-984, 2015.

[295] F. Wang, A. J. Nazarali, and S. Ji, "Circular RNAs as potential biomarkers for cancer diagnosis and therapy," American Journal of Cancer Research, vol. 6, no. 6, pp. 1167-1176, 2016.

[296] S. Memczak, M. Jens, A. Elefsinioti et al., "Circular RNAs are a large class of animal RNAs with regulatory potency," Nature, vol. 495, no. 7441, pp. 333-338, 2013.

[297] T. B. Hansen, T. I. Jensen, B. H. Clausen et al., "Natural RNA circles function as efficient microRNA sponges," Nature, vol. 495, no. 7441, pp. 384-388, 2013.

[298] N. Zhang, X. Li, C. W. Wu et al., "MicroRNA-7 is a novel inhibitor of YY1 contributing to colorectal tumorigenesis," Oncogene, vol. 32, no. 42, pp. 5078-5088, 2013.

[299] L. Peng, X. Q. Yuan, and G. C. Li, "The emerging landscape of circular RNA ciRS-7 in cancer (Review)," Oncology Reports, vol. 33, no. 6, pp. 2669-2674, 2015.

[300] W. R. Jeck and N. E. Sharpless, "Detecting and characterizing circular RNAs," Nature Biotechnology, vol. 32, no. 5, pp. 453-461, 2014.

[301] J. Guarnerio, M. Bezzi, J. C. Jeong et al., "Oncogenic role of fusion-circRNAs derived from cancer-associated chromosomal translocations," Cell, vol. 165, no. 2, pp. 289-302, 2016.

[302] P. Piedbois, P. Rougier, M. Buyse et al., "Efficacy of intravenous continuous infusion of fluorouracil compared with bolus administration in advanced colorectal cancer," Journal of Clinical Oncology, vol. 16, no. 1, pp. 301-308, 1998.

[303] B. Gustavsson, G. Carlsson, D. MacHover et al., "A review of the evolution of systemic chemotherapy in the management of colorectal cancer," Clinical Colorectal Cancer, vol. 14, no. 1, pp. 1-10, 2015.

[304] V. Heinemann, L. F. von Weikersthal, T. Decker et al., "FOLFIRI plus cetuximab versus FOLFIRI plus bevacizumab as firstline treatment for patients with metastatic colorectal cancer (FIRE-3): a randomised, open-label, phase 3 trial," The Lancet Oncology, vol. 15, no. 10, pp. 1065-1075, 2014.

[305] F. F. Kabbinavar, J. Hambleton, R. D. Mass, H. I. Hurwitz, E. Bergsland, and S. Sarkar, "Combined analysis of efficacy: the addition of bevacizumab to fluorouracil/leucovorin improves survival for patients with metastatic colorectal cancer," Journal of Clinical Oncology, vol. 23, no. 16, pp. 3706-3712, 2005.

[306] S.-Y. Lee and S. C. Oh, "Advances of targeted therapy in treatment of unresectable metastatic colorectal cancer," BioMed Research International, vol. 2016, Article ID 7590245, 14 pages, 2016.

[307] E. Van Cutsem, C.-H. Köhne, I. Láng et al., "Cetuximab plus irinotecan, fluorouracil, and leucovorin as first-line treatment for metastatic colorectal cancer: updated analysis of overall survival according to tumor KRAS and BRAF mutation status," Journal of Clinical Oncology, vol. 29, no. 15, pp. 2011-2019, 2011.

[308] F. Ciardiello and G. Tortora, "EGFR antagonists in cancer treatment," New England Journal of Medicine, vol. 358, no. 11, pp. 1096-1174, 2008.

[309] D. Rodrigues, A. Longatto-Filho, and S. F. Martins, "Predictive biomarkers in colorectal cancer: from the single therapeutic target to a plethora of options," BioMed Research International, vol. 2016, Article ID 6896024, 12 pages, 2016.

[310] R. G. Amado, M. Wolf, M. Peeters et al., "Wild-type KRAS is required for panitumumab efficacy in patients with metastatic colorectal cancer," Journal of Clinical Oncology, vol. 26, no. 10, pp. 1626-1634, 2008.
[311] C. Bokemeyer, I. Bondarenko, A. Makhson et al., "Fluorouracil, leucovorin, and oxaliplatin with and without cetuximab in the first-line treatment of metastatic colorectal cancer," Journal of Clinical Oncology, vol. 27, no. 5, pp. 663-671, 2009.

[312] C. S. Karapetis, S. Khambata-Ford, D. J. Jonker et al., "K-ras mutations and benefit from cetuximab in advanced colorectal cancer," The New England Journal of Medicine, vol. 359, no. 17, pp. 1757-1765, 2008.

[313] J.-Y. Douillard, K. S. Oliner, S. Siena et al., "PanitumumabFOLFOX4 treatment and RAS mutations in colorectal cancer," The New England Journal of Medicine, vol. 369, no. 11, pp. 10231034, 2013.

[314] T. S. Maughan, R. A. Adams, C. G. Smith et al., "Addition of cetuximab to oxaliplatin-based first-line combination chemotherapy for treatment of advanced colorectal cancer: results of the randomised phase $3 \mathrm{MRC}$ COIN trial," The Lancet, vol. 377, no. 9783, pp. 2103-2114, 2011.

[315] M. V. Blagosklonny, "How Avastin potentiates chemotherapeutic drugs: action and reaction in antiangiogenic therapy," Cancer Biology and Therapy, vol. 4, no. 12, pp. 1307-1310, 2005.

[316] M. A. Bruhn, A. R. Townsend, C. Khoon Lee et al., "Proangiogenic tumor proteins as potential predictive or prognostic biomarkers for bevacizumab therapy in metastatic colorectal cancer," International Journal of Cancer, vol. 135, no. 3, pp. 731741, 2014

[317] H. Hayashi, T. Arao, K. Matsumoto et al., "Biomarkers of reactive resistance and early disease progression during chemotherapy plus bevacizumab treatment for colorectal carcinoma," Oncotarget, vol. 5, no. 9, pp. 2588-2595, 2014.

[318] F. Loupakis, C. Cremolini, A. Fioravanti et al., "Pharmacodynamic and pharmacogenetic angiogenesis-related markers of first-line FOLFOXIRI plus bevacizumab schedule in metastatic colorectal cancer," British Journal of Cancer, vol. 104, no. 8, pp. 1262-1269, 2011.

[319] M. Scartozzi, L. Vincent, M. Chiron, and S. Cascinu, "Aflibercept, a new way to target angiogenesis in the second line treatment of metastatic colorectal cancer (mCRC)," Targeted Oncology, vol. 11, no. 4, pp. 489-500, 2016.

[320] R. Obermannová, E. Van Cutsem, T. Yoshino et al., "Subgroup analysis in RAISE: a randomized, double-blind phase III study of irinotecan, folinic acid, and 5-fluorouracil (FOLFIRI) plus ramucirumab or placebo in patients with metastatic colorectal carcinoma progression," Annals of Oncology, vol. 27, no. 11, pp. 2082-2090, 2016.

[321] Y. Ohhara, N. Fukuda, S. Takeuchi et al., "Role of targeted therapy in metastatic colorectal cancer," World Journal of Gastrointestinal Oncology, vol. 8, no. 9, pp. 642-655, 2016.

[322] A. Sanchez-Gastaldo, R. Gonzalez-Exposito, and R. GarciaCarbonero, "Ramucirumab clinical development: an emerging role in gastrointestinal tumors," Targeted Oncology, vol. 11, no. 4, pp. 479-487, 2016.

[323] S. M. Wilhelm, J. Dumas, L. Adnane et al., "Regorafenib (BAY 73-4506): a new oral multikinase inhibitor of angiogenic, stromal and oncogenic receptor tyrosine kinases with potent preclinical antitumor activity," International Journal of Cancer, vol. 129, no. 1, pp. 245-255, 2011.

[324] J. Li, S. Qin, R. Xu et al., "Regorafenib plus best supportive care versus placebo plus best supportive care in Asian patients with previously treated metastatic colorectal cancer (CONCUR): a randomised, double-blind, placebo-controlled, phase 3 trial," The Lancet Oncology, vol. 16, no. 6, pp. 619-629, 2015. 
[325] C. Lo Nigro, V. Ricci, D. Vivenza et al., "Prognostic and predictive biomarkers in metastatic colorectal cancer antiEGFR therapy," World Journal of Gastroenterology, vol. 22, no. 30, pp. 6944-6954, 2016.

[326] J.-S. Kim, C. Lee, C. L. Bonifant, H. Ressom, and T. Waldman, "Activation of p53-dependent growth suppression in human cells by mutations in PTEN or PIK3CA," Molecular and Cellular Biology, vol. 27, no. 2, pp. 662-677, 2007.

[327] A. Sartore-Bianchi, M. Martini, F. Molinari et al., "PIK3CA mutations in colorectal cancer are associated with clinical resistance to EGFR-targeted monoclonal antibodies," Cancer Research, vol. 69, no. 5, pp. 1851-1857, 2009.

[328] K. Lee and L. R. Ferguson, "MicroRNA biomarkers predicting risk, initiation and progression of colorectal cancer," World Journal of Gastroenterology, vol. 22, no. 33, pp. 7389-7401, 2016.

[329] Z. Li and T. M. Rana, "Therapeutic targeting of microRNAs: current status and future challenges," Nature Reviews Drug Discovery, vol. 13, no. 8, pp. 622-638, 2014.

[330] R. Yi, Y. Li, F. L. Wang, G. Miao, R. M. Qi, and Y. Y. Zhao, "MicroRNAs as diagnostic and prognostic biomarkers in colorectal cancer," World Journal of Gastrointestinal Oncology, vol. 8, no. 4, pp. 330-340, 2016.

[331] X. Li, J. Nie, Q. Mei, and W.-D. Han, "MicroRNAs: novel immunotherapeutic targets in colorectal carcinoma," World Journal of Gastroenterology, vol. 22, no. 23, pp. 5317-5331, 2016.

[332] M. K. Boisen, C. Dehlendorff, D. Linnemann et al., "MicroRNA expression in formalin-fixed paraffin-embedded cancer tissue: identifying reference MicroRNAs and variability," BMC Cancer, vol. 15, article 1024, 2015.

[333] Y. Xuan, H. Yang, L. Zhao et al., "MicroRNAs in colorectal cancer: small molecules with big functions," Cancer Letters, vol. 360, no. 2, pp. 89-105, 2015.

[334] T. El-Hefnawy, S. Raja, L. Kelly et al., "Characterization of amplifiable, circulating RNA in plasma and its potential as a tool for cancer diagnostics," Clinical Chemistry, vol. 50, no. 3 , pp. 564-573, 2004.

[335] E. E. Creemers, A. J. Tijsen, and Y. M. Pinto, "Circulating MicroRNAs: novel biomarkers and extracellular communicators in cardiovascular disease?" Circulation Research, vol. 110, no. 3, pp. 483-495, 2012.

[336] L. Pigati, S. C. S. Yaddanapudi, R. Iyengar et al., "Selective release of MicroRNA species from normal and malignant mammary epithelial cells," PLoS ONE, vol. 5, no. 10, Article ID e13515, 2010.

[337] T. S. Chen, R. C. Lai, M. M. Lee, A. B. H. Choo, C. N. Lee, and S. K. Lim, "Mesenchymal stem cell secretes microparticles enriched in pre-microRNAs," Nucleic Acids Research, vol. 38, no. 1, pp. 215-224, 2010.

[338] S. Kubota, M. Chiba, M. Watanabe, M. Sakamoto, and N. Watanabe, "Secretion of small/microRNAs including miR-638 into extracellular spaces by sphingomyelin phosphodiesterase 3," Oncology Reports, vol. 33, no. 1, pp. 67-73, 2015.

[339] G.-H. Liu, Z.-G. Zhou, R. Chen et al., "Serum miR-21 and miR$92 \mathrm{a}$ as biomarkers in the diagnosis and prognosis of colorectal cancer," Tumor Biology, vol. 34, no. 4, pp. 2175-2181, 2013.

[340] C. W. Wu, S. S. M. Ng, Y. J. Dong et al., "Detection of miR-92a and miR-21 in stool samples as potential screening biomarkers for colorectal cancer and polyps," Gut, vol. 61, no. 5, pp. 739-745, 2012.

[341] Z. Kanaan, S. N. Rai, M. R. Eichenberger et al., "Plasma MiR21: a potential diagnostic marker of colorectal cancer," Annals of Surgery, vol. 256, no. 3, pp. 544-551, 2012.
[342] S. T. Aherne, S. F. Madden, D. J. Hughes et al., "Circulating miRNAs miR-34a and miR-150 associated with colorectal cancer progression," BMC Cancer, vol. 15, article 329, 2015.

[343] H. Imaoka, Y. Toiyama, H. Fujikawa et al., "Circulating microRNA-1290 as a novel diagnostic and prognostic biomarker in human colorectal cancer," Annals of Oncology, vol. 27, no. 10, pp. 1879-1886, 2016.

[344] Z. Kanaan, H. Roberts, M. R. Eichenberger et al., "A plasma MicroRNA panel for detection of colorectal adenomas: a step toward more precise screening for colorectal cancer," Annals of Surgery, vol. 258, no. 3, pp. 400-408, 2013.

[345] C. W. Wu, S. C. Ng, Y. Dong et al., "Identification of microrna$135 \mathrm{~b}$ in stool as a potential noninvasive biomarker for colorectal cancer and adenoma," Clinical Cancer Research, vol. 20, no. 11, pp. 2994-3002, 2014.

[346] Y. Koga, N. Yamazaki, Y. Yamamoto et al., "Fecal miR-106a is a useful marker for colorectal cancer patients with falsenegative results in immunochemical fecal occult blood test," Cancer Epidemiology Biomarkers and Prevention, vol. 22, no. 10, pp. 1844-1852, 2013.

[347] F. E. Ahmed, "miRNA as markers for the diagnostic screening of colon cancer," Expert Review of Anticancer Therapy, vol. 14, no. 4, pp. 463-485, 2014.

[348] F. Simmer, S. Venderbosch, J. R. Dijkstra et al., "MicroRNA143 is a putative predictive factor for the response to fluoropyrimidine-based chemotherapy in patients with metastatic colorectal cancer," Oncotarget, vol. 6, no. 26, pp. 22996-23007, 2015.

[349] L. Pérez-Carbonell, F. A. Sinicrope, S. R. Alberts et al., "MiR320 e is a novel prognostic biomarker in colorectal cancer," British Journal of Cancer, vol. 113, no. 1, pp. 83-90, 2015.

[350] H.-C. Lee, J. G. Kim, Y. S. Chae et al., "Prognostic impact of microRNA-related gene polymorphisms on survival of patients with colorectal cancer," Journal of Cancer Research and Clinical Oncology, vol. 136, no. 7, pp. 1073-1078, 2010.

[351] F. Graziano, E. Canestrari, F. Loupakis et al., "Genetic modulation of the Let-7 microRNA binding to KRAS 3 '-untranslated region and survival of metastatic colorectal cancer patients treated with salvage cetuximab-irinotecan," Pharmacogenomics Journal, vol. 10, no. 5, pp. 458-464, 2010.

[352] T. F. Hansen, R. D. P. Christensen, R. F. Andersen, F. B. Sørensen, A. Johnsson, and A. Jakobsen, "MicroRNA-126 and epidermal growth factor-like domain 7-an angiogenic couple of importance in metastatic colorectal cancer. Results from the Nordic ACT trial," British Journal of Cancer, vol. 109, no. 5, pp. 1243-1251, 2013.

[353] W. Ma, J. Yu, X. Qi et al., "Radiation-induced microRNA622 causes radioresistance in colorectal cancer cells by downregulating Rb," Oncotarget, vol. 6, no. 18, pp. 15984-15994, 2015.

[354] Y. Zhang, J. Yu, H. Liu et al., "Novel epigenetic CREB-miR-630 signaling axis regulates radiosensitivity in colorectal cancer," PLoS ONE, vol. 10, no. 8, Article ID e0133870, 2015.

[355] L. Zheng, Y. Zhang, Y. Liu et al., "MiR-106b induces cell radioresistance via the PTEN/PI3K/AKT pathways and p21 in colorectal cancer," Journal of Translational Medicine, vol. 13, article 252, 2015.

[356] A. F. Christopher, R. P. Kaur, G. Kaur, A. Kaur, V. Gupta, and P. Bansal, "MicroRNA therapeutics: discovering novel targets and developing specific therapy," Perspectives in Clinical Research, vol. 7, no. 2, pp. 68-74, 2016. 
[357] J. Jung, C. Yeom, Y.-S. Choi et al., "Simultaneous inhibition of multiple oncogenic miRNAs by a multi-potent microRNA sponge," Oncotarget, vol. 6, no. 24, pp. 20370-20387, 2015.

[358] M. S. Ebert and P. A. Sharp, "MicroRNA sponges: progress and possibilities," RNA, vol. 16, no. 11, pp. 2043-2050, 2010.

[359] F. R. Kulcheski, A. P. Christoff, and R. Margis, "Circular RNAs are miRNA sponges and can be used as a new class of biomarker," Journal of Biotechnology, vol. 238, pp. 42-51, 2016.

[360] W.-Y. Choi, A. J. Giraldez, and A. F. Schier, "Target protectors reveal dampening and balancing of nodal agonist and antagonist by miR-430," Science, vol. 318, no. 5848, pp. 271-274, 2007.

[361] K. Gumireddy, D. D. Young, X. Xiong, J. B. Hogenesch, Q. Huang, and A. Deiters, "Small-molecule inhibitors of microRNA miR-21 function," Angewandte Chemie-International Edition, vol. 47, no. 39, pp. 7482-7484, 2008.

[362] V. T. Tripp and D. D. Young, "Discovery of small molecule modifiers of microRNAs for the treatment of HCV infection," Methods in Molecular Biology, vol. 1103, pp. 153-163, 2014.

[363] S. Melo, A. Villanueva, C. Moutinho et al., "Small molecule enoxacin is a cancer-specific growth inhibitor that acts by enhancing TAR RNA-binding protein 2-mediated microRNA processing," Proceedings of the National Academy of Sciences of the United States of America, vol. 108, no. 11, pp. 4394-4399, 2011.

[364] N. Valeri, C. Braconi, P. Gasparini et al., "MicroRNA-135b promotes cancer progression by acting as a downstream effector of oncogenic pathways in colon cancer," Cancer Cell, vol. 25, no. 4, pp. 469-483, 2014.

[365] N. Valeri, P. Gasparini, C. Braconi et al., "MicroRNA-21 induces resistance to 5 -fluorouracil by down-regulating human DNA MutS homolog 2 (hMSH2)," Proceedings of the National Academy of Sciences of the United States of America, vol. 107, no. 49, pp. 21098-21103, 2010.

[366] R. S. Geary, "Antisense oligonucleotide pharmacokinetics and metabolism," Expert Opinion on Drug Metabolism and Toxicology, vol. 5, no. 4, pp. 381-391, 2009.

[367] J. Krützfeldt, N. Rajewsky, R. Braich et al., "Silencing of microRNAs in vivo with 'antagomirs," Nature, vol. 438, no. 7068, pp. 685-689, 2005.

[368] E. van Rooij and S. Kauppinen, "Development of microRNA therapeutics is coming of age," EMBO Molecular Medicine, vol. 6, no. 7, pp. 851-864, 2014.

[369] S. Obad, C. O. dos Santos, A. Petri et al., "Silencing of microRNA families by seed-targeting tiny LNAs," Nature Genetics, vol. 43, no. 4, pp. 371-378, 2011.

[370] S. B. Thorsen, S. Obad, N. F. Jensen, J. Stenvang, and S. Kauppinen, "The therapeutic potential of microRNAs in cancer," Cancer Journal, vol. 18, no. 3, pp. 275-284, 2012.

[371] P. Y. Chen, L. Weinmann, D. Gaidatzis et al., "Strand-specific $5^{\prime}$-O-methylation of siRNA duplexes controls guide strand selection and targeting specificity," RNA, vol. 14, no. 2, pp. 263$274,2008$.

[372] A. G. Bader, "miR-34-a microRNA replacement therapy is headed to the clinic," Frontiers in Genetics, vol. 3, article 120, 2012.

[373] A. F. Ibrahim, U. Weirauch, M. Thomas, A. Grünweller, R. K. Hartmann, and A. Aigner, "MicroRNA replacement therapy for miR-145 and miR-33a is efficacious in a model of colon carcinoma," Cancer Research, vol. 71, no. 15, pp. 5214-5224, 2011.

[374] G. Liang, Y. Zhu, A. Jing et al., "Cationic microRNA-delivering nanocarriers for efficient treatment of colon carcinoma in xenograft model," Gene Therapy, vol. 23, no. 12, pp. 829-838, 2016.

[375] C. Wolfrum, S. Shi, K. N. Jayaprakash et al., "Mechanisms and optimization of in vivo delivery of lipophilic siRNAs," Nature Biotechnology, vol. 25, no. 10, pp. 1149-1157, 2007.

[376] E. Wagner, "Tumor-targeted delivery of anti-microRNA for cancer therapy: PHLIP is Key," Angewandte Chemie-International Edition, vol. 54, no. 20, pp. 58245826, 2015.

[377] T. Kawai and S. Akira, "The role of pattern-recognition receptors in innate immunity: update on toll-like receptors," Nature Immunology, vol. 11, no. 5, pp. 373-384, 2010.

[378] V. Hornung, M. Guenthner-Biller, C. Bourquin et al., "Sequence-specific potent induction of IFN- $\alpha$ by short interfering RNA in plasmacytoid dendritic cells through TLR7," Nature Medicine, vol. 11, no. 3, pp. 263-270, 2005. 


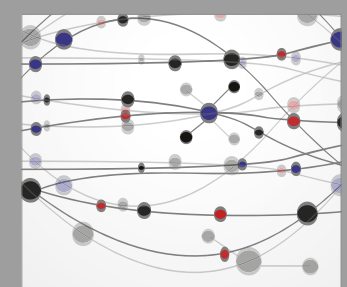

The Scientific World Journal
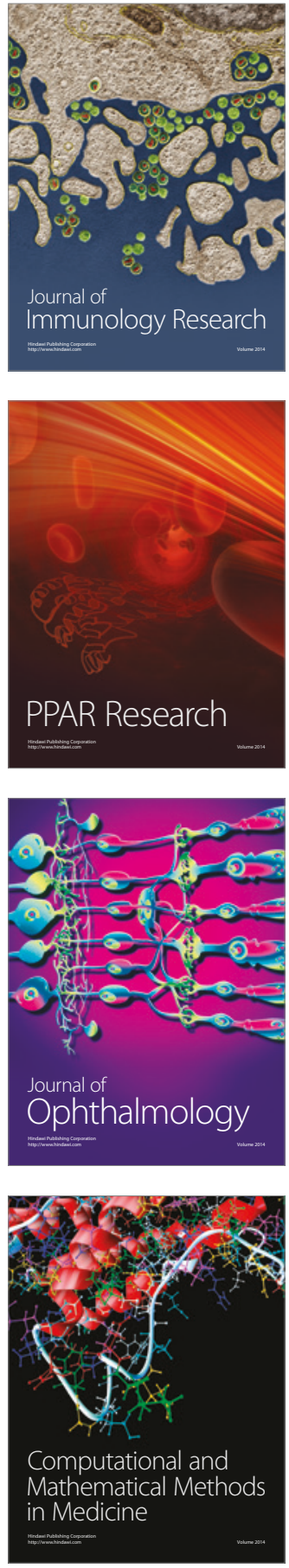

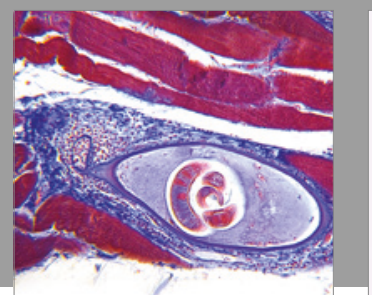

Gastroenterology Research and Practice
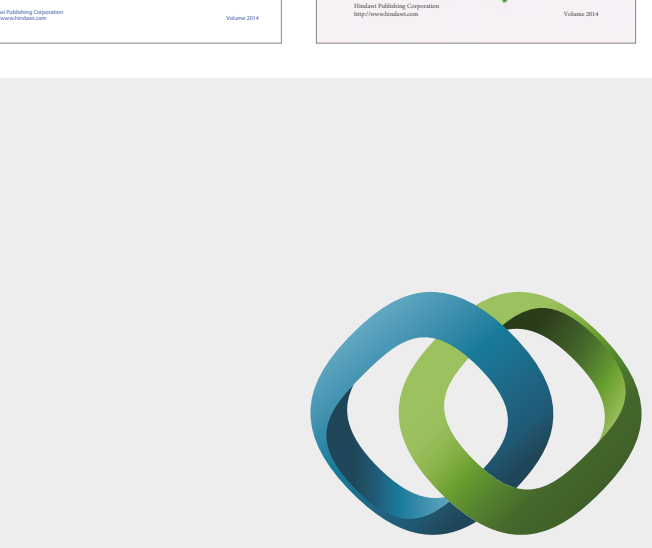

\section{Hindawi}

Submit your manuscripts at

https://www.hindawi.com
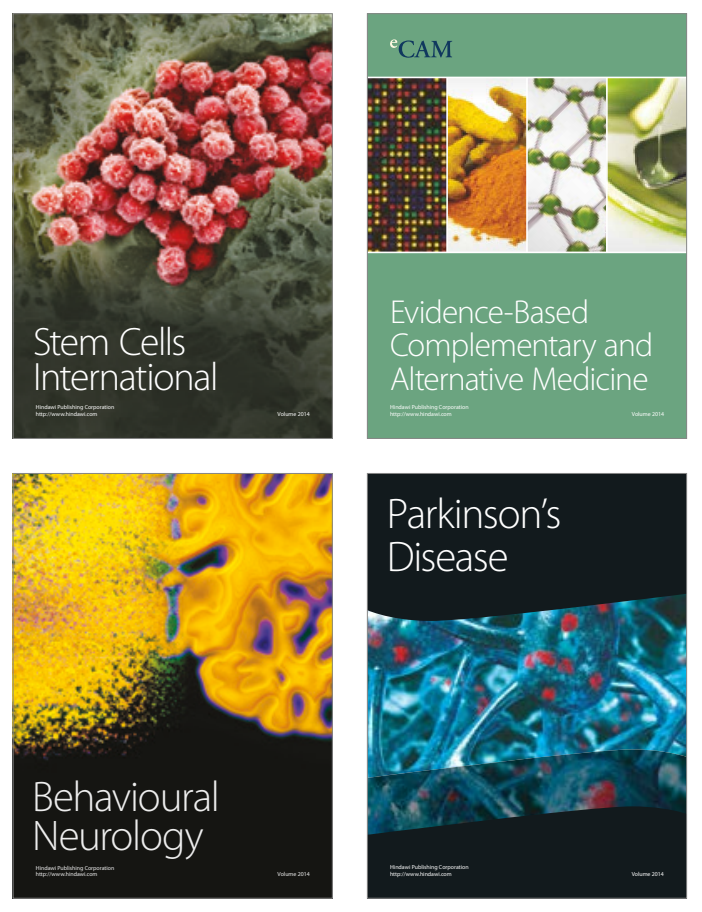
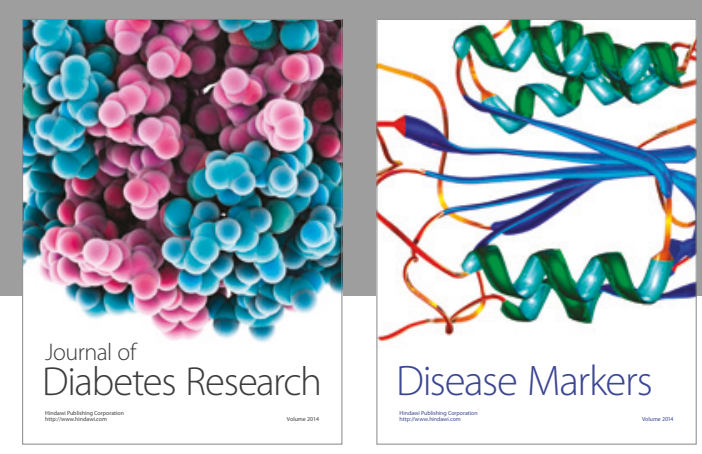

Disease Markers
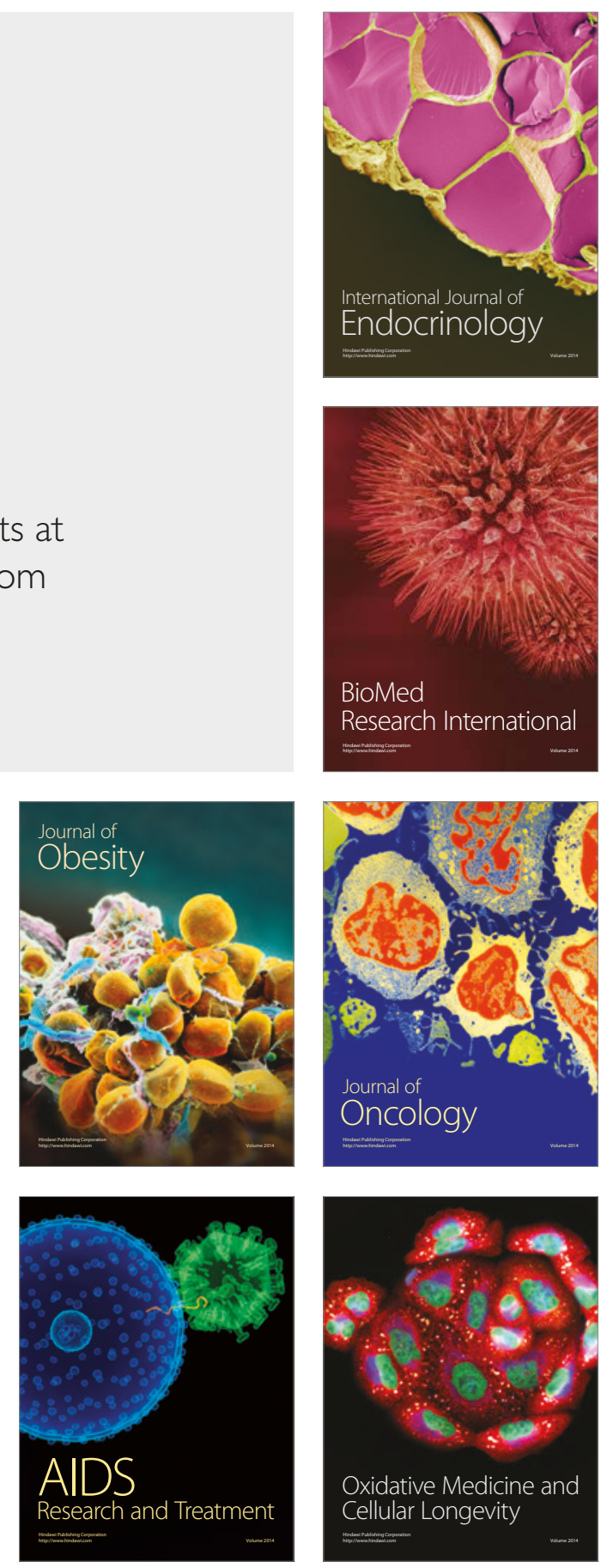